\title{
Revisiting Bisimilarity and its Modal Logic for Nondeterministic and Probabilistic Processes
}

\author{
Marco Bernardo · Rocco De Nicola · Michele Loreti
}

the date of receipt and acceptance should be inserted later

\begin{abstract}
The logic PML is a probabilistic version of Hennessy-Milner logic introduced by Larsen and Skou to characterize bisimilarity over probabilistic processes without internal nondeterminism. In this paper, two alternative interpretations of PML over nondeterministic and probabilistic processes as models are considered, and two new bisimulation-based equivalences that are in full agreement with those interpretations are provided. The new equivalences include as coarsest congruences the two bisimilarities for nondeterministic and probabilistic processes proposed by Segala and Lynch. The latter equivalences are instead known to agree with two versions of Hennessy-Milner logic extended with an additional probabilistic operator interpreted over state distributions in place of individual states. The new interpretations of PML and the corresponding new bisimilarities are thus the first ones to offer a uniform framework for reasoning on processes that are purely nondeterministic or reactive probabilistic or that mix nondeterminism and probability in an alternating/nonalternating way.
\end{abstract}

\section{Introduction}

Modal logics and behavioral equivalences play a key rôle in the specification and verification of concurrent systems. The former are useful for model checking, in that they can be employed for specifying the properties to be verified. The latter are ancillary to the former, in the sense that they enable the transformation/minimization of models to be checked while guaranteeing that specific classes of properties are preserved.

Because of this, whenever a new equivalence or a new logic is proposed, the quest starts for the associated logic or equivalence, respectively, such that two systems are behaviorally equivalent if and only if they satisfy the same modal logical formulae. The first results along this line are due to Van Benthem [4] and Hennessy and Milner [26]. The latter showed that

Marco Bernardo

Dipartimento di Scienze di Base e Fondamenti, Università di Urbino, Italy

Rocco De Nicola

IMT, Institute for Advanced Studies Lucca, Italy \& GSSI, Gran Sasso Science Institute L’Aquila, Italy

Michele Loreti

Dipartimento di Statistica, Informatica, Applicazioni, Università di Firenze, Italy 
bisimilarity over fully nondeterministic processes, each modeled as a labeled transition system (LTS) [30], is in full agreement with a very simple modal logic, now known as HML, under the image finiteness assumption (i.e., that every LTS state has finitely many outgoing transitions labeled with the same action). This logic has only four operators: true, $\cdot \wedge \cdot, \neg \cdot$, and $\langle a\rangle \cdot$, the last one being called diamond and used to describe the existence of $a$-labeled transitions. After this result, whenever any of the many quantitative variants of process description languages and process models has been introduced, other behavioral equivalences and modal logics have been defined and analogous results have been established for models handling additional features of systems, such as probability and time.

Most of the works along the lines outlined above take as starting point a behavioral equivalence and then look for the logic in agreement with it. Obviously, it is also interesting, once one has fixed a model and a logic to reason about it, to look for the "right" behavioral relation. A first work in this direction was [8]; it showed that, over finite Kripke structures (i.e., transition systems with propositions labeling states) [28], the equivalences induced by CTL* and CTL* without the next-time operator [11] are in full agreement with bisimilarity and stuttering bisimilarity, respectively. Afterwards, in [1] it was shown that, over finite probabilistic Kripke structures (i.e., Markov chains with propositions labeling states), the equivalence induced by PCTL* (an extension defined in [1] of PCTL [24]) coincides with the probabilistic bisimilarity of [33]. Recently, in [42] new probabilistic bisimilarities were introduced that fully agree with the equivalences induced by PCTL/PCTL* and their variants without the next-time operator, when interpreting these logics, as in [7], over finitely-branching nondeterministic and probabilistic Kripke structures. This is analogous to the main result of [19], which relates the equivalence induced by an action-based variant of PCTL* with the weak probabilistic bisimilarity of [36] over image-finite LTS-like models in which states have either nondeterministic transitions or probabilistic transitions.

In this paper, we concentrate on the results obtained for extended LTS models that have been developed to deal with probabilistic systems. We look for bisimilarities that are in agreement with a probabilistic variant of HML known as PML that was proposed by Larsen and Skou $[33,34]$. PML is obtained from HML by simply decorating its diamond operator with a probability bound. Formula $\langle a\rangle_{p} \phi$ is satisfied by state $s$ if an $a$-labeled transition is possible from $s$ after which a set of states satisfying $\phi$ is reached with probability at least $p$.

Larsen and Skou [33,34] introduced probabilistic bisimilarity for reactive probabilistic processes [21], which are LTS-based models where (i) every action-labeled transition reaches a probability distribution over states and (ii) the actions that label transitions departing from the same state are all different from each other. Probabilistic bisimilarity was shown to be in full agreement with PML under the minimal deviation assumption, a probabilistic variant of image finiteness. Subsequently, Desharnais et al [17] discovered that neither negation nor minimal deviation are needed to characterize probabilistic bisimilarity over a continuous-state-space generalization of reactive probabilistic processes.

Segala and Lynch [39] defined, instead, probabilistic bisimilarity over a model that also admits internal nondeterminism, i.e., the possibility for a state to have several outgoing transitions labeled with the same action. For these nondeterministic and probabilistic processes, Segala et al $[35,27]$ exhibited a logical characterization of probabilistic bisimilarity in terms of an extension of HML, in which formulae satisfaction is defined over probability distributions on states rather than over single states. The logic is obtained from HML by using infinite conjunction (so to get rid of image finiteness), by giving the diamond operator a universal interpretation (i.e., all states in the support of a distribution must satisfy the formula), and by adding a unary operator $[\cdot]_{p}$ such that $[\phi]_{p}$ is true on a state distribution if the probability assigned by the distribution to the set of states satisfying formula $\phi$ is at least $p$. 


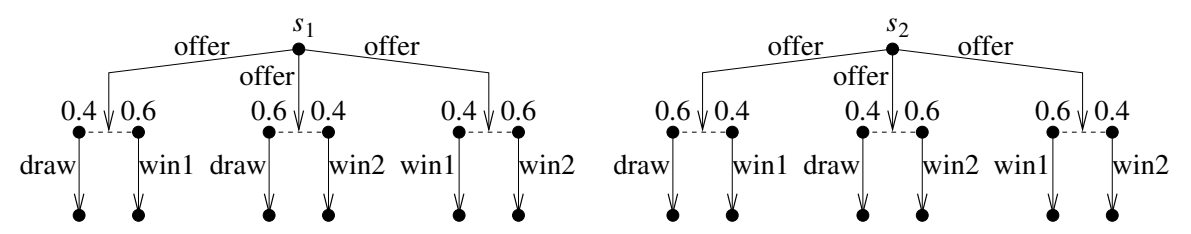

Fig. 1 Two games ensuring the same sets of probabilities of winning/drawing/losing $\left(\sim_{\mathrm{PB}, \mathrm{gbg},=}\right)$

The above-mentioned variant of HML has been reconsidered in a number of subsequent works. In [13], D'Argenio et al revised the logic in a continuous-state-space setting, by distinguishing between state formulae including the diamond operator (interpreted, as in HML, over states) and measure formulae including the new unary operator (interpreted, as by Segala et al, over state distributions). More recently, Crafa and Ranzato [12] showed an equivalent formulation of the logic that retrieves the existential interpretation of the HML diamond operator by lifting the transition relation to state distributions. Following a similar lifting, Hennessy [25] proposed an alternative logical characterization based on what he called pHML, where a binary operator $\oplus_{p}$. is added to HML (instead of the unary operator $[\cdot]_{p}$ of Segala et al) such that $\phi_{1} \oplus_{p} \phi_{2}$ asserts decomposability of a state distribution to satisfy the two subformulae.

Now, the difference between PML and the two probabilistic extensions of HML respectively defined in [35,27] and [25] is quite striking. In our view, it is thus interesting to understand whether such a difference is due to the different expressive power of the two models in [33] and [39] - i.e., the absence or the presence of internal nondeterminism - or to the way probabilistic bisimilarity was defined on those two models. Since in [35] it was shown that PML characterizes probabilistic bisimilarity over processes alternating nondeterminism and probability like those in [23] (strictly alternating processes) and in [46,36] (non-strictly alternating processes), we feel it is worth exploring alternative definitions of probabilistic bisimilarity rather than alternative models.

The aim of this paper is to show that it is possible to define new probabilistic bisimilarities for the general model of non-alternating nondeterministic and probabilistic processes [38] that are characterized by PML. Our result is somehow similar to the one established in [42], where new probabilistic bisimilarities over nondeterministic and probabilistic Kripke structures were exhibited that are characterized by PCTL/PCTL* and their variants. In both cases, the motivation for defining new probabilistic bisimilarities is the fact that the definition of Segala and Lynch [39] might be considered overdiscriminating (see also [14]) and thus differentiate processes that, according to intuition, should be identified.

To compare systems where both nondeterminism and probabilistic choices coexist, the notion of scheduler (or adversary) was used in $[38,39]$ to resolve nondeterminism. A scheduler can be viewed as an external entity that selects the next action to perform according to the current state and the past history. When a scheduler is applied to a system, a fully probabilistic model called a resolution is obtained. The basic idea is deeming equivalent two systems if for each resolution of one system (the challenger) there exists a resolution of the other (the defender) such that the two resolutions are bisimilar in the sense of [33].

Let us consider two scenarios modeling the offer to Player1 and Player2 of three differently biased dice. The game is conceived in such a way that if the outcome of a throw gives 1 or 2 then Player 1 wins, while if the outcome is 5 or 6 then Player 2 wins. In case of 3 or 4 , the result is a draw. The two scenarios are reported in Fig. 1. For instance, with the biased die associated with the leftmost branch of the first scenario, it happens that 3 or 4 (draw) will 

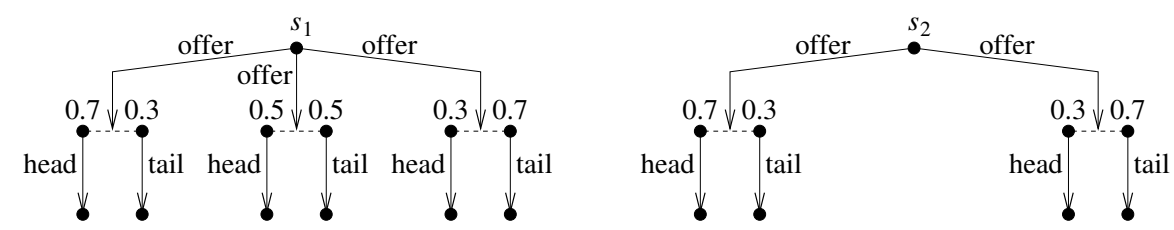

Fig. 2 Two games guaranteeing the same extremal probabilities of winning/losing $\left(\sim_{\mathrm{PB}, \mathrm{gbg}, \leq}\right)$

appear with probability 0.4 , while 1 or 2 (Player1 wins) will appear with probability 0.6. Numbers 5 and 6 will never appear (no chance for Player2 to win).

The probabilistic bisimilarity proposed in [39] differentiates the models in Fig. 1. It could be argued, however, that they should be identified because in both scenarios each player has the same sets of probabilities of winning/drawing/losing, which are all equal to $\{0.6,0.4,0\}$. To identify these systems, from a bisimulation perspective it is needed to weaken the impact of schedulers. Indeed, while in [39] the challenger and the defender must stepwise behave the same along two fully matching resolutions, here, in the same vein as [43], we admit bisimulation games with partially matching resolutions.

Other two systems differentiated (under deterministic schedulers) by the probabilistic bisimilarity of [39] are those in Fig. 2. In the first scenario, the two players are offered a choice among a fair coin and two biased ones. In the second scenario, the players can simply choose between the two biased coins of the former scenario. In both scenarios, Player1 wins with head while Player2 wins with tail. If one is interested just in extremal probabilities, it might however be argued that the two scenarios should be considered equivalent because both players have exactly the same minimal (0.3) and maximal (0.7) probability of winning/losing.

The first probabilistic bisimilarity we will introduce - denoted by $\sim_{\mathrm{PB}, \mathrm{gbg},=}=-$ identifies the two systems in Fig. 1, but distinguishes those in Fig. 2. Our second probabilistic bisimilarity - denoted by $\sim_{\mathrm{PB}, \mathrm{gbg}, \leq}-$ instead identifies both the two systems in Fig. 1 and the two systems in Fig. 2. Notably, the same identifications are induced by one of the probabilistic bisimilarities in [42]. Following [15], once the appropriate transformations (eliminating actions from transitions and labeling each state with the set of possible next-actions) are applied to get nondeterministic and probabilistic Kripke structures from the four systems in Figs. 1 and 2, we have that no PCTL* formula distinguishes the two systems in Fig. 1 and the two systems in Fig. 2 . It is, however, worth pointing out that neither $\sim_{\mathrm{PB}, \mathrm{gbg},=\text { nor }}$ $\sim_{\mathrm{PB}, \mathrm{gbg}, \leq}$ coincides with the probabilistic bisimilarities in [42].

We will show that $\sim_{\mathrm{PB}, \mathrm{gbg}, \leq}$ is precisely characterized by the original PML as defined by Larsen and Skou $[33,34]$, with the original interpretation of the diamond operator stating that state $s$ satisfies $\langle a\rangle_{p} \phi$ if $s$ has an $a$-transition that reaches with probability at least $p$ a set of states satisfying $\phi$. In contrast, $\sim_{\mathrm{PB}, \mathrm{gbg},=}$ is characterized by a variant of PML having an interval-based operator $\langle a\rangle_{\left[p_{1}, p_{2}\right]}$. instead of $\langle a\rangle_{p}$. State $s$ satisfies $\langle a\rangle_{\left[p_{1}, p_{2}\right]} \phi$ if $s$ has an $a$-transition that reaches, with probability between $p_{1}$ and $p_{2}$, a set of states satisfying $\phi$. We will refer to the interpretation of these two diamond operators as existential, because it simply requires that there exists a way of resolving internal nondeterminism that guarantees satisfaction of formula $\phi$ within a certain probability range.

For both logics, we will also provide an alternative universal interpretation of the diamond operator inspired by the interpretation of PCTL* in [7]. With this interpretation, state $s$ satisfies $\langle a\rangle_{p} \phi$ (resp. $\langle a\rangle_{\left[p_{1}, p_{2}\right]} \phi$ ) if it has an $a$-transition that enjoys the same property as before and each $a$-transition departing from $s$ enjoys that property, meaning that the formula 
is satisfied by s no matter how internal nondeterminism is resolved. Although it could be argued that in a nondeterministic and probabilistic setting such a universal interpretation is more appropriate than the existential one, we will see that both universally interpreted variants of the logic lead to the same equivalence as the one characterized by the original interpretation of the original PML. Indeed, $\sim_{\mathrm{PB}, \mathrm{gbg}, \leq}$ has also many other characterizations, and this leads us to the conviction that it is an interesting behavioral relation for nondeterministic and probabilistic processes.

This paper, which is an extended and revised version of [6], is organized as follows. In Sect. 2, we recall the necessary background about the non-alternating model of nondeterministic and probabilistic processes, together with the bisimilarities introduced in [26, 33,39] and their modal logic characterizations. The two interpretations of PML over the non-alternating model are presented in Sect. 3, while the new probabilistic bisimilarities that they characterize are exhibited in Sect. 4. In Sect. 5, we provide further motivations, variants, and results for the new probabilistic bisimilarities. In Sect. 6, we discuss the spectrum of the various behavioral and logical equivalences, and show that the new probabilistic bisimilarities contain as coarsest congruences the probabilistic bisimilarities defined in [39]. Finally, Sect. 7 draws some conclusions and hints at possible future work.

\section{Background}

In this section, we present a model for nondeterministic and probabilistic processes. Then, we recast in this general model the bisimilarity of [26] and the probabilistic bimilarity of [33], along with their HML and PML characterizations, respectively. Finally, we recall the probabilistic bisimilarities of [39] and their modal logic characterizations for both the non-alternating case and the alternating case.

\subsection{The NPLTS Model}

Processes combining nondeterminism and probability are typically described by means of extensions of the LTS model, in which every action-labeled transition goes from a source state to a probability distribution over target states rather than to a single target state. The resulting processes are essentially Markov decision processes [16] and are representative of a number of slightly different probabilistic computational models including internal nondeterminism such as, e.g., concurrent Markov chains [44], strictly alternating models [23], probabilistic automata in the sense of [38], and the denotational probabilistic models in [29] (see [41] for an overview). We formalize them as a variant of simple probabilistic automata [38] and give them the acronym NPLTS to stress the possible simultaneous presence of nondeterminism $(\mathrm{N})$ and probability $(\mathrm{P})$ in the LTS-like model.

Definition 1 A nondeterministic and probabilistic labeled transition system, NPLTS for short, is a triple $(S, A, \longrightarrow)$ where:

- $S$ is an at most countable, nonempty set of states.

- $A$ is a countable, nonempty set of transition-labeling actions.

- $\longrightarrow \subseteq S A \times \operatorname{Distr}(S)$ is a transition relation, where $\operatorname{Distr}(S)$ is the set of probability distributions over $S$. 
A transition $(s, a, \mathscr{D})$ is written $s \stackrel{a}{\longrightarrow} \mathscr{D}$. We say that $s^{\prime} \in S$ is not reachable from $s$ via that $a$-transition if $\mathscr{D}\left(s^{\prime}\right)=0$, otherwise we say that it is reachable with probability $p=\mathscr{D}\left(s^{\prime}\right)$. The reachable states form the support of $\mathscr{D}$, i.e., $\operatorname{supp}(\mathscr{D})=\left\{s^{\prime} \in S \mid \mathscr{D}\left(s^{\prime}\right)>0\right\}$. We write $s \stackrel{a}{\longrightarrow}$ to indicate that $s$ has an $a$-transition. The nondeterministic choice among all the transitions departing from $s$ can be influenced by the external environment, while the probabilistic choice of the target state for a specific transition takes place internally.

The notion of NPLTS yields a non-alternating model [38] and embeds the following restricted models:

- Fully nondeterministic processes: every transition is Dirac, i.e., it leads to a distribution that concentrates all the probability mass into a single target state.

- Fully probabilistic processes: every state has at most one outgoing transition.

- Reactive probabilistic processes: no state has two or more outgoing transitions labeled with the same action [21]. These processes include the probabilistic automata in the sense of [37].

- Alternating processes: every state that enables a non-Dirac transition enables only that transition. Similar to [46,36], these processes consist of a non-strict alternation of fully nondeterministic states and fully probabilistic states, with the addition that transitions departing from fully probabilistic states are labeled with actions.

An NPLTS can be depicted as a directed graph-like structure in which vertices represent states and action-labeled edges represent action-labeled transitions. Given a transition $s \stackrel{a}{\longrightarrow} \mathscr{D}$, the corresponding $a$-labeled edge goes from the vertex representing state $s$ to a set of vertices linked by a dashed line, each of which represents a state $s^{\prime} \in \operatorname{supp}(\mathscr{D})$ and is labeled with $\mathscr{D}\left(s^{\prime}\right)$; the label is omitted when $\mathscr{D}\left(s^{\prime}\right)=1$. Four NPLTS models have been shown in Figs. 1 and 2.

We say that an NPLTS $(S, A, \longrightarrow)$ is image finite iff for all $s \in S$ and $a \in A$ the set $\{\mathscr{D} \in$ $\operatorname{Distr}(S) \mid s \stackrel{a}{\longrightarrow} \mathscr{D}\}$ is finite. Following [33], we say that it satisfies the minimal probability assumption iff there exists $\varepsilon \in \mathbb{R}_{>0}$ such that, whenever $s \stackrel{a}{\longrightarrow} \mathscr{D}$, then for all $s^{\prime} \in S$ either $\mathscr{D}\left(s^{\prime}\right)=0$ or $\mathscr{D}\left(s^{\prime}\right) \geq \varepsilon$; this implies that $\operatorname{supp}(\mathscr{D})$ is finite because it can have at most $\lceil 1 / \varepsilon\rceil$ elements. If $\mathscr{D}\left(s^{\prime}\right)$ is a multiple of $\varepsilon$ for all $s^{\prime} \in S$, then the minimal deviation assumption is also satisfied.

Sometimes, instead of ordinary transitions, we will consider combined transitions [39], each being a convex combination of equally labeled transitions. Given an NPLTS $(S, A, \longrightarrow)$, $s \in S, a \in A$, and $\mathscr{D} \in \operatorname{Distr}(S)$, in the following we write $s \stackrel{a}{\longrightarrow} \mathscr{D}_{\mathrm{D}}$ iff there exist $n \in \mathbb{N}_{>0}$, $\left(p_{i} \in \mathbb{R}_{0,1]} \mid 1 \leq i \leq n\right)$, and $\left(s \stackrel{a}{\longrightarrow} \mathscr{D}_{i} \mid 1 \leq i \leq n\right)$ such that $\sum_{i=1}^{n} p_{i}=1$ and $\sum_{i=1}^{n} p_{i} \cdot \mathscr{D}_{i}=\mathscr{D}$.

\subsection{Bisimilarity for Fully Nondeterministic Processes}

We recast in the NPLTS model the definition of bisimilarity for fully nondeterministic processes of [26]. In this case, the target of each transition is a Dirac distribution $\delta_{s}$ for $s \in S$, i.e., $\delta_{s}(s)=1$ while $\delta_{s}\left(s^{\prime}\right)=0$ for all $s^{\prime} \in S \backslash\{s\}$.

Definition 2 Let $(S, A, \longrightarrow)$ be an NPLTS in which the target of each transition is a Dirac distribution. A relation $\mathscr{B}$ over $S$ is a bisimulation iff, whenever $\left(s_{1}, s_{2}\right) \in \mathscr{B}$, then for all actions $a \in A$ :

- For each $s_{1} \stackrel{a}{\longrightarrow} \delta_{s_{1}^{\prime}}$ there exists $s_{2} \stackrel{a}{\longrightarrow} \delta_{s_{2}^{\prime}}$ such that $\left(s_{1}^{\prime}, s_{2}^{\prime}\right) \in \mathscr{B}$.

- For each $s_{2} \stackrel{a}{\longrightarrow} \delta_{s_{2}^{\prime}}$ there exists $s_{1} \stackrel{a}{\longrightarrow} \delta_{s_{1}^{\prime}}$ such that $\left(s_{1}^{\prime}, s_{2}^{\prime}\right) \in \mathscr{B}$. 
We denote by $\sim_{B}$ the largest bisimulation.

Given an image-finite NPLTS $(S, A, \longrightarrow)$ in which the target of each transition is a Dirac distribution, the relation $\sim_{\mathrm{B}}$ is characterized by the so-called Hennessy-Milner logic (HML) [26]. The set $\mathbb{F}_{\mathrm{HML}}$ of its formulae is generated by the following grammar $(a \in A)$ :

$$
\phi::=\text { true }|\neg \phi| \phi \wedge \phi \mid\langle a\rangle \phi
$$

The semantics of HML can be defined through an interpretation function $\mathscr{M}_{\text {HML }}$ that associates with any formula in $\mathbb{F}_{\mathrm{HML}}$ the set of states satisfying the formula:

$$
\begin{aligned}
\mathscr{M}_{\mathrm{HML}}[[\text { true }]] & =S \\
\mathscr{M}_{\mathrm{HML}}[[\neg \phi]] & =S \backslash \mathscr{M}_{\mathrm{HML}}[[\phi]] \\
\mathscr{M}_{\mathrm{HML}}\left[\left[\phi_{1} \wedge \phi_{2}\right]\right] & =\mathscr{M}_{\mathrm{HML}}\left[\left[\phi_{1}\right]\right] \cap \mathscr{M}_{\mathrm{HML}}\left[\left[\phi_{2}\right]\right] \\
\mathscr{M}_{\mathrm{HML}}[[\langle a\rangle \phi]] & =\left\{s \in S \mid \exists s^{\prime} \in \mathscr{M}_{\mathrm{HML}}[[\phi]] . s \stackrel{a}{\longrightarrow} \delta_{s^{\prime}}\right\}
\end{aligned}
$$

\subsection{Bisimilarity for Reactive Probabilistic Processes}

We recast in the NPLTS model also the definition of probabilistic bisimilarity for reactive probabilistic processes of [33]. In the following, we let $\mathscr{D}\left(S^{\prime}\right)=\sum_{s^{\prime} \in S^{\prime}} \mathscr{D}\left(s^{\prime}\right)$ for $\mathscr{D} \in \operatorname{Distr}(S)$ and $S^{\prime} \subseteq S$.

Definition 3 Let $(S, A, \longrightarrow)$ be an NPLTS in which the transitions of each state have different labels. An equivalence relation $\mathscr{B}$ over $S$ is a probabilistic bisimulation iff, whenever $\left(s_{1}, s_{2}\right) \in \mathscr{B}$, then for all actions $a \in A$ and for all equivalence classes $C \in S / \mathscr{B}$ it holds that the existence of $s_{1} \stackrel{a}{\longrightarrow} \mathscr{D}_{1}$ implies the existence of $s_{2} \stackrel{a}{\longrightarrow} \mathscr{D}_{2}$ and $\mathscr{D}_{1}(C)=\mathscr{D}_{2}(C)$. We denote by $\sim_{\mathrm{PB}}$ the largest probabilistic bisimulation.

Given an NPLTS $(S, A, \longrightarrow)$ satisfying the minimal deviation assumption in which the transitions of each state have different labels, the relation $\sim_{\mathrm{PB}}$ is characterized by PML [33, 34]. The set $\mathbb{F}_{\mathrm{PML}}$ of its formulae is generated by the following grammar $\left(a \in A, p \in \mathbb{R}_{[0,1]}\right)$ :

$$
\phi::=\text { true }|\neg \phi| \phi \wedge \phi \mid\langle a\rangle_{p} \phi
$$

The semantics of PML can be defined through an interpretation function $\mathscr{M}_{\text {PML }}$ that differs from $\mathscr{M}_{\mathrm{HML}}$ only for the last clause, which becomes:

$$
\mathscr{M}_{\mathrm{PML}}\left[\left[\langle a\rangle_{p} \phi\right]\right]=\left\{s \in S \mid \exists \mathscr{D} \in \operatorname{Distr}(S) . s \stackrel{a}{\longrightarrow} \mathscr{D} \wedge \mathscr{D}\left(\mathscr{M}_{\mathrm{PML}}[[\phi]]\right) \geq p\right\}
$$

Note that, in this reactive setting, if an $a$-labeled transition exists that goes from $s$ to $\mathscr{D}$, then it is the only $a$-labeled transition departing from $s$, and hence $\mathscr{D}$ is unique.

In [17], it was subsequently shown - by working in a continuous-state setting - that probabilistic bisimilarity for reactive probabilistic processes can be characterized by PML without negation, and that the existence of neither a minimal deviation nor a minimal probability needs to be assumed to achieve the characterization result.

\subsection{Bisimilarity for Non-Alternating and Alternating Processes}

We now recall for non-alternating processes (i.e., NPLTS models in their full generality) two probabilistic bisimulation equivalences defined in [39]. Both of them check whether the probabilities of all classes of equivalent states - i.e., the class distributions - reached by the two transitions considered in the bisimulation game are equal.

The first equivalence relies on deterministic schedulers for resolving nondeterminism. This means that, when responding to an $a$-transition of the challenger, the defender can only select a single $a$-transition (if any). 
Definition 4 Let $(S, A, \longrightarrow)$ be an NPLTS. An equivalence relation $\mathscr{B}$ over $S$ is a classdistribution probabilistic bisimulation iff, whenever $\left(s_{1}, s_{2}\right) \in \mathscr{B}$, then for all actions $a \in A$ it holds that for each $s_{1} \stackrel{a}{\longrightarrow} \mathscr{D}_{1}$ there exists $s_{2} \stackrel{a}{\longrightarrow} \mathscr{D}_{2}$ such that, for all equivalence classes $C \in S / \mathscr{B}, \mathscr{D}_{1}(C)=\mathscr{D}_{2}(C)$. We denote by $\sim_{\mathrm{PB} \text {,dis }}$ the largest class-distribution probabilistic bisimulation.

While in Def. 3 the quantification over $C \in S / \mathscr{B}$ can be placed before or after the transition matching because $s_{1}$ and $s_{2}$ can have at most one outgoing $a$-transition each, in Def. 4 it is important for the quantification to be after the transition matching.

The second equivalence is coarser than the first one because it relies instead on randomized schedulers. This means that, when responding to an $a$-transition of the challenger, the defender can select a convex combination of $a$-transitions (if any). In the following, the acronym ct stands for "based on combined transitions".

Definition 5 Let $(S, A, \longrightarrow)$ be an NPLTS. An equivalence relation $\mathscr{B}$ over $S$ is a classdistribution ct-probabilistic bisimulation iff, whenever $\left(s_{1}, s_{2}\right) \in \mathscr{B}$, then for all actions $a \in A$ it holds that for each $s_{1} \stackrel{a}{\longrightarrow} \mathscr{D}_{1}$ there exists $s_{2} \stackrel{a}{\longrightarrow}_{\mathrm{c}} \mathscr{D}_{2}$ such that, for all equivalence classes $C \in S / \mathscr{B}, \mathscr{D}_{1}(C)=\mathscr{D}_{2}(C)$. We denote by $\sim_{\mathrm{PB} \text {,dis }}^{\mathrm{ct}}$ the largest class-distribution ct-probabilistic bisimulation.

In order to obtain a modal logic characterization for $\sim_{\mathrm{PB}, \text { dis }}$ and $\sim_{\mathrm{PB}, \mathrm{dis}}^{\mathrm{ct}}$, in $[35,27]$ an extension of HML much richer than PML was defined. The main differences are that (i) formulae are interpreted over probability distribution on states rather than over single states and (ii) the modal operator $\langle a\rangle_{p}$. is split into the original modal operator $\langle a\rangle \cdot$ of HML and an additional unary operator $[\cdot]_{p}$. State distribution $\mathscr{D}$ satisfies $[\phi]_{p}$ if $\mathscr{D}$ assigns to the set of states satisfying $\phi$ a probability that is at least $p$.

In [25], the same equivalences (lifted to state distributions) were differently characterized by adding to HML a binary operator $\cdot \oplus_{p} \cdot$, where $\phi_{1} \oplus_{p} \phi_{2}$ asserts decomposability of a state distribution to satisfy the two subformulae.

For alternating processes, i.e., NPLTS models in which every state that enables a nonDirac transition enables only that transition, the following properties hold:

- $\sim_{\mathrm{PB} \text {,dis }}$ and $\sim_{\mathrm{PB} \text {,dis }}^{\mathrm{ct}}$ collapse into a single equivalence that coincides with those defined in $[23,36]$ for alternating processes, as shown in [40].

$-\sim_{\mathrm{PB}, \mathrm{dis}}$ is again characterized by the original PML, as shown in [35].

\section{Interpreting PML over NPLTS Models}

The modal logic PML was originally interpreted in $[33,34]$ on reactive probabilistic processes and then in [35] on alternating processes. The same interpretation can be applied to general NPLTS models by establishing that state $s$ satisfies formula $\langle a\rangle_{p} \phi$ iff there exists a resolution of the internal nondeterminism on action $a$ such that $s$ can perform an $a$-transition after which it reaches with probability at least $p$ a set of states that satisfy $\phi$. This existential interpretation only provides a weak guarantee of fulfilling properties, as it depends on how internal nondeterminism is resolved.

A different interpretation can be adopted by following [7]: $s$ satisfies $\langle a\rangle_{p} \phi$ iff, for each resolution of the internal nondeterminism on action $a, s$ can perform an $a$-transition after which it reaches with probability at least $p$ a set of states that satisfy $\phi$. The resulting universal interpretation provides a strong guarantee of fulfilling properties because, no matter how internal nondeterminism is resolved, a certain behavior is ensured. 
We denote by $\mathrm{PML}_{\exists, \geq}$ and $\mathrm{PML}_{\forall, \geq}$ the logics resulting from the two different interpretations of the diamond operator, which we formalize as follows:

$$
\begin{aligned}
& \mathscr{M}_{\mathrm{PML}_{\exists, \geq}} \llbracket\langle a\rangle_{p} \phi \rrbracket=\left\{s \in S \mid \exists \mathscr{D} \cdot s \stackrel{a}{\longrightarrow} \mathscr{D} \wedge \mathscr{D}\left(\mathscr{M}_{\mathrm{PML}_{\exists, \geq}}[\phi \phi]\right) \geq p\right\} \\
& \mathscr{M}_{\mathrm{PML}_{\forall, \geq}} \llbracket\langle a\rangle_{p} \phi \rrbracket=\left\{s \in S \mid s \stackrel{a}{\longrightarrow} \wedge \forall \mathscr{D} \cdot s \stackrel{a}{\longrightarrow} \mathscr{D} \Longrightarrow \mathscr{D}\left(\mathscr{M}_{\mathrm{PML}_{\forall, \geq}}[[\phi]) \geq p\right\}\right.
\end{aligned}
$$

We denote by $\mathrm{PML}_{\exists, \leq}$ and $\mathrm{PML}_{\forall, \leq}$ the two variants in which the probability value $p$ decorating the diamond operator is intended as an upper bound rather than a lower bound.

Finally, we denote by $\mathrm{PML}_{\exists, \mathrm{I}}$ and $\mathrm{PML}_{\forall, \mathrm{I}}$ two further variants generalizing the previous four logics, in which the probability value $p$ is replaced by a probability interval $\left[p_{1}, p_{2}\right]-$ where $p_{1}, p_{2} \in \mathbb{R}_{[0,1]}$ are such that $p_{1} \leq p_{2}$ - and the resulting diamond operator is interpreted as follows:

$$
\begin{aligned}
& \mathscr{M}_{\mathrm{PML}_{\exists, \mathrm{I}}}\left[\left\langle\langle a\rangle_{\left[p_{1}, p_{2}\right]} \phi\right]\right]=\left\{s \in S \mid \exists \mathscr{D} . s \stackrel{a}{\longrightarrow} \mathscr{D} \wedge p_{1} \leq \mathscr{D}\left(\mathscr{M}_{\mathrm{PML}_{\exists, \mathrm{I}}}[[\phi]]\right) \leq p_{2}\right\} \\
& \left.\mathscr{M}_{\mathrm{PML}_{\forall, \mathrm{I}}}\left[\langle a\rangle_{\left[p_{1}, p_{2}\right]} \phi\right]\right]=\left\{s \in S \mid s \stackrel{a}{\longrightarrow} \wedge \forall \mathscr{D} \cdot s \stackrel{a}{\longrightarrow} \mathscr{D} \Longrightarrow p_{1} \leq \mathscr{D}\left(\mathscr{M}_{\mathrm{PML}_{\forall, \mathrm{I}}}[[\phi]]\right) \leq p_{2}\right\}
\end{aligned}
$$

Note that $\langle a\rangle_{p} \phi$ can be encoded as $\langle a\rangle_{[p, 1]} \phi$ when $p$ is a lower bound and as $\langle a\rangle_{[0, p]} \phi$ when $p$ is an upper bound.

In the following, if $\mathrm{L}$ is one of the above six variants of PML, then we denote by $\mathscr{F}_{\mathrm{L}}(s)$ the set of formulae in $\mathbb{F}_{\mathrm{L}}$ satisfied by state $s$ and we let $s_{1} \sim_{\mathrm{L}} s_{2}$ iff $\mathscr{F}_{\mathrm{L}}\left(s_{1}\right)=\mathscr{F}_{\mathrm{L}}\left(s_{2}\right)$.

Interestingly enough, in Sect. 6 we will see that the equivalences induced by the three universally interpreted variants are the same, and coincide with the equivalences induced by the two existentially interpreted variants with probabilistic bound; in contrast, the equivalence induced by $\mathrm{PML}_{\exists, \mathrm{I}}$ is finer then the one induced by the other five variants. We will also see that the six equivalences are different by those induced by the logics of $[35,27,25]$.

\section{Group-by-Group Probabilistic Bisimilarities and PML}

In this section, we introduce the probabilistic bisimilarities for NPLTS models that are characterized by PML as interpreted in the previous section. Before presenting their definition and the related results, we highlight the differences with respect to $\sim_{\mathrm{PB}, \mathrm{dis}}$.

The first difference is that, instead of comparing the probability distributions over all classes of equivalent states reached by the transitions considered in the bisimulation game, the new equivalences focus on a single equivalence class at a time. This is similar to the approach followed in [43] in the setting of approximate probabilistic relations. For an action $a$, the probability distribution over all classes of equivalent states reached by an $a$-transition of the challenger can now be matched by means of several (not just one) $a$-transitions of the defender, each taking care of a different class, thus working in a class-by-class fashion.

Secondly, the new equivalences take into account the probability of reaching groups of equivalence classes rather than only individual classes. This is similar to the approaches followed in $[18]$ and in $[9,13]$ to ensure transitivity of probabilistic bisimilarity over continuousstate probabilistic processes without and with internal nondeterminism, respectively. Considering groups of equivalence classes in $\sim_{\mathrm{PB} \text {,dis }}$ would make no difference, while dealing only with individual classes here would significantly weaken the discriminating power, as will be illustrated in Sect. 5.1. Due to the first two differences with respect to $\sim_{\mathrm{PB} \text {,dis }}$, we call these new equivalences group-by-group probabilistic bisimilarities.

Thirdly, the new equivalences come in several variants depending on whether, in the bisimulation game, the probabilities of reaching a certain group of classes of equivalent states are compared based on $=, \leq$, or $\geq$. Similar to the consideration of groups of classes instead of individual classes, this would make no difference in the case of $\sim_{\mathrm{PB} \text {,dis }}$. 
4.1 Defining Group-by-Group Probabilistic Bisimilarities

In the following, we let $\bigcup \mathscr{G}=\bigcup_{C \in \mathscr{G}} C$ when $\mathscr{G} \in 2^{S / \mathscr{B}}$ is a group of equivalence classes with respect to an equivalence relation $\mathscr{B}$ over $S$, and we use $\bowtie$ to denote one of the relational operators in $\{=, \leq, \geq\}$. The definition of $\sim_{\mathrm{PB}, \mathrm{gbg}, \bowtie}$ below assumes the use of deterministic schedulers, but it can be easily extended to the case of randomized schedulers by analogy with $\sim_{\mathrm{PB}, \mathrm{dis}}^{\mathrm{ct}}$, thus yielding $\sim_{\mathrm{PB}, \mathrm{gbg}, \triangleright}^{\mathrm{ct}}$ that will be studied in Sect. 6.

Definition 6 Let $(S, A, \longrightarrow)$ be an NPLTS. An equivalence relation $\mathscr{B}$ over $S$ is a $\bowtie$-groupby-group probabilistic bisimulation iff, whenever $\left(s_{1}, s_{2}\right) \in \mathscr{B}$, then for all actions $a \in A$ and for all groups of equivalence classes $\mathscr{G} \in 2^{S / \mathscr{B}}$ it holds that for each $s_{1} \stackrel{a}{\longrightarrow} \mathscr{D}_{1}$ there exists $s_{2} \stackrel{a}{\longrightarrow} \mathscr{D}_{2}$ such that $\mathscr{D}_{1}(\bigcup \mathscr{G}) \bowtie \mathscr{D}_{2}(\bigcup \mathscr{G})$. We denote by $\sim_{\text {PB.gbg. } \bowtie}$ the largest $\bowtie$-group-bygroup probabilistic bisimulation.

Note that, while in Def. 4 the quantification over $C \in S / \mathscr{B}$ is after the transition matching, in Def. 6 the quantification over $\mathscr{G} \in 2^{S / \mathscr{B}}$ is before the transition matching, thus allowing a transition of the challenger to be matched by several transitions of the defender depending on the target groups.

The relation $\sim_{\mathrm{PB}, \mathrm{gbg}}$, = identifies the two systems in Fig. 1, as witnessed by the =-groupby-group probabilistic bisimulation that pairs states enabling the same actions. For instance, the leftmost transition of $s_{1}$ is respectively matched by the central transition of $s_{2}$ when considering the group of states enabling only draw, the rightmost transition of $s_{2}$ when considering the group of states enabling only win 1 , and the leftmost transition of $s_{2}$ when considering the group of states enabling draw or win1. The relations $\sim_{\mathrm{PB}, \mathrm{gbg}, \leq}$ and $\sim_{\mathrm{PB}, \mathrm{gbg}, \geq}$ identify the two systems in Fig. 2 too. For example, under $\leq$ the central transition of $s_{1}$ is respectively matched by the leftmost transition of $s_{2}$ when considering the group of states enabling only head, the rightmost transition of $s_{2}$ when considering the group of states enabling only tail, and any of the two transitions of $s_{2}$ when considering the group of states enabling head or tail. In Sect. 6 , we will see that $\sim_{\mathrm{PB} \text {, dis }}$ is finer than $\sim_{\mathrm{PB}, \mathrm{gbg},=}$ and that the latter is finer than $\sim_{\mathrm{PB}, \mathrm{gbg}, \leq}$, which coincides with $\sim_{\mathrm{PB}, \mathrm{gbg}, \geq}$.

\subsection{Backward Compatibility}

Before moving to the modal logic characterization results, as a sanity check we show that the three group-by-group probabilistic bisimilarities and their ct-variants are backward compatible with the bisimilarity $\sim_{B}$ of [26] for fully nondeterministic processes (see Def. 2) and the probabilistic bisimilarity $\sim_{\mathrm{PB}}$ of [33] for reactive probabilistic processes (see Def. 3). Moreover, they coincide with the probabilistic bisimilarities $\sim_{\mathrm{PB} \text {,dis }}$ and $\sim_{\mathrm{PB} \text {,dis }}^{\mathrm{ct}}$ of [39] (see Defs. 4 and 5) when restricting attention to alternating processes.

Proposition 1 Let $(S, A, \longrightarrow)$ be an NPLTS in which the target of each transition is a Dirac distribution. Let $s_{1}, s_{2} \in S$ and $\bowtie \in\{=, \leq, \geq\}$. Then:

$$
s_{1} \sim \text { PB,gbg, } s_{2} \Longleftrightarrow s_{1} \sim_{\mathrm{PB}, \mathrm{gbg}, \bowtie}^{\mathrm{ct}} s_{2} \Longleftrightarrow s_{1} \sim_{\mathrm{B}} s_{2}
$$

Proof Since every transition of this specific NPLTS can reach with probability greater than 0 a single state and hence a single class of any equivalence relation - which are thus reached with probability 1 - the reflexive, symmetric, and transitive closure of a bisimulation is trivially $a \bowtie$-group-by-group (ct-)probabilistic bisimulation. 
Proposition 2 Let $(S, A, \longrightarrow)$ be an NPLTS in which the transitions of each state have different labels. Let $s_{1}, s_{2} \in S$ and $\bowtie \in\{=, \leq, \geq\}$. Then:

$$
s_{1} \sim_{\mathrm{PB}, \mathrm{gbg}, \bowtie} s_{2} \Longleftrightarrow s_{1} \sim_{\mathrm{PB}, \mathrm{gbg}, \bowtie}^{\mathrm{ct}} s_{2} \Longleftrightarrow s_{1} \sim_{\mathrm{PB}} s_{2}
$$

Proof Since every state of this specific NPLTS has at most one transition labeled with a certain action, a probabilistic bisimulation is trivially $a \bowtie$-group-by-group (ct-)probabilistic bisimulation.

Proposition 3 Let $(S, A, \longrightarrow)$ be an NPLTS in which every state that enables a non-Dirac transition enables only that transition. Let $s_{1}, s_{2} \in S$ and $\bowtie \in\{=, \leq, \geq\}$. Then:

$$
\begin{aligned}
& s_{1} \sim_{\mathrm{PB}, \mathrm{gbg}, \bowtie} s_{2} \Longleftrightarrow s_{1} \sim_{\mathrm{PB}, \mathrm{dis}} s_{2} \\
& s_{1} \sim_{\mathrm{PB}, \mathrm{gbg}, \bowtie}^{\mathrm{ct}} s_{2} \Longleftrightarrow s_{1} \sim_{\mathrm{PB}, \mathrm{dis}}^{\mathrm{ct}} s_{2}
\end{aligned}
$$

Proof Since every state of this specific NPLTS has either zero or more Dirac transitions or a single non-Dirac transition, a class-distribution (ct-)probabilistic bisimulation is trivially $a \bowtie$-group-by-group (ct-)probabilistic bisimulation.

\subsection{Logical Characterization}

The relation $\sim_{\mathrm{PB}, \mathrm{gbg},=}$ turns out to be characterized by $\mathrm{PML}_{\exists, \mathrm{I}}$ under assumptions of image finiteness and minimal probability. The proof of this result follows the same two-step pattern as the proof for the analogous result in [26] connecting $\sim_{B}$ with HML.

The first step consists of providing an alternative characterization of $\sim_{\mathrm{PB}, \mathrm{gbg},=}$ as the limit of a sequence of coarser equivalence relations $\sim_{\mathrm{PB}, \mathrm{gbg},=}^{i}, i \in \mathbb{N}$, which are inductively defined as follows for an NPLTS $(S, A, \longrightarrow)$ :

$-\sim_{\mathrm{PB}, \mathrm{gbg},=}^{0}=S \times S$.

- $\sim_{\mathrm{PB}, \mathrm{gbg},=}^{i+1}$ is the set of all pairs $\left(s_{1}, s_{2}\right) \in \sim_{\mathrm{PB}, \mathrm{gbg},=}^{i}$ such that for all actions $a \in A$ and groups of equivalence classes $\mathscr{G} \in 2^{S / \sim_{\mathrm{PB}, \mathrm{gbg},=}^{i}}$ it holds that for each $s_{1} \stackrel{a}{\longrightarrow} \mathscr{D}_{1}$ there exists $s_{2} \stackrel{a}{\longrightarrow} \mathscr{D}_{2}$ such that $\mathscr{D}_{1}(\bigcup \mathscr{G})=\mathscr{D}_{2}(\bigcup \mathscr{G})$.

Each equivalence relation $\sim_{\mathrm{PB}, \mathrm{gbg},=}^{i}$ identifies those states that cannot be distinguished through the bisimulation game within $i$ consecutive transitions. The following lemma guarantees that two states of an image-finite NPLTS are equivalent according to $\sim_{\mathrm{PB}, \mathrm{gbg},=}$ iff they are equivalent according to all the relations $\sim_{\mathrm{PB}, \mathrm{gbg},=}^{i}$. Its proof is a group-based adaptation of the proof developed in [2] for a similar result concerned with $\sim_{\mathrm{PB} \text {,dis }}$.

Lemma 1 Let $(S, A, \longrightarrow)$ be an image-finite NPLTS. Then:

$$
\sim \sim_{\mathrm{PB}, \mathrm{gbg},=}=\bigcap_{i \in \mathbb{N}} \sim_{\mathrm{PB}, \mathrm{gbg},=}^{i}
$$

Proof For the sake of convenience, we denote by $\sim^{\prime}$ the relation $\bigcap_{i \in \mathbb{N}} \sim_{\mathrm{PB}, \mathrm{gbg},=}^{i}$.

Firstly, we observe what follows:

- $\sim^{\prime}$ is an equivalence relation because so is $\sim_{\mathrm{PB}, \mathrm{gbg},=}^{i}$ for all $i \in \mathbb{N}$.

- Given $C \in S / \sim^{\prime}$ and $i \in \mathbb{N}$, there exists a unique element $C_{i}$ in $S / \sim_{\mathrm{PB}, \mathrm{gbg},=}^{i}$ such that $C_{i} \supseteq C$, and hence $C=\bigcap_{i \in \mathbb{N}} C_{i}$ with $C_{i_{1}} \supseteq C_{i_{2}}$ for $i_{1} \leq i_{2}$.

- As a consequence, given $\mathscr{G} \in 2^{S / \sim^{\prime}}$ and $i \in \mathbb{N}$, there exists a unique element $\mathscr{G}_{i}$ in $2^{S / \sim_{\mathrm{PB}, \mathrm{gbg},=}^{i}}$ such that every class in $\mathscr{G}_{i}$ contains some class in $\mathscr{G}$, and hence $\bigcup \mathscr{G}=$ $\bigcap_{i \in \mathbb{N}}\left(\bigcup \mathscr{G}_{i}\right)$ with $\bigcup \mathscr{G}_{i_{1}} \supseteq \bigcup \mathscr{G}_{i_{2}}$ for $i_{1} \leq i_{2}$. 
- Moreover, if $s \stackrel{a}{\longrightarrow} \mathscr{D}$, then $\mathscr{D}(\bigcup \mathscr{G})=\inf _{i \in \mathbb{N}} \mathscr{D}\left(\bigcup \mathscr{G}_{i}\right)$. In fact, observing that for all $i \in \mathbb{N}$ it holds that $\mathscr{D}\left(\bigcup \mathscr{G}_{i}\right) \geq \mathscr{D}(\bigcup \mathscr{G})$ because $\bigcup \mathscr{G}_{i} \supseteq \bigcup \mathscr{G}$, if we let $p=\inf _{i \in \mathbb{N}} \mathscr{D}\left(\bigcup \mathscr{G}_{i}\right)$, then $p \geq \mathscr{D}(\bigcup \mathscr{G})$ because $\mathscr{D}(\bigcup \mathscr{G})$ is a lower bound of the sequence $\left(\mathscr{D}\left(\bigcup \mathscr{G}_{i}\right)\right)_{i \in \mathbb{N}}$ and $p$ is the greatest lower bound of that sequence. Suppose that $p>\mathscr{D}(\bigcup \mathscr{G})$ and let $\delta=p-\mathscr{D}(\bigcup \mathscr{G})$. Since $\delta>0$ and the summation $\mathscr{D}(S \backslash \bigcup \mathscr{G})$ satisfies $\sum_{s \in S \backslash \cup \mathscr{G}} \mathscr{D}(s) \leq 1$ and hence converges, there exists a finite subset $X$ of $S \backslash \bigcup \mathscr{G}$ such that the rest $\mathscr{D}((S \backslash$ $\cup \mathscr{G}) \backslash X)$ of the previously considered summation satisfies $\sum_{s \in(S \backslash \cup \mathscr{G}) \backslash X} \mathscr{D}(s)<\delta$. Let $Y=(S \backslash \bigcup \mathscr{G}) \backslash X$. For all $i \in \mathbb{N}$, it holds that:

$$
\bigcup \mathscr{G}_{i}=\bigcup \mathscr{G} \cup\left(Y \cap \bigcup \mathscr{G}_{i}\right) \cup\left(X \cap \bigcup \mathscr{G}_{i}\right)
$$

where the three sets on the right-hand side are pairwise disjoint and hence:

$$
\begin{aligned}
\mathscr{D}\left(\bigcup \mathscr{G}_{i}\right) & =\mathscr{D}(\bigcup \mathscr{G})+\mathscr{D}\left(Y \cap \bigcup \mathscr{G}_{i}\right)+\mathscr{D}\left(X \cap \bigcup \mathscr{G}_{i}\right) \\
& \leq \mathscr{D}(\bigcup \mathscr{G})+\mathscr{D}(Y)+\mathscr{D}\left(X \cap \bigcup \mathscr{G}_{i}\right) \\
& <\mathscr{D}(\bigcup \mathscr{G})+\delta+\mathscr{D}\left(X \cap \bigcup \mathscr{G}_{i}\right) \\
& =p+\mathscr{D}\left(X \cap \bigcup \mathscr{G}_{i}\right)
\end{aligned}
$$

From the inequality above and $\mathscr{D}\left(\bigcup \mathscr{G}_{i}\right) \geq p$, we derive that:

$$
\mathscr{D}\left(X \cap \bigcup \mathscr{G}_{i}\right)>\mathscr{D}\left(\bigcup \mathscr{G}_{i}\right)-p \geq 0
$$

and hence $X \cap \bigcup \mathscr{G}_{i} \neq \emptyset$ for all $i \in \mathbb{N}$. As a consequence, $X \cap \bigcup \mathscr{G} \neq \emptyset$ because $X$ is finite and $\bigcup \mathscr{G}_{0} \supseteq \bigcup \mathscr{G}_{1} \supseteq \ldots$... This contradicts the fact that $X$ is a subset of $S \backslash \bigcup \mathscr{G}$. Therefore, it must be $p=\mathscr{D}(\bigcup \mathscr{G})$.

Secondly, it holds that $\sim^{\prime} \supseteq \sim_{\mathrm{PB}, \mathrm{gbg},=}$ because $\sim_{\mathrm{PB}, \mathrm{gbg},=}^{i} \supseteq \sim_{\mathrm{PB}, \mathrm{gbg},=}$ for all $i \in \mathbb{N}$ as we now show by proceeding by induction on $i$ :

- If $i=0$, then $\sim_{\mathrm{PB}, \mathrm{gbg},=}^{i}=S \times S \supseteq \sim_{\mathrm{PB}, \mathrm{gbg},=}$.

- Let $i$ be an element of $\mathbb{N}$ for which the result holds and consider $i+1$. If $s_{1}, s_{2} \in S$ satisfy $s_{1} \sim \mathrm{PB}, \mathrm{gbg},=s_{2}$, then:

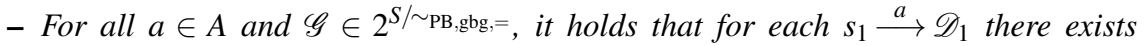
$s_{2} \stackrel{a}{\longrightarrow} \mathscr{D}_{2}$ such that $\mathscr{D}_{1}(\bigcup \mathscr{G})=\mathscr{D}_{2}(\bigcup \mathscr{G})$.

- $s_{1} \sim_{\mathrm{PB}, \mathrm{gbg},=}^{i} s_{2}$ because $\sim_{\mathrm{PB}, \mathrm{gbg},=}^{i} \supseteq \sim_{\mathrm{PB}, \mathrm{gbg},=}$ by the induction hypothesis.

Since every equivalence class of $\sim_{\mathrm{PB}, \mathrm{gbg},=}^{i}$ is equal to the union of some equivalence classes of $\sim_{\mathrm{PB}, \mathrm{gbg}},=$ and hence the union of equivalence classes in every group $\mathscr{G}^{\prime}$ of $\sim_{\mathrm{PB}, \mathrm{gbg},=}^{i}$ is equal to the union of the equivalence classes in some group $\mathscr{G}$ of $\sim_{\mathrm{PB}, \mathrm{gbg},=}$, we derive that for all $a \in A$ and $\mathscr{G}^{\prime} \in 2^{S / \sim_{\mathrm{PB}, \mathrm{gbg},=}^{i}}$ it holds that for each $s_{1} \stackrel{a}{\longrightarrow} \mathscr{D}_{1}$ there exists $s_{2} \stackrel{a}{\longrightarrow} \mathscr{D}_{2}$ such that:

This means that $s_{1} \sim{ }_{\mathrm{PB}, \mathrm{gbg},=}^{i+1} s_{2}$.

$$
\mathscr{D}_{1}\left(\bigcup \mathscr{G}^{\prime}\right)=\mathscr{D}_{1}(\bigcup \mathscr{G})=\mathscr{D}_{2}(\bigcup \mathscr{G})=\mathscr{D}_{2}\left(\bigcup \mathscr{G}^{\prime}\right)
$$

Thirdly, we prove that $\sim^{\prime} \subseteq \sim_{\mathrm{PB}, \mathrm{gbg},=}$ by showing that $\sim^{\prime}$ is $a=$-group-by-group probabilistic bisimulation. Suppose that $s_{1}, s_{2} \in S$ satisfy $s_{1} \sim^{\prime} s_{2}$ and, given $a \in A$ and $\mathscr{G} \in 2^{S / \sim^{\prime}}$, assume that $s_{1} \stackrel{a}{\longrightarrow} \mathscr{D}_{1}$. Then $\mathscr{D}_{1}(\bigcup \mathscr{G})=\inf _{i \in \mathbb{N}} \mathscr{D}_{1}\left(\cup \mathscr{G}_{i}\right)$ where each $\mathscr{G}_{i}$ is the unique el-

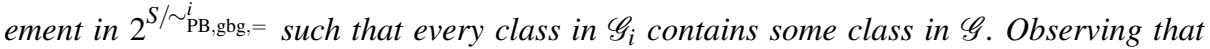
$\sim_{\mathrm{PB}, \mathrm{gbg},=}^{0}$ induces a single equivalence class equal to $S$ and hence $\mathscr{D}_{1}\left(\bigcup \mathscr{G}_{0}\right)=\mathscr{D}_{1}(S)=1$, from $s_{1} \sim^{\prime} s_{2}$ and $s_{1} \stackrel{a}{\longrightarrow} \mathscr{D}_{1}$ it follows that for all $i \in \mathbb{N}_{\geq 1}$ there exists $s_{2} \stackrel{a}{\longrightarrow} \mathscr{D}_{2, i}$ such that $\mathscr{D}_{1}\left(\bigcup \mathscr{G}_{i-1}\right)=\mathscr{D}_{2, i}\left(\bigcup \mathscr{G}_{i-1}\right)$.

Since the NPLTS is image finite, the set $\left\{\mathscr{D}_{2, i} \mid i \in \mathbb{N}_{>1}\right\}$ is finite and we enumerate it as $\left\{\Delta_{2}^{1}, \ldots, \Delta_{2}^{k}\right\}$. For each $j \in\{1, \ldots, k\}$, we also let $I_{j}$ be the set of indexes $i \in \mathbb{N}_{\geq 1}$ such that $\mathscr{D}_{1}\left(\bigcup \mathscr{G}_{i-1}\right)=\Delta_{2}^{j}\left(\bigcup \mathscr{G}_{i-1}\right)$. At least one set in $\left\{I_{1}, \ldots, I_{k}\right\}$ is infinite. Indeed, if every $I_{j}$ were finite, then there would exist an integer $i$ such that $i \notin I_{j}$ for each $j \in\{1, \ldots, k\}$. Hence, 
there would exists a group $\mathscr{G}_{i-1}$ such that $\mathscr{D}_{1}\left(\bigcup \mathscr{G}_{i-1}\right) \neq \Delta_{2}^{j}\left(\bigcup \mathscr{G}_{i-1}\right)$ for each $j \in\{1, \ldots, k\}$. However, this implies $s_{1} \chi_{\mathrm{PB}, \mathrm{gbg},=}^{i} s_{2}$, which whould contradict the assumption $s_{1} \sim^{\prime} s_{2}$. Let $j^{\prime}$ be such that $I_{j^{\prime}}$ is infinite. We have that:

$$
\begin{aligned}
\mathscr{D}_{1}(\bigcup \mathscr{G}) & =\inf _{i \in \mathbb{N}} \mathscr{D}_{1}\left(\bigcup \mathscr{G}_{i}\right)=\inf _{i \in I_{j^{\prime}}} \mathscr{D}_{1}\left(\bigcup \mathscr{G}_{i}\right)= \\
& =\inf _{i \in I_{j^{\prime}}} \Delta_{2}^{j^{\prime}}\left(\bigcup \mathscr{G}_{i}\right)=\inf _{i \in \mathbb{N}} \Delta_{2}^{j^{\prime}}\left(\bigcup \mathscr{G}_{i}\right)=\Delta_{2}^{j^{\prime}}(\bigcup \mathscr{G})
\end{aligned}
$$

where the second and the fourth equalities derive from the fact that $\left(\mathscr{D}_{1}\left(\bigcup \mathscr{G}_{i}\right)\right)_{i \in I_{j^{\prime}}}$ and $\left(\Delta_{2}^{j^{\prime}}\left(\bigcup \mathscr{G}_{i}\right)\right)_{i \in I_{j^{\prime}}}$ are infinite subsequences of the non-increasing sequences $\left(\mathscr{D}_{1}\left(\bigcup \mathscr{G}_{i}\right)\right)_{i \in \mathbb{N}}$ and $\left(\Delta_{2}^{j^{\prime}}\left(\bigcup \mathscr{G}_{i}\right)\right)_{i \in \mathbb{N}}$, respectively, and therefore they have pairwise the same infimum. In conclusion, transition $s_{1} \stackrel{a}{\longrightarrow} \mathscr{D}_{1}$ is matched by transition $s_{2} \stackrel{a}{\longrightarrow} \Delta_{2}^{j^{\prime}}$ with respect to $\mathscr{G}$.

The second step consists of showing that two states are equated by $\sim_{\mathrm{PB}, \mathrm{gbg},=}^{i}$ iff they satisfy the same formulae in $\mathbb{F}_{\mathrm{PML}}^{i}{ }_{\exists, \mathrm{I}}$, which is the set of formulae in $\mathbb{F}_{\mathrm{PML}_{\exists, \mathrm{I}}}$ whose maximum number of nested diamond operators is at most $i$. We denote by $\mathscr{F}_{\mathrm{PML}}^{i}{ }_{\exists, \mathrm{I}}(s)$ the set of formulae in $\mathbb{F}_{\mathrm{PML}}^{i}$ s.I satisfied by state $s$.

Lemma 2 Let $(S, A, \longrightarrow)$ be an image-finite NPLTS satisfying the minimal probability assumption. Let $s_{1}, s_{2} \in S$. Then for all $i \in \mathbb{N}$ :

$$
s_{1} \sim_{\mathrm{PB}, \mathrm{gbg},=}^{i} s_{2} \Longleftrightarrow \mathscr{F}_{\mathrm{PML}_{\exists, \mathrm{I}}}^{i}\left(s_{1}\right)=\mathscr{F}_{\mathrm{PML}_{\exists, \mathrm{I}}}^{i}\left(s_{2}\right)
$$

Proof Given an image-finite NPLTS $(S, A, \longrightarrow)$ satisfying the minimal probability assumption, and given $s_{1}, s_{2} \in S$, we proceed by induction on $i \in \mathbb{N}$.

Let $i=0$. Since $\sim_{\mathrm{PB}, \mathrm{gbg},=}^{0}=S \times S$ and $\mathscr{F}_{\mathrm{PML}_{\exists, \mathrm{I}}}^{0}(s)=\left\{\phi \in \mathbb{F}_{\mathrm{PML}}^{0} \mid \phi \equiv\right.$ true $\}$ for all $s \in S$, it trivially holds that:

$$
s_{1} \sim_{\mathrm{PB}, \mathrm{gbg},=}^{0} s_{2} \Longleftrightarrow \mathscr{F}_{\mathrm{PML}_{\exists, \mathrm{I}}}^{0}\left(s_{1}\right)=\mathscr{F}_{\mathrm{PML}_{\exists, \mathrm{I}}}^{0}\left(s_{2}\right)
$$

Let $i \in \mathbb{N}$ and suppose that for all $j=0, \ldots, i$ :

$$
s_{1} \sim_{\mathrm{PB}, \mathrm{gbg},=}^{j} s_{2} \Longleftrightarrow \mathscr{F}_{\mathrm{PML}_{\exists, \mathrm{I}}}^{j}\left(s_{1}\right)=\mathscr{F}_{\mathrm{PML}_{\exists, \mathrm{I}}}^{j}\left(s_{2}\right)
$$

We prove both implications for $i+1$ by reasoning on their corresponding contrapositive statements, i.e., we prove that:

$$
\mathscr{F}_{\mathrm{PML}_{\exists, \mathrm{I}}}^{i+1}\left(s_{1}\right) \neq \mathscr{F}_{\mathrm{PML}}^{i+1}\left(s_{2}\right) \Longleftrightarrow s_{1} \chi_{\mathrm{PB}, \mathrm{gbg},=}^{i+1} s_{2}
$$

$(\Longrightarrow)$ If $\mathscr{F}_{\mathrm{PML}_{\exists, \mathrm{I}}}^{i+1}\left(s_{1}\right) \neq \mathscr{F}_{\mathrm{PML}_{\exists, \mathrm{I}}^{i+1}}^{i+1}\left(s_{2}\right)$, then there are two cases:

- If $\mathscr{F}_{\mathrm{PML}}^{i}\left(s_{1}\right) \neq \mathscr{F}_{\mathrm{PML}}^{i}{ }_{\exists, \mathrm{I}}\left(s_{2}\right)$, then by the induction hypothesis it holds that $s_{1} \chi_{\mathrm{PB}, \mathrm{gbg},=}^{i} s_{2}$ and hence $s_{1} \chi_{\mathrm{PB}, \mathrm{gbg},=}^{i+1} s_{2}$.

- If $\mathscr{F}_{\mathrm{PML}}^{i}\left(s_{\exists, \mathrm{I}}\right)=\mathscr{F}_{\mathrm{PML}_{\exists, \mathrm{I}}}^{i}\left(s_{2}\right)$, then from $\mathscr{F}_{\mathrm{PML}_{\exists, \mathrm{I}}}^{i+1}\left(s_{1}\right) \neq \mathscr{F}_{\mathrm{PML}_{\exists, \mathrm{I}}}^{i+1}\left(s_{2}\right)$ it follows that there exists $\phi \in \mathbb{F}_{\mathrm{PML}}^{i+1}$ such that $s_{1} \in \mathscr{M}_{\mathrm{PML}_{\exists, \mathrm{I}}}[[\phi]]$ and $s_{2} \notin \mathscr{M}_{\mathrm{PML}_{\exists, \mathrm{I}}}[[\phi]]$. We now proceed by induction on the syntactical structure of $\phi$. Here we only consider the case $\phi=$ $\langle a\rangle_{\left[p_{1}, p_{2}\right]} \phi^{\prime}$ because the other cases are routine.

From $s_{1} \in \mathscr{M}_{\mathrm{PML}_{\exists, \mathrm{I}}}\left[\left\langle\langle a\rangle_{\left[p_{1}, p_{2}\right]} \phi^{\prime}\right]\right]$ and $\left.s_{2} \notin \mathscr{M}_{\mathrm{PML}_{\exists, \mathrm{I}}}\left[\langle a\rangle_{\left[p_{1}, p_{2}\right]} \phi^{\prime}\right]\right]$, it follows that:

- $p_{1} \leq \mathscr{D}_{1}\left(\mathscr{M}_{\mathrm{PML}_{\exists, \mathrm{I}}}\left[\left[\phi^{\prime}\right]\right]\right) \leq p_{2}$ for some $\mathscr{D}_{1}$ such that $s_{1} \stackrel{a}{\longrightarrow} \mathscr{D}_{1}$.

- $\mathscr{D}_{2}\left(\mathscr{M}_{\mathrm{PML}_{\exists, \mathrm{I}}}\left[\left[\phi^{\prime}\right]\right]\right)<p_{1}$ or $\mathscr{D}_{2}\left(\mathscr{M}_{\mathrm{PML}_{\exists, \mathrm{I}}}\left[\left[\phi^{\prime}\right]\right]\right)>p_{2}$ for all $\mathscr{D}_{2}$ such that $s_{2} \stackrel{a}{\longrightarrow} \mathscr{D}_{2}$.

Since $\phi^{\prime} \in \mathbb{F}_{\mathrm{PML}}^{i}$, by the induction hypothesis there exists $\mathscr{G} \in 2^{S / \sim_{\mathrm{PB}, \mathrm{gbg},=}^{i}}$ such that $\bigcup_{C \in \mathscr{G}} C=\mathscr{M}_{\mathrm{PML}_{\exists, \mathrm{I}}}\left[\left[\phi^{\prime}\right]\right]$. Then:

- $\mathscr{D}_{1}(\bigcup \mathscr{G})=q \in \mathbb{R}_{\left[p_{1}, p_{2}\right]}$.

- $\mathscr{D}_{2}(\bigcup \mathscr{G}) \neq q$ for all $\mathscr{D}_{2}$ such that $s_{2} \stackrel{a}{\longrightarrow} \mathscr{D}_{2}$. 
Therefore $s_{1} \chi_{\mathrm{PB}, \mathrm{gbg},=}^{i+1} s_{2}$.

$(\Longleftarrow)$ If $s_{1} \mathcal{\chi}_{\mathrm{PB}, \mathrm{gbg},=}^{i+1} s_{2}$, then there are two cases:

- If $s_{1} \chi_{\mathrm{PB}, \mathrm{gbg},=}^{i} s_{2}$, then by the induction hypothesis it holds that $\mathscr{F}_{\mathrm{PML}}^{i}\left(s_{1, \mathrm{I}}\right) \neq \mathscr{F}_{\mathrm{PML}}^{i}\left(s_{2}\right)$ and hence $\mathscr{F}_{\mathrm{PML}}^{i+1}\left(s_{1}\right) \neq \mathscr{F}_{\mathrm{PML}_{\exists, \mathrm{I}}}^{i+1}\left(s_{2}\right)$.

- If $s_{1} \sim_{\mathrm{PB}, \mathrm{gbg},=}^{i} s_{2}$, then from $s_{1} \chi_{\mathrm{PB}, \mathrm{gbg},=}^{i+1} s_{2}$ it follows that there exist $p \in \mathbb{R}_{[0,1]}$ and $\mathscr{G} \in 2^{S / \sim_{\mathrm{PB}, \mathrm{gbg},=}^{i}}$ such that:

- $\mathscr{D}_{1}(\bigcup \mathscr{G})=$ for some $\mathscr{D}_{1}$ such that $s_{1} \stackrel{a}{\longrightarrow} \mathscr{D}_{1}$.

- $\mathscr{D}_{2}(\bigcup \mathscr{G}) \neq$ p for all $\mathscr{D}_{2}$ such that $s_{2} \stackrel{a}{\longrightarrow} \mathscr{D}_{2}$.

Let $\mathscr{G}_{1}=\left\{C \in S / \sim_{\mathrm{PB}, \mathrm{gbg},=}^{i} \mid \mathscr{D}_{1}(C)>0\right\}$ and $\mathscr{G}_{2}=\left\{C \in S / \sim_{\mathrm{PB}, \mathrm{gbg},=}^{i} \mid \exists \mathscr{D}_{2} . s_{2} \stackrel{a}{\longrightarrow} \mathscr{D}_{2} \wedge\right.$ $\left.\mathscr{D}_{2}(C)>0\right\}$. Thanks to the assumptions of image finiteness and minimal probability, both $\mathscr{G}_{1}$ and $\mathscr{G}_{2}$ are finite.

By the induction hypothesis, there exists a distinguishing formula $\phi_{<C_{1}, C_{2}>} \in \mathbb{F}_{\mathrm{PML}_{\exists, \mathrm{I}}}^{i}$ for all $C_{1}$ and $C_{2}$ in $S / \sim_{\mathrm{PB}, \mathrm{gbg},=}^{i}$ such that $C_{1} \neq C_{2}$, i.e.:

Then:

$$
\begin{aligned}
& C_{1} \subseteq \mathscr{M}_{\mathrm{PML}_{\exists, \mathrm{I}}}\left[\left[\phi_{<C_{1}, C_{2}>}\right]\right] \\
& \left.C_{2} \subseteq S \backslash \mathscr{M}_{\mathrm{PML}_{\exists, \mathrm{I}}}\left[\phi_{<C_{1}, C_{2}>}\right]\right]
\end{aligned}
$$

$$
\phi_{\mathscr{G}}=\bigvee_{C \in \mathscr{G}}\left(\bigwedge_{C_{1} \in \mathscr{G}_{1} \backslash\{C\}} \phi_{<C, C_{1}>} \wedge \bigwedge_{C_{2} \in \mathscr{G}_{2} \backslash\{C\}} \phi_{<C, C_{2}>}\right)
$$

where $\bigvee_{i \in I} \phi_{i}=\neg \bigwedge_{i \in I} \neg \phi_{i}$ for I finite and $\bigwedge_{i \in I} \phi_{i}=$ true for $I=\emptyset$, yields a distinguishing formula for $s_{1}$ and $s_{2}$ because:

- $s_{1} \in \mathscr{M}_{\mathrm{PML}_{\exists, \mathrm{I}}}\left[\left\langle\langle a\rangle_{[p, p]} \phi_{\mathscr{G}}\right]\right]$

- $\left.s_{2} \notin \mathscr{M}_{\mathrm{PML}_{\exists, \mathrm{I}}}\left[\langle a\rangle_{[p, p]} \phi \mathscr{G}\right]\right]$.

Since $\langle a\rangle_{[p, p]} \phi_{\mathscr{G}} \in \mathbb{F}_{\mathrm{PML}_{\exists, \mathrm{I}}}^{i+1}$, we derive that $\mathscr{F}_{\mathrm{PML}_{\exists, \mathrm{I}}}^{i+1}\left(s_{1}\right) \neq \mathscr{F}_{\mathrm{PML}_{\exists, \mathrm{I}}}^{i+1}\left(s_{2}\right)$.

Theorem 1 Let $(S, A, \longrightarrow)$ be an image-finite NPLTS satisfying the minimal probability assumption. Let $s_{1}, s_{2} \in S$. Then:

$$
s_{1} \sim \mathrm{PB}, \mathrm{gbg},=s_{2} \Longleftrightarrow s_{1} \sim \sim_{\mathrm{PML}_{\exists, \mathrm{I}}} s_{2}
$$

The result established by Thm. 1 would not hold if $\mathrm{PML}_{\exists, \geq}$ or $\mathrm{PML}_{\exists, \leq}$ were used instead of PML $\mathrm{PMII}_{\exists}$. For instance, the two states $s_{1}$ and $s_{2}$ in Fig. 2 are not related by $\mathrm{PML}_{\exists, \mathrm{I}}$ as only $s_{1}$ satisfies the $\mathrm{PML}_{\exists, \mathrm{I}}$ formula $\langle\text { offer }\rangle_{[0.5,0.5]}\langle\text { head }\rangle_{[1,1]}$ true. However, those two states cannot be distinguished by any $\mathrm{PML}_{\exists, \geq}$ or $\mathrm{PML}_{\exists, \leq}$ formula, because the states enabling head (resp. tail) have the same extremal probabilities 0.7 and 0.3 of being reached in both systems. Indeed, as can be noted 0.5 , the only nonzero value occurring in the target distribution of the central transition of $s_{1}$, belongs to the interval $[0.3,0.7]$ determined by the target distribution of each of the other transitions.

Following the same proof pattern, we can show that the relations $\sim_{\mathrm{PB}, \mathrm{gbg}, \leq}$ and $\sim_{\mathrm{PB}, \mathrm{gbg}, \geq}$ are respectively characterized by $\mathrm{PML}_{\exists, \geq}$ and $\mathrm{PML}_{\exists, \leq}$.

Theorem 2 Let $(S, A, \longrightarrow)$ be an image-finite NPLTS satisfying the minimal probability assumption. Let $s_{1}, s_{2} \in S$. Then:

$$
\begin{aligned}
& s_{1} \sim_{\mathrm{PB}, \mathrm{gbg}, \leq s_{2}} \Longleftrightarrow s_{1} \sim_{\mathrm{PML}_{\exists, \geq}} s_{2} \\
& s_{1} \sim_{\mathrm{PB}, \mathrm{gbg}, \geq} s_{2} \Longleftrightarrow s_{1} \sim_{\mathrm{PML}_{\exists, \leq}} s_{2}
\end{aligned}
$$


Proof The proof of the first result is similar to the proof of Thm. 1 - based on Lemmata 1 and $2-u p$ to the use of $\leq$ in place of $=$ when comparing the probabilities of reaching a group of equivalence classes and the use of $\sim_{\mathrm{PB}, \mathrm{gbg}, \leq \leq}^{i}, \mathscr{F}_{\mathrm{PML}_{\exists, \geq}}^{i}, \mathscr{M}_{\mathrm{PML}_{\exists, \geq}}$, and $\langle\text { a }\rangle_{p}$ in place of $\sim_{\mathrm{PB}, \mathrm{gbg},=}^{i}, \mathscr{F}_{\mathrm{PML}}^{i}, \mathscr{M}_{\mathrm{PML}_{\exists, \mathrm{I}}}$, and $\langle a\rangle_{\left[p_{1}, p_{2}\right]}$ wherever necessary.

In particular, for the induction step of Lemma 2 we point out that:

- In the $(\Longrightarrow)$ part, from $s_{1} \in \mathscr{M}_{\mathrm{PML}_{\exists, \geq}}\left[\left[\langle a\rangle_{p} \phi^{\prime}\right]\right]$ and $s_{2} \notin \mathscr{M}_{\mathrm{PML}_{\exists, \geq}}\left[\left[\langle a\rangle_{p} \phi^{\prime}\right]\right]$, it follows that:

- $\left.\mathscr{D}_{1}\left(\mathscr{M}_{\mathrm{PML}_{\exists,>}}\left[\phi^{\prime}\right]\right]\right) \geq$ p for some $\mathscr{D}_{1}$ such that $s_{1} \stackrel{a}{\longrightarrow} \mathscr{D}_{1}$.

- $\mathscr{D}_{2}\left(\mathscr{M}_{\mathrm{PML}_{\exists, \geq}}\left[\phi^{\prime}\right]\right)<$ p for all $\mathscr{D}_{2}$ such that $s_{2} \stackrel{a}{\longrightarrow} \mathscr{D}_{2}$.

Since $\phi^{\prime} \in \mathbb{F}_{\mathrm{PML}}^{i}$, by the induction hypothesis there exists $\mathscr{G} \in 2^{S / \sim_{\mathrm{PB}, \mathrm{gbg}, \leq}^{i}}$ such that $\left.\cup_{C \in \mathscr{G}} C=\mathscr{M}_{\mathrm{PML}_{\exists, \geq}}\left[\phi^{\prime}\right]\right]$. Then:

- $\mathscr{D}_{1}(\bigcup \mathscr{G}) \geq p$.

- $\mathscr{D}_{2}(\bigcup \mathscr{G})<$ p for all $\mathscr{D}_{2}$ such that $s_{2} \stackrel{a}{\longrightarrow} \mathscr{D}_{2}$.

- In the $(\Longleftarrow)$ part, if $s_{1} \sim_{\mathrm{PB}, \mathrm{gbg}, \leq}^{i} s_{2}$, then there exist $p \in \mathbb{R}_{[0,1]}$ and $\mathscr{G} \in 2^{S / \sim_{\mathrm{PB}, \mathrm{gbg}, \leq}^{i}}$ such that:

- $\mathscr{D}_{1}(\bigcup \mathscr{G})=$ p for some $\mathscr{D}_{1}$ such that $s_{1} \stackrel{a}{\longrightarrow} \mathscr{D}_{1}$.

- $\mathscr{D}_{2}(\bigcup \mathscr{G})<$ p for all $\mathscr{D}_{2}$ such that $s_{2} \stackrel{a}{\longrightarrow} \mathscr{D}_{2}$.

The distinguishing formula in $\mathbb{F}_{\mathrm{PML}_{\exists, \geq}^{i+1}}$ for $s_{1}$ and $s_{2}$ is then $\langle a\rangle_{p} \phi_{\mathscr{G}}$.

The proof of the second result is similar to the proof of the first one up to the use of $\geq$ in place of $\leq$ and $>$ in place of $<$ wherever necessary.

It is easy to see that $\sim_{\mathrm{PB}, \mathrm{gbg},=}^{\mathrm{ct}}, \sim_{\mathrm{PB}, \mathrm{gbg}, \leq}^{\mathrm{ct}}$, and $\sim_{\mathrm{PB}, \mathrm{gbg}, \geq}^{\mathrm{ct}}$ are respectively characterized by the logics $\mathrm{PML}_{\exists, \mathrm{I}}^{\mathrm{ct}}, \mathrm{PML}_{\exists, \geq}^{\mathrm{ct}}$, and $\mathrm{PML}_{\exists, \leq}^{\mathrm{ct}}$ in which the interpretation of the diamond operator relies on combined transitions instead of ordinary ones.

\section{Variants and Comparisons for Group-by-Group Probabilistic Bisimilarities}

For the three group-by-group probabilistic bisimilarities, we have shown characterizations based on the three existentially interpreted variants of PML. In this section, we present further motivations behind their definition (Sect. 5.1), three variants relying on extremal probabilities that are characterized by the three universally interpreted variants of PML (Sect. 5.2), and a comparison with the probabilistic bisimilarities of [42] that are characterized by PCTL/PCTL* (Sect. 5.3).

\subsection{Class-by-Class Probabilistic Bisimilarities}

Instead of using groups of equivalence classes as in Def. 6, the most natural way of presenting our approach would be to introduce class-by-class variants of $\sim_{\mathrm{PB} \text {,dis }}$. These are obtained by simply anticipating the quantification over equivalence classes of target states in Def. 4. In the definition below, as usual we let $\bowtie \in\{=, \leq, \geq\}$.

Definition 7 Let $(S, A, \longrightarrow)$ be an NPLTS. An equivalence relation $\mathscr{B}$ over $S$ is a $\bowtie$-classby-class probabilistic bisimulation iff, whenever $\left(s_{1}, s_{2}\right) \in \mathscr{B}$, then for all actions $a \in A$ and 


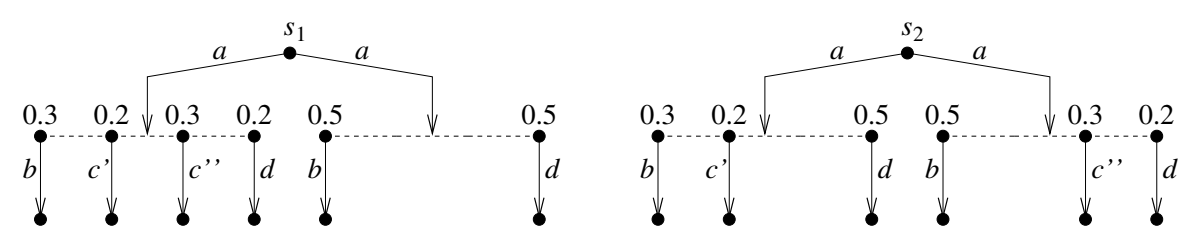

Fig. 3 Models related by $\sim_{\mathrm{PB}, \mathrm{cbc},=}$ and distinguished by all PML variants

for all equivalence classes $C \in S / \mathscr{B}$ it holds that for each $s_{1} \stackrel{a}{\longrightarrow} \mathscr{D}_{1}$ there exists $s_{2} \stackrel{a}{\longrightarrow} \mathscr{D}_{2}$ such that $\mathscr{D}_{1}(C) \bowtie \mathscr{D}_{2}(C)$. We denote by $\sim_{\mathrm{PB}, \text { cbc }, \bowtie}$ the largest $\bowtie$-class-by-class probabilistic bisimulation.

The problem with Def. 7 is that the resulting relations $\sim_{\mathrm{PB}, \mathrm{cbc}, \bowtie}$ are too coarse. For example, in Fig. 3 it holds that $s_{1} \sim_{\mathrm{PB}, \mathrm{cbc},=} s_{2}$, as witnessed by the $=$-class-by-class probabilistic bisimulation that pairs states with identically labeled transitions. However, after performing $a$, from $s_{2}$ it is always possible to reach a state in which $c^{\prime}$ or $c^{\prime \prime}$ is enabled, whereas this is not the case from $s_{1}$. Indeed, the relation $\sim_{\mathrm{PB}, \mathrm{gbg},=}=$ would tell apart $s_{1}$ and $s_{2}$, because the rightmost (resp. leftmost) transition of $s_{1}$ reaches the group of states enabling $c^{\prime}$ or $c^{\prime \prime}$ with probability 0 (resp. 1), while the two transitions of $s_{2}$ reach the same group with probabilities 0.2 and 0.3 , respectively.

From a modal logic perspective, none of the relations $\sim_{\mathrm{PB}, \mathrm{cbc}, \bowtie}$ is characterized by the PML variants of Sect. 3. For instance, in Fig. 3 it holds that only $s_{1}$ satisfies the following existentially interpreted formulae:

$$
\begin{aligned}
& \mathrm{PML}_{\exists, \geq}:\langle a\rangle_{0.5}\left(\left\langle c^{\prime}\right\rangle_{1} \text { true } \vee\left\langle c^{\prime \prime}\right\rangle_{1} \text { true }\right) \\
& \mathrm{PML}_{\exists, \leq}:\langle a\rangle_{0}\left(\left\langle c^{\prime}\right\rangle_{1} \text { true } \vee\left\langle c^{\prime \prime}\right\rangle_{1} \text { true }\right) \\
& \mathrm{PML}_{\exists, \mathrm{I}}: \quad \neg\langle a\rangle_{[0.2,0.3]}\left(\left\langle c^{\prime}\right\rangle_{[1,1]} \text { true } \vee\left\langle c^{\prime \prime}\right\rangle_{[1,1]} \text { true }\right)
\end{aligned}
$$

while only $s_{2}$ satisfies the following universally interpreted formulae:

$$
\begin{aligned}
& \operatorname{PML}_{\forall, \geq}:\langle a\rangle_{0.7}\left(\langle b\rangle_{1} \text { true } \vee\langle d\rangle_{1} \text { true }\right) \\
& \operatorname{PML}_{\forall, \leq}:\langle a\rangle_{0.8}\left(\langle b\rangle_{1} \text { true } \vee\langle d\rangle_{1} \text { true }\right) \\
& \operatorname{PML}_{\forall, \mathrm{I}}: \quad\langle a\rangle_{[0.7,0.8]}\left(\langle b\rangle_{[1,1]} \text { true } \vee\langle d\rangle_{[1,1]} \text { true }\right)
\end{aligned}
$$

where $\phi_{1} \vee \phi_{2}$ stands for $\neg\left(\neg \phi_{1} \wedge \neg \phi_{2}\right)$. The presence of the logical disjunction in the distinguishing formulae above clearly indicates that - having anticipated the quantification over the target states - it is necessary to group equivalence classes together if one wants to obtain the same identifications as the equivalences induced by the variants of PML.

\subsection{Extremal Probabilities and Universal Interpretations of PML}

The three group-by-group probabilistic bisimilarities of Def. 6 are respectively characterized by the three existentially interpreted variants of PML. We consider below three variants of the group-by-group approach in which only the supremum $(\sqcup)$ and/or the infimum $(\sqcap)$ of the probabilities of reaching a certain group after the execution of a certain action are considered. It turns out that the resulting relations are respectively characterized by the three universally interpreted variants of PML.

Definition 8 Let $(S, A, \longrightarrow)$ be an NPLTS. An equivalence relation $\mathscr{B}$ over $S$ is a $\sqcup \sqcap$-groupby-group probabilistic bisimulation iff, whenever $\left(s_{1}, s_{2}\right) \in \mathscr{B}$, then for all actions $a \in A$ and groups of equivalence classes $\mathscr{G} \in 2^{S / \mathscr{B}}$ it holds that $s_{1} \stackrel{a}{\longrightarrow}$ iff $s_{2} \stackrel{a}{\longrightarrow}$ and: 


$$
\begin{aligned}
& \bigsqcup_{s_{1} \stackrel{a}{\longrightarrow} \mathscr{D}_{1}} \mathscr{D}_{1}(\bigcup \mathscr{G})=\bigsqcup_{s_{2} \stackrel{a}{\longrightarrow} \mathscr{D}_{2}} \mathscr{D}_{2}(\bigcup \mathscr{G}) \\
& \prod \quad \mathscr{D}_{1}(\bigcup \mathscr{G})=\prod \quad \mathscr{D}_{2}(\bigcup \mathscr{G}) \\
& s_{1} \stackrel{a}{\longrightarrow} \mathscr{D}_{1} \quad s_{2} \stackrel{a}{\longrightarrow} \mathscr{D}_{2}
\end{aligned}
$$

We denote by $\sim_{\mathrm{PB}, \text { gbg }, \sqcup \sqcap}$ the largest $\sqcup \sqcap$-group-by-group probabilistic bisimulation.

Theorem 3 Let $(S, A, \longrightarrow)$ be an image-finite NPLTS satisfying the minimal probability assumption. Let $s_{1}, s_{2} \in S$. Then:

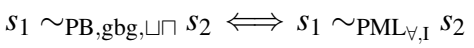

Proof Similar to the proof of Thm. 1 - based on Lemmata 1 and 2 - up to the use of $\bigsqcup$ and $\Pi$ in place of individual values when comparing the probabilities of reaching a group of equivalence classes and the use of $\sim_{\mathrm{PB}, \mathrm{gbg}, \sqcup \sqcap}^{i}, \mathscr{F}_{\mathrm{PML}}^{i}$, and $\mathscr{M}_{\mathrm{PML}_{\forall, \mathrm{I}}}$ in place of $\sim_{\mathrm{PB}, \mathrm{gbg},=}^{i}$, $\mathscr{F}_{\mathrm{PML}}^{i}{ }_{\exists, \mathrm{I}}$, and $\mathscr{M}_{\mathrm{PML}_{\exists, \mathrm{I}}}$ wherever necessary.

In particular, for the induction step of Lemma 2 we point out that:

- In the $(\Longrightarrow)$ part, from $s_{1} \in \mathscr{M}_{\mathrm{PML}_{\forall, \mathrm{I}}}\left[\left\langle\langle a\rangle_{\left[p_{1}, p_{2}\right]} \phi^{\prime}\right]\right]$ and $s_{2} \notin \mathscr{M}_{\mathrm{PML}_{\forall, \mathrm{I}}}\left[\left[\langle a\rangle_{\left[p_{1}, p_{2}\right]} \phi^{\prime}\right]\right]$, it follows that:

- $s_{1} \stackrel{a}{\longrightarrow}$ and $p_{1} \leq \mathscr{D}_{1}\left(\mathscr{M}_{\mathrm{PML}_{\forall, \mathrm{I}}}\left[\left[\phi^{\prime}\right]\right]\right) \leq p_{2}$ for all $\mathscr{D}_{1}$ such that $s_{1} \stackrel{a}{\longrightarrow} \mathscr{D}_{1}$.

- $s_{2} \stackrel{a}{\rightarrow}$ or $\mathscr{D}_{2}\left(\mathscr{M}_{\mathrm{PML}_{\forall, \mathrm{I}}}\left[\phi^{\prime}\right]\right)<p_{1}$ or $\mathscr{D}_{2}\left(\mathscr{M}_{\mathrm{PML}_{\forall, \mathrm{I}}}\left[\left[\phi^{\prime}\right]\right]\right)>p_{2}$ for some $\mathscr{D}_{2}$ such that $s_{2} \stackrel{a}{\longrightarrow} \mathscr{D}_{2}$.

Since $\phi^{\prime} \in \mathbb{F}_{\mathrm{PML}}^{i}$, by the induction hypothesis there exists $\mathscr{G} \in 2^{S / \sim_{\mathrm{PB}, \mathrm{ggg}, \sqcup \sqcap}^{i}}$ such that $\cup_{C \in \mathscr{G}} C=\mathscr{M}_{\mathrm{PML}_{\forall, \mathrm{I}}}\left[\left[\phi^{\prime}\right]\right]$. Then:

- $s_{1} \stackrel{a}{\longrightarrow}$ and $\underset{s_{1} \stackrel{a}{\longrightarrow} \mathscr{D}_{1}}{\bigsqcup} \mathscr{D}_{1}(\bigcup \mathscr{G})=q^{\prime \prime}, \prod_{s_{1} \stackrel{a}{\longrightarrow} \mathscr{D}_{1}} \mathscr{D}_{1}(\bigcup \mathscr{G})=q^{\prime}$ with $q^{\prime}, q^{\prime \prime} \in \mathbb{R}_{\left[p_{1}, p_{2}\right]}$ such that $q^{\prime} \leq q^{\prime \prime}$.

- $s_{2} \stackrel{a}{\rightarrow}$ or $\underset{s_{2} \stackrel{a}{\longrightarrow} \mathscr{D}_{2}}{\bigsqcup} \mathscr{D}_{2}(\bigcup \mathscr{G})>q^{\prime \prime}$ or $\prod_{s_{2} \stackrel{a}{\longrightarrow} \mathscr{D}_{2}} \mathscr{D}_{2}(\bigcup \mathscr{G})<q^{\prime}$.

- In the $(\Longleftarrow)$ part, if $s_{1} \sim_{\mathrm{PB}, \mathrm{gbg}, \sqcup \sqcap}^{i} s_{2}$, then there exist $p_{1}^{\prime}, p_{1}^{\prime \prime}, p_{2}^{\prime}, p_{2}^{\prime \prime} \in \mathbb{R}_{[0,1]}$ - with $p_{1}^{\prime} \leq p_{1}^{\prime \prime}$ and $p_{2}^{\prime} \leq p_{2}^{\prime \prime}-$ and $\mathscr{G} \in 2^{S / \sim_{\mathrm{PB}, \mathrm{gbg}, \sqcup \sqcap}^{i}}$ such that:

- $s_{1} \stackrel{a}{\longrightarrow}$ and $\underset{s_{1} \stackrel{a}{\longrightarrow} \mathscr{D}_{1}}{\bigsqcup} \mathscr{D}_{1}(\bigcup \mathscr{G})=p_{1}^{\prime \prime}, \quad \prod_{s_{1} \stackrel{a}{\longrightarrow} \mathscr{D}_{1}} \mathscr{D}_{1}(\bigcup \mathscr{G})=p_{1}^{\prime}$.

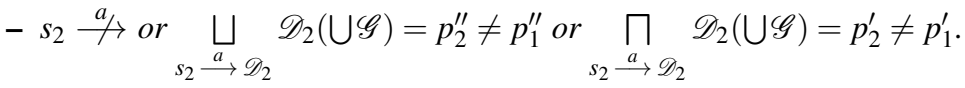

Let $\mathscr{G}_{1}=\left\{C \in S / \sim_{\mathrm{PB}, \mathrm{gbg}, \sqcup \sqcap}^{i} \mid \exists \mathscr{D}_{1} . s_{1} \stackrel{a}{\longrightarrow} \mathscr{D}_{1} \wedge \mathscr{D}_{1}(C)>0\right\}$ and $\mathscr{G}_{2}=\left\{C \in S / \sim_{\mathrm{PB}, \mathrm{gbg}, \sqcup \sqcap}^{i}\right.$ $\left.\mid \exists \mathscr{D}_{2} \cdot s_{2} \stackrel{a}{\longrightarrow} \mathscr{D}_{2} \wedge \mathscr{D}_{2}(C)>0\right\}$. The distinguishing formula in $\mathbb{F}_{\mathrm{PML}_{\forall, \mathrm{I}}^{i+1}}^{i+}$ for $s_{1}$ and $s_{2}$ is then:

- $\langle a\rangle_{\left[p_{1}^{\prime}, p_{1}^{\prime \prime}\right]} \phi_{G}$ if $s_{2} \stackrel{a}{\rightarrow}$ or it is not the case that $p_{1}^{\prime} \leq p_{2}^{\prime}$ and $p_{2}^{\prime \prime} \leq p_{1}^{\prime \prime}$.

- $\langle a\rangle_{\left[p_{2}^{\prime}, p_{2}^{\prime \prime}\right]} \phi_{G}$ if $s_{2} \stackrel{a}{\longrightarrow}$ and it is the case that $p_{1}^{\prime} \leq p_{2}^{\prime}$ and $p_{2}^{\prime \prime} \leq p_{1}^{\prime \prime}$.

Definition 9 Let $(S, A, \longrightarrow)$ be an NPLTS and \# $\in\{\sqcup, \sqcap\}$. An equivalence relation $\mathscr{B}$ over $S$ is a \#-group-by-group probabilistic bisimulation iff, whenever $\left(s_{1}, s_{2}\right) \in \mathscr{B}$, then for all actions $a \in A$ and groups of equivalence classes $\mathscr{G} \in 2^{S / \mathscr{B}}$ it holds that $s_{1} \stackrel{a}{\longrightarrow}$ iff $s_{2} \stackrel{a}{\longrightarrow}$ and:

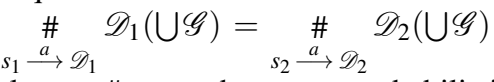

We denote by $\sim_{\mathrm{PB}, \text { gbg,\# }}$ the largest \#-group-by-group probabilistic bisimulation. 
Theorem 4 Let $(S, A, \longrightarrow)$ be an image-finite NPLTS satisfying the minimal probability assumption. Let $s_{1}, s_{2} \in S$. Then:

$$
\begin{aligned}
& s_{1} \sim_{\mathrm{PB}, \mathrm{gbg}, \sqcup s_{2}} \Longleftrightarrow s_{1} \sim \sim_{\mathrm{PML}}, \leq s_{2} \\
& s_{1} \sim \mathrm{PB}, \mathrm{gbg}, \sqcap s_{2} \Longleftrightarrow s_{1} \sim \sim_{\mathrm{PML}}, \geq
\end{aligned}
$$

Proof The proof of the first result is similar to the proof of Thm. 1 - based on Lemmata 1 and 2 - up to the use of $\bigsqcup$ in place of individual values when comparing the probabilities of reaching a group of equivalence classes and the use of $\sim_{\mathrm{PB}, \mathrm{gbg}, \sqcup}^{i}, \mathscr{F}_{\mathrm{PML}_{\forall, \leq}^{i}}$, and $\mathscr{M}_{\mathrm{PML}_{\forall, \leq}}$ in place of $\sim_{\mathrm{PB}, \mathrm{gbg},=}^{i}, \mathscr{F}_{\mathrm{PML}}^{i}$, and $\mathscr{M}_{\mathrm{PML}}{ }_{\exists, \mathrm{I}}$ wherever necessary.

In particular, for the induction step of Lemma 2 we point out that:

- In the $(\Longrightarrow)$ part, from $s_{1} \in \mathscr{M}_{\mathrm{PML}_{\forall, \leq}}\left[\left[\langle a\rangle_{p} \phi^{\prime}\right]\right]$ and $s_{2} \notin \mathscr{M}_{\mathrm{PML}_{\forall, \leq}}\left[\left[\langle a\rangle_{p} \phi^{\prime}\right]\right]$, it follows that:

- $s_{1} \stackrel{a}{\longrightarrow}$ and $\mathscr{D}_{1}\left(\mathscr{M}_{\mathrm{PML}_{\forall}, \leq}\left[\left[\phi^{\prime}\right]\right]\right) \leq$ p for all $\mathscr{D}_{1}$ such that $s_{1} \stackrel{a}{\longrightarrow} \mathscr{D}_{1}$.

- $s_{2} \stackrel{a}{\rightarrow}$ or $\mathscr{D}_{2}\left(\mathscr{M}_{\mathrm{PML}_{\forall, \leq}}\left[\left[\phi^{\prime}\right]\right]\right)>$ p for some $\mathscr{D}_{2}$ such that $s_{2} \stackrel{a}{\longrightarrow} \mathscr{D}_{2}$.

Since $\phi^{\prime} \in \mathbb{F}_{\mathrm{PML}}^{i}$, by the induction hypothesis there exists $\mathscr{G} \in 2^{S / \sim_{\mathrm{PB}, \mathrm{gbg}, \sqcup}^{i}}$ such that $\bigcup_{C \in \mathscr{G}} C=\mathscr{M}_{\mathrm{PML}_{\forall, \leq}}\left[\left[\phi^{\prime}\right]\right]$. Then:

- $s_{1} \stackrel{a}{\longrightarrow}$ and $\underset{s_{1} \stackrel{\bigsqcup^{\longrightarrow}}{\longrightarrow} \mathscr{D}_{1}}{ } \mathscr{D}_{1}(\bigcup \mathscr{G}) \leq p$.

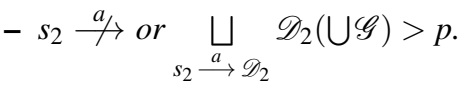

- In the $(\Longleftarrow)$ part, if $s_{1} \sim_{\mathrm{PB}, \mathrm{gbg}, \sqcup}^{i} s_{2}$, then there exist $p \in \mathbb{R}_{[0,1]}$ and $\mathscr{G} \in 2^{S / \sim_{\mathrm{PB}, \mathrm{gbg}, \sqcup}^{i}}$ such that:

$$
\begin{aligned}
& \text { - } s_{1} \stackrel{a}{\longrightarrow} \text { and } \bigsqcup_{s_{1} \stackrel{a}{\longrightarrow} \mathscr{D}_{1}} \mathscr{D}_{1}(\bigcup \mathscr{G})=p \text {. } \\
& \text { - } s_{2} \stackrel{a}{\rightarrow} \text { or } \bigsqcup_{s_{2} \stackrel{\bigsqcup^{\prime}}{\longrightarrow} \mathscr{D}_{2}} \mathscr{D}_{2}(\bigcup \mathscr{G})=q \neq p
\end{aligned}
$$

Let $\mathscr{G}_{1}=\left\{C \in S / \sim_{\mathrm{PB}, \mathrm{gbg}, \sqcup}^{i} \mid \exists \mathscr{D}_{1} . s_{1} \stackrel{a}{\longrightarrow} \mathscr{D}_{1} \wedge \mathscr{D}_{1}(C)>0\right\}$ and $\mathscr{G}_{2}=\left\{C \in S / \sim_{\mathrm{PB}, \mathrm{gbg}, \sqcup}^{i}\right.$ $\left.\mid \exists \mathscr{D}_{2} \cdot s_{2} \stackrel{a}{\longrightarrow} \mathscr{D}_{2} \wedge \mathscr{D}_{2}(C)>0\right\}$. The distinguishing formula in $\mathbb{F}_{\mathrm{PML}_{\forall, \leq}^{i+1}}^{i}$ for $s_{1}$ and $s_{2}$ is then:

$$
\begin{aligned}
& -\langle a\rangle_{p} \phi_{\mathscr{G}} \text { if } s_{2} \stackrel{a}{\rightarrow} \text { or } p<q . \\
& -\langle a\rangle_{q} \phi_{\mathscr{G}} \text { if } s_{2} \stackrel{a}{\longrightarrow} \text { and } q<p .
\end{aligned}
$$

The proof of the second result is similar to the proof of the first one up to the use of $\Pi$ in place of $\sqcup, \geq$ in place of $\leq,<$ in place of $>$, and $>$ in place of $<$ wherever necessary.

\subsection{Multistep Variants of Probabilistic Bisimilarities and PCTL/PCTL*}

Bisimulation equivalences can also be defined by matching entire computations instead of individual transitions. A computation of an NPLTS is a sequence of action-labeled stateto-state steps, each of which is derived from a state-to-distribution transition by considering only the states in the support of the target distribution. The multistep variants of probabilistic bisimilarities for NPLTS models can be defined in several different ways.

The first option, inspired by bisimilarity for fully nondeterministic processes, consists of changing the one-step definitions by considering traces $\alpha \in A^{*}$ in place of actions $a \in A$ and $\stackrel{\alpha}{\Longrightarrow}$ in place of $\stackrel{a}{\longrightarrow}$ (resp. $\stackrel{\alpha}{\Longrightarrow}$ c in place of $\stackrel{a}{\longrightarrow}$ c), where $s \stackrel{\alpha}{\Longrightarrow} \mathscr{D}$ means that there 

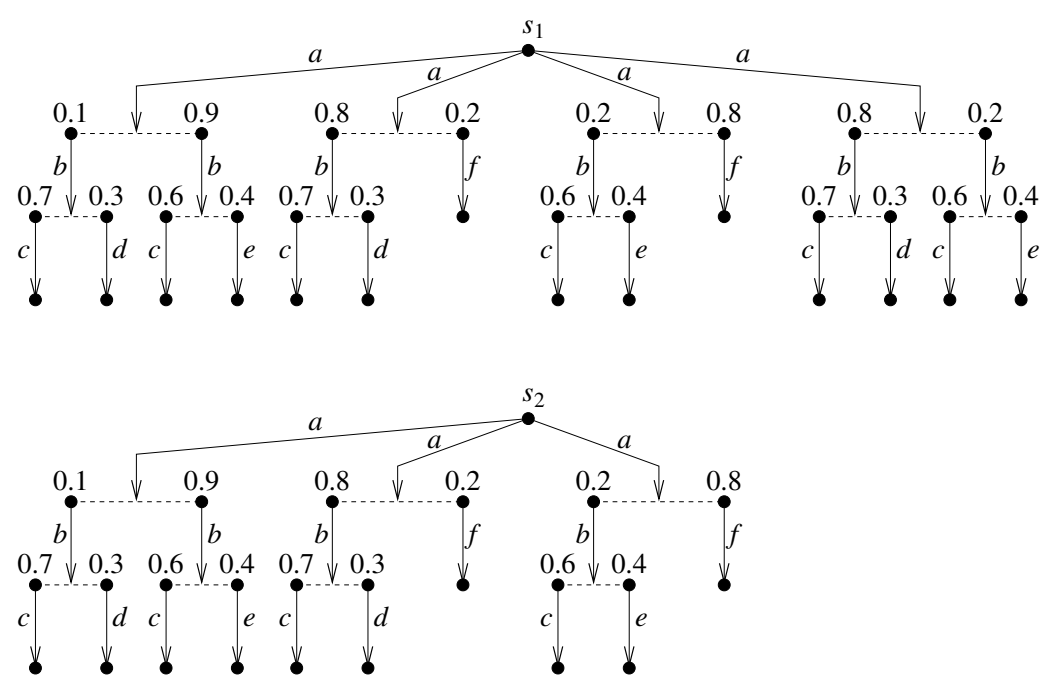

Fig. 4 Two models identified by $\sim_{\mathrm{PB}, \mathrm{gbg},=}$ and $\sim_{\mathrm{PB}, \mathrm{gbg}, \leq}$ that are distinguished by PCTL*

exists a computation from $s$ labeled with $\alpha$ whose last step is originated by a transition reaching distribution $\mathscr{D}$. When $\alpha$ is the empty sequence $\varepsilon$, we let $s \stackrel{\varepsilon}{\Longrightarrow} \delta_{s}$. It was shown in [26] that the discriminating power of bisimilarity for fully nondeterministic processes does not change if the multistep transition relation $\stackrel{\alpha}{\Longrightarrow}$ is used instead of the one-step relation $\stackrel{a}{\longrightarrow}$. As expected, this result carries over class-distribution and group-by-group probabilistic bisimilarities for nondeterministic and probabilistic processes (see App. A).

The second option, inspired by probabilistic bisimilarity for reactive probabilistic processes, does not only compare the probability arising from the last step of the computations, but additionally considers the probability of performing the entire computations, i.e., the product of the probabilities of the individual steps. While it can be shown that the discriminating power of the probabilistic bisimilarity for reactive probabilistic processes of [33] and of class-distribution probabilistic bisimilarities for nondeterministic and probabilistic processes does not change if multistep probability values are compared instead of one-step values, this is not the case with the group-by-group probabilistic bisimilarities (see App. B).

Finally, the third option, which is orthogonal to the previous two, consists of imposing some constraints along the computations, such as passing through specific sets of states at each step. This is the idea exploited in [42] in order to define - following the second option above - four probabilistic bisimilarities over nondeterministic and probabilistic Kripke structures that are respectively characterized by PCTL, PCTL*, and their variants without the next-time operator, which are interpreted as in [7].

It is worth noting that the strong 1-depth bisimulation of [42] and our $\sim_{\mathrm{PB}, \mathrm{gbg}, \leq}$ are deeply related. The main differences are that the former is defined over state-labeled models (nondeterministic and probabilistic Kripke structures) and considers generic binary relations, while the latter is defined over transition-labeled models (NPLTS models) and considers only equivalence relations. However, the probabilistic bisimilarities built in [42] on top of the strong 1-depth bisimulation are finer than our group-by-group probabilistic bisimilarities. Consider for instance the two NPLTS models in Fig. 4 . We have that $s_{1} \sim_{\mathrm{PB}, \mathrm{gbg},=} s_{2}$ - and hence $s_{1} \sim \sim_{\mathrm{PB}, \mathrm{gbg}, \leq s_{2}}$ - as witnessed by the equivalence relation that pairs states with identically labeled transitions and, in the case of $b$-transitions, identical target distributions. 
However, $s_{1}$ and $s_{2}$ are distinguished by the probabilistic bisimilarity of [42] that is characterized by PCTL*. Following [15], we view the two NPLTS models as two nondeterministic and probabilistic Kripke structures by eliminating actions from transitions and labeling each state with the set of its next-actions. Then the PCTL* formula $\operatorname{Pr}_{\leq 0.61}(\mathbf{X X} c)$ is satisfied by $s_{2}$ but it is not satisfied by $s_{1}$, because the probability of reaching in two steps a state that enables $c$ in the maximal resolution of $s_{1}$ starting with the rightmost $a$-transition is $0.8 \cdot 0.7+0.2 \cdot 0.6=0.68$ and hence it is greater than 0.61 .

\section{The Spectrum of Probabilistic Bisimilarities and PML Equivalences}

If we investigate the discriminating power of the six group-by-group probabilistic bisimilarities considered so far, we discover that all of them are coarser than $\sim_{\text {PB dis }}$ and, most importantly, five of them are coarser than $\sim_{\mathrm{PB} \text {,gbg, }=}$ and boil down to the same relation. The latter property consequently extends to the corresponding logical equivalences, with $\sim_{\mathrm{PML}_{\exists, \mathrm{I}}}$ turning out to be finer than the other five PML-based equivalences.

Theorem 5 Let $\mathscr{L}=(S, A, \longrightarrow)$ be an NPLTS and $s_{1}, s_{2} \in S$. Then:

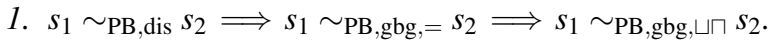

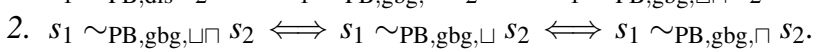

3. $s_{1} \sim \mathrm{PB}, \mathrm{gbg}, \sqcup s_{2} \Longleftrightarrow s_{1} \sim{ }_{\mathrm{PB}, \mathrm{gbg}, \leq s_{2}}$ when $\mathscr{L}$ is image finite.

4. $s_{1} \sim \mathrm{PB}, \mathrm{gbg}, \sqcap s_{2} \Longleftrightarrow s_{1} \sim{ }_{\mathrm{PB}, \mathrm{gbg}, \geq s_{2}}$ when $\mathscr{L}$ is image finite.

Proof We proceed as follows:

1. The fact that $s_{1} \sim$ PB, dis $s_{2}$ implies $s_{1} \sim$ PB,gbg, $=s_{2}$ is a straightforward consequence of the fact that a class-distribution probabilistic bisimulation is trivially $a=$-group-by-group probabilistic bisimulation.

Suppose now that $s_{1} \sim_{\mathrm{PB}, \mathrm{gbg},=} s_{2}$. This means that there exists $a=$-group-by-group probabilistic bisimulation $\mathscr{B}$ over $S$ such that $\left(s_{1}, s_{2}\right) \in \mathscr{B}$. In other words, whenever $\left(s_{1}^{\prime}, s_{2}^{\prime}\right) \in \mathscr{B}$, then for all $a \in A$ and $\mathscr{G} \in 2^{S / \mathscr{B}}$ :

- For each $s_{1}^{\prime} \stackrel{a}{\longrightarrow} \mathscr{D}_{1}$ there exists $s_{2}^{\prime} \stackrel{a}{\longrightarrow} \mathscr{D}_{2}$ such that $\mathscr{D}_{1}(\bigcup \mathscr{G})=\mathscr{D}_{2}(\bigcup \mathscr{G})$.

- For each $s_{2}^{\prime} \stackrel{a}{\longrightarrow} \mathscr{D}_{2}$ there exists $s_{1}^{\prime} \stackrel{a}{\longrightarrow} \mathscr{D}_{1}$ such that $\mathscr{D}_{2}(\bigcup \mathscr{G})=\mathscr{D}_{1}(\bigcup \mathscr{G})$.

This means that, whenever $\left(s_{1}^{\prime}, s_{2}^{\prime}\right) \in \mathscr{B}$, then for all $a \in A$ and $\mathscr{G} \in 2^{S / \mathscr{B}}$ :

- If $s_{1}^{\prime} \stackrel{a}{\longrightarrow}$, then $s_{2}^{\prime} \stackrel{a}{\longrightarrow}$ with $\underset{s_{1}^{\prime} \stackrel{a}{\longrightarrow} \mathscr{D}_{1}}{\bigcup}\left\{\mathscr{D}_{1}(\bigcup \mathscr{G})\right\} \subseteq \underset{s_{2}^{\prime} \stackrel{a}{\longrightarrow} \mathscr{D}_{2}}{\bigcup}\left\{\mathscr{D}_{2}(\bigcup \mathscr{G})\right\}$.

- If $s_{2}^{\prime} \stackrel{a}{\longrightarrow}$, then $s_{1}^{\prime} \stackrel{a}{\longrightarrow}$ with $\underset{s_{2}^{\prime} \stackrel{a}{\longrightarrow} \mathscr{D}_{2}}{\bigcup}\left\{\mathscr{D}_{2}(\bigcup \mathscr{G})\right\} \subseteq \underset{s_{1}^{\prime} \stackrel{a}{\longrightarrow} \mathscr{D}_{1}}{\bigcup}\left\{\mathscr{D}_{1}(\bigcup \mathscr{G})\right\}$.

Equivalently, if both $s_{1}^{\prime}$ and $s_{2}^{\prime}$ have at least one outgoing a-transition, then:

and hence:

$$
\underset{s_{1}^{\prime} \stackrel{a}{\longrightarrow} \mathscr{D}_{1}}{\bigcup}\left\{\mathscr{D}_{1}(\bigcup \mathscr{G})\right\}=\underset{s_{2}^{\prime} \stackrel{a}{\longrightarrow} \mathscr{D}_{2}}{\bigcup}\left\{\mathscr{D}_{2}(\bigcup \mathscr{G})\right\}
$$

$$
\begin{aligned}
\bigsqcup_{1}^{\prime} \stackrel{a}{\longrightarrow} \mathscr{D}_{1}(\bigcup \mathscr{G}) & =\underset{s_{2}^{\prime} \stackrel{\bigsqcup_{a}}{\longrightarrow} \mathscr{D}_{2}}{\mathscr{D}_{2}(\bigcup \mathscr{G})} \\
\prod_{a} \mathscr{D}_{1}(\bigcup \mathscr{G}) & =\prod_{\mathscr{D}_{2}(\bigcup \mathscr{G})}
\end{aligned}
$$

Thus, $\mathscr{B}$ is also a $\sqcup \sqcap$-group-by-group probabilistic bisimulation, i.e., $s_{1} \sim \mathrm{PB}, \mathrm{gbg}, \sqcup \sqcap s_{2}$. 


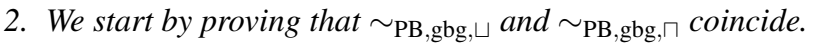

Suppose that $s_{1} \sim \mathrm{PB}, \mathrm{gbg}, \sqcup s_{2}$. This means that there exists a $\sqcup$-group-by-group probabilistic bisimulation $\mathscr{B}$ over $S$ such that $\left(s_{1}, s_{2}\right) \in \mathscr{B}$. In other words, whenever $\left(s_{1}^{\prime}, s_{2}^{\prime}\right) \in$ $\mathscr{B}$, then for all $a \in A$ and $\mathscr{G} \in 2^{S / \mathscr{B}}$ it holds that $s_{1}^{\prime} \stackrel{a}{\longrightarrow}$ iff $s_{2}^{\prime} \stackrel{a}{\longrightarrow}$ and:

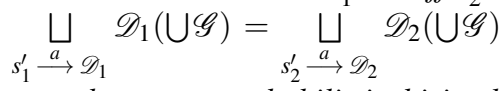

Then $\mathscr{B}$ must be a $\sqcap$-group-by-group probabilistic bisimulation as well and hence $s_{1} \sim \mathrm{PB}, \mathrm{gbg}, \sqcap s_{2}$. In fact, if this were not the case, then there would exist $a^{\prime} \in A$ and $\mathscr{G}^{\prime} \in 2^{S / \mathscr{B}}$ such that $s_{1}^{\prime} \stackrel{a^{\prime}}{\longrightarrow}, s_{2}^{\prime} \stackrel{a^{\prime}}{\longrightarrow}$, and:

$$
\prod_{s_{1}^{\prime} \stackrel{a^{\prime}}{\longrightarrow} \mathscr{D}_{1}} \mathscr{D}_{1}\left(\bigcup \mathscr{G}^{\prime}\right) \neq \prod_{s_{2}^{\prime} \stackrel{a^{\prime}}{\longrightarrow} \mathscr{D}_{2}} \mathscr{D}_{2}\left(\bigcup \mathscr{G}^{\prime}\right)
$$

As a consequence, denoting by $\mathscr{G}^{\prime \prime}$ the group of all the equivalence classes not in $\mathscr{G}^{\prime}$, it would hold that $s_{1}^{\prime} \stackrel{a^{\prime}}{\longrightarrow}, s_{2}^{\prime} \stackrel{a^{\prime}}{\longrightarrow}$, and:

$$
\begin{aligned}
\underset{s_{1}^{\prime} \stackrel{a^{\prime}}{\longrightarrow} \mathscr{D}_{1}}{\bigsqcup} \mathscr{D}_{1}\left(\bigcup \mathscr{G}^{\prime \prime}\right) & =1-\prod_{s_{1}^{\prime}} \mathscr{a}_{s_{2}^{\prime}}^{\longrightarrow} \mathscr{D}_{1}\left(\bigcup \mathscr{G}^{\prime}\right) \neq \\
& \neq 1-\prod_{s_{2}^{\prime}}^{\stackrel{a^{\prime}}{\longrightarrow} \mathscr{D}_{2}} \mathscr{D}_{2}\left(\bigcup \mathscr{G}^{\prime}\right)=\underset{s_{2}^{\prime} \stackrel{a^{\prime}}{\longrightarrow} \mathscr{D}_{2}}{\bigsqcup} \mathscr{D}_{2}\left(\bigcup \mathscr{G}^{\prime \prime}\right)
\end{aligned}
$$

thus contradicting the fact that $\mathscr{B}$ is $a \sqcup$-group-by-group probabilistic bisimulation.

By proceeding in a similar way, we can prove that $s_{1} \sim \mathrm{PB}, \mathrm{gbg}, \sqcap s_{2}$ implies $s_{1} \sim \mathrm{PB}, \mathrm{gbg}, \sqcup s_{2}$. We conclude by proving that $\sim_{\mathrm{PB}, \mathrm{gbg}, \sqcup \sqcap}$ and $\sim_{\mathrm{PB}, \mathrm{gbg}, \sqcup \text { coincide. }}$

If $s_{1} \sim \sim_{\mathrm{PB}, \mathrm{gbg}, \sqcup \sqcap} s_{2}$, then $s_{1} \sim \sim_{\mathrm{PB}, \mathrm{gbg}, \sqcup s_{2}}$ because $a \sqcup \sqcap$-group-by-group probabilistic bisimulation is trivially a $\sqcup$-group-by-group probabilistic bisimulation. Suppose now that $s_{1} \sim \mathrm{PB}, \mathrm{gbg}, \sqcup s_{2}$. This means that there exists a $\sqcup$-group-by-group probabilistic bisimulation $\mathscr{B}$ over $S$ such that $\left(s_{1}, s_{2}\right) \in \mathscr{B}$. Since $\mathscr{B}$ must also be a $\sqcap$-group-by-group probabilistic bisimulation, whenever $\left(s_{1}^{\prime}, s_{2}^{\prime}\right) \in \mathscr{B}$, then for all $a \in A$ and $\mathscr{G} \in 2^{S / \mathscr{B}}$ it holds that $s_{1}^{\prime} \stackrel{a}{\longrightarrow}$ iff $s_{2}^{\prime} \stackrel{a}{\longrightarrow}$ and:

$$
\begin{aligned}
& \bigsqcup_{s_{1}^{\prime} \stackrel{a^{\longrightarrow}}{\longrightarrow} \mathscr{D}_{1}} \mathscr{D}_{1}(\bigcup \mathscr{G})=\bigsqcup_{s_{2}^{\prime} \stackrel{a^{\longrightarrow}}{\longrightarrow} \mathscr{D}_{2}} \mathscr{D}_{2}(\bigcup \mathscr{G}) \\
& \prod_{s_{1}^{\prime} \stackrel{a}{\longrightarrow} \mathscr{D}_{1}} \mathscr{D}_{1}(\bigcup \mathscr{G})=\prod_{s_{2}^{\prime} \stackrel{a}{\longrightarrow} \mathscr{D}_{2}} \mathscr{D}_{2}(\bigcup \mathscr{G})
\end{aligned}
$$

This means that $\mathscr{B}$ is also a $\sqcup П$-group-by-group probabilistic bisimulation, i.e., $s_{1} \sim$ PB,gbg, $\sqcap s_{2}$.

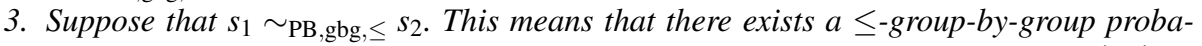
bilistic bisimulation $\mathscr{B}$ over $S$ such that $\left(s_{1}, s_{2}\right) \in \mathscr{B}$. In other words, whenever $\left(s_{1}^{\prime}, s_{2}^{\prime}\right) \in$ $\mathscr{B}$, then for all $a \in A$ and $\mathscr{G} \in 2^{S / \mathscr{B}}$ :

- For each $s_{1}^{\prime} \stackrel{a}{\longrightarrow} \mathscr{D}_{1}$ there exists $s_{2}^{\prime} \stackrel{a}{\longrightarrow} \mathscr{D}_{2}$ such that $\mathscr{D}_{1}(\bigcup \mathscr{G}) \leq \mathscr{D}_{2}(\bigcup \mathscr{G})$.

- For each $s_{2}^{\prime} \stackrel{a}{\longrightarrow} \mathscr{D}_{2}$ there exists $s_{1}^{\prime} \stackrel{a}{\longrightarrow} \mathscr{D}_{1}$ such that $\mathscr{D}_{2}(\bigcup \mathscr{G}) \leq \mathscr{D}_{1}(\bigcup \mathscr{G})$.

This means that, whenever $\left(s_{1}^{\prime}, s_{2}^{\prime}\right) \in \mathscr{B}$, then for all $a \in A$ and $\mathscr{G} \in 2^{S / \mathscr{B}}$ :

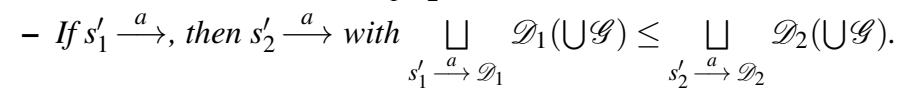

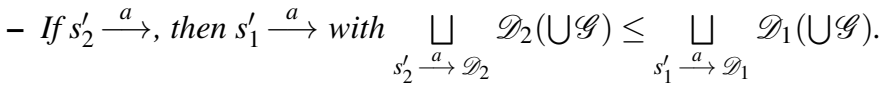

Equivalently, if both $s_{1}^{\prime}$ and $s_{2}^{\prime}$ have at least one outgoing a-transition, then:

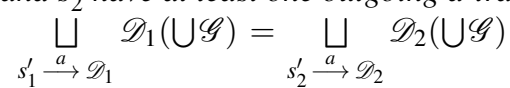

Thus, $\mathscr{B}$ is also a $\sqcup$-group-by-group probabilistic bisimulation, i.e., $s_{1} \sim \mathrm{PB}, \mathrm{gbg}, \sqcup s_{2}$.

The reverse implication holds too when $\mathscr{L}$ is image finite. In fact, this property guaran- 
tees that the following two sets:

$$
\bigcup_{s_{1}^{\prime} \stackrel{a}{\longrightarrow} \mathscr{D}_{1}}\left\{\mathscr{D}_{1}(\bigcup \mathscr{G})\right\} \quad \text { and } \underset{s_{2}^{\prime} \stackrel{a}{\longrightarrow} \mathscr{D}_{2}}{\bigcup}\left\{\mathscr{D}_{2}(\bigcup \mathscr{G})\right\}
$$

are finite. In turn, the finiteness of those two sets ensures that their suprema respectively belong to the two sets themselves. As a consequence, starting from:

or equivalently:

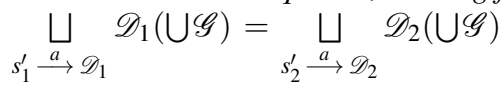

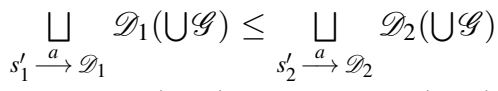

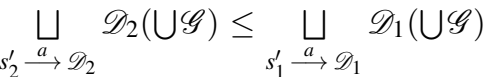

when both $s_{1}^{\prime}$ and $s_{2}^{\prime}$ have at least one outgoing a-transition, the following holds:

- For each $s_{1}^{\prime} \stackrel{a}{\longrightarrow} \mathscr{D}_{1}^{\prime}$ there exists $s_{2}^{\prime} \stackrel{a}{\longrightarrow} \mathscr{D}_{2}^{\prime}$ such that $\mathscr{D}_{1}^{\prime}(\cup \mathscr{G}) \leq \mathscr{D}_{2}^{\prime}(\bigcup \mathscr{G})$, because

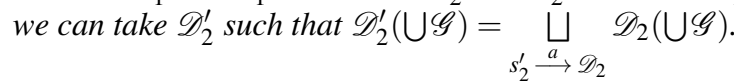

- For each $s_{2}^{\prime} \stackrel{a}{\longrightarrow} \mathscr{D}_{2}^{\prime}$ there exists $s_{1}^{\prime} \stackrel{a}{\longrightarrow} \mathscr{D}_{1}^{\prime}$ such that $\mathscr{D}_{2}^{\prime}(\bigcup \mathscr{G}) \leq \mathscr{D}_{1}^{\prime}(\bigcup \mathscr{G})$, because

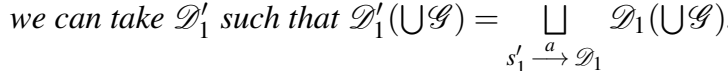

4. Similar to the proof of the previous result up to the use of $\geq$ in place of $\leq$ and $\sqcap$ in place of $\sqcup$ wherever necessary.

The two implications in Thm. 5 cannot be reversed: Fig. 1 shows that $\sim_{\mathrm{PB} \text {,dis }}$ is strictly

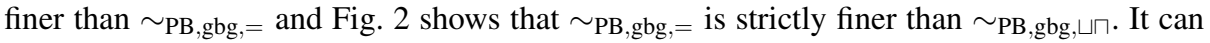
be noted from its proof that the result relating $\sim_{\mathrm{PB}, \mathrm{gbg}, \sqcup \sqcap,} \sim_{\mathrm{PB}, \mathrm{gbg}, \sqcup,}$, and $\sim_{\mathrm{PB}, \mathrm{gbg}, \sqcap}$ holds because groups of equivalence classes are considered. Analogous bisimilarities defined in a class-by-class fashion would not coincide.

Another interesting property is that the five coinciding group-by-group probabilistic bisimilarities are the same as their ct-variants, and hence are insensitive to whether deterministic or randomized schedulers are employed to resolve nondeterminism. This is not the case with $\sim_{\mathrm{PB} \text {,dis }}$ and $\sim_{\mathrm{PB} \text {,gbg. }}$. . Moreover, the ct-variants of all the six group-by-group probabilistic bisimilarities boil down to the same equivalence, which is $\sim_{\mathrm{PB}, \mathrm{gbg}, \leq}$, meaning that, in the bisimulation game, randomized schedulers reduce the discriminating power of the $=$-comparison to that of the $\leq$-comparison.

Theorem 6 Let $\mathscr{L}=(S, A, \longrightarrow)$ be an NPLTS and $s_{1}, s_{2} \in S$. Then:

1. $s_{1} \sim{ }_{\mathrm{PB}, \mathrm{dis}}^{\mathrm{ct}} s_{2} \Longrightarrow s_{1} \sim_{\mathrm{PB}, \mathrm{gbg},=}^{\mathrm{ct}} s_{2}$.

2. $s_{1} \sim \sim_{\mathrm{PB}, \mathrm{gbg}, \triangleright} s_{2} \Longrightarrow s_{1} \sim_{\mathrm{PB}, \mathrm{gbg}, \triangleright}^{\mathrm{ct}} s_{2}$ for $\triangleright \in\{=, \leq, \geq, \sqcup \sqcap, \sqcup, \sqcap\}$.

3. $s_{1} \sim \sim_{\mathrm{PB}, \mathrm{gbg}, \triangleleft} s_{2} \Longleftarrow s_{1} \sim_{\mathrm{PB}, \mathrm{gbg}, \triangleleft}^{\mathrm{ct}} s_{2}$ for $\triangleleft \in\{\leq, \geq\}$.

4. $s_{1} \sim \sim_{\mathrm{PB}, \mathrm{gbg}, \triangleleft} s_{2} \Longleftarrow s_{1} \sim_{\mathrm{PB}, \mathrm{gbg}, \triangleleft}^{\mathrm{ct}} s_{2}$ for $\triangleleft \in\{\sqcup \sqcap, \sqcup, \sqcap\}$ when $\mathscr{L}$ is image finite.

5. $s_{1} \sim_{\mathrm{PB}, \mathrm{gbg},=}^{\mathrm{ct}} s_{2} \Longleftrightarrow s_{1} \sim_{\mathrm{PB}, \mathrm{gbg}, \sqcup \sqcap}^{\mathrm{ct}} s_{2}$ when $\mathscr{L}$ is image finite.

Proof We proceed as follows:

1. A straightforward consequence of the fact that a class-distribution ct-probabilistic bisimulation is trivially $a=$-group-by-group ct-probabilistic bisimulation.

2. An immediate consequence of the fact that an ordinary transition is a special case of combined transition in which a single transition is taken with probability 1. 
3. Suppose that $s_{1} \sim_{\mathrm{PB}, \mathrm{gbg}, \leq}^{\mathrm{ct}} s_{2}$. This means that there exists a $\leq$-group-by-group ct-probabilistic bisimulation $\mathscr{B}$ over $S$ such that $\left(s_{1}, s_{2}\right) \in \mathscr{B}$. In other words, whenever $\left(s_{1}^{\prime}, s_{2}^{\prime}\right) \in \mathscr{B}$, then for all $a \in A$ and $\mathscr{G} \in 2^{S / \mathscr{B}}$ it holds that for each $s_{1}^{\prime} \stackrel{a}{\longrightarrow} \mathscr{D}_{1}$ there exists $s_{2}^{\prime} \stackrel{a}{\longrightarrow} \mathscr{D}_{2}$ such that $\mathscr{D}_{1}(\bigcup \mathscr{G}) \leq \mathscr{D}_{2}(\bigcup \mathscr{G})$. On the side of $s_{2}^{\prime}$, this means that there exist $n \in \mathbb{N}_{>0},\left(p_{i} \in \mathbb{R}_{0,1]} \mid 1 \leq i \leq n\right)$, and $\left(s_{2}^{\prime} \stackrel{a}{\longrightarrow} \mathscr{D}_{2, i} \mid 1 \leq i \leq n\right)$ such that $\sum_{i=1}^{n} p_{i}=1$ and $\sum_{i=1}^{n} p_{i} \cdot \mathscr{D}_{2, i}=\mathscr{D}_{2}$. As a consequence:

$$
\mathscr{D}_{2}(\bigcup \mathscr{G}) \leq \sum_{i=1}^{n} p_{i} \cdot \max _{1 \leq i \leq n} \mathscr{D}_{2, i}(\bigcup \mathscr{G})=\max _{1 \leq i \leq n} \mathscr{D}_{2, i}(\bigcup \mathscr{G}) \cdot \sum_{i=1}^{n} p_{i}=\max _{1 \leq i \leq n} \mathscr{D}_{2, i}(\bigcup \mathscr{G})
$$

and hence there exists $\mathscr{D}_{2}^{\prime}$ such that $s_{2}^{\prime} \stackrel{a}{\longrightarrow} \mathscr{D}_{2}^{\prime}$ with $\mathscr{D}_{1}(\bigcup \mathscr{G}) \leq \mathscr{D}_{2}^{\prime}(\bigcup \mathscr{G})$. This means that $\mathscr{B}$ is also a $\leq$-group-by-group probabilistic bisimulation, i.e., $s_{1} \sim \mathrm{PB}, \mathrm{gbg}, \leq s_{2}$.

The proof of $s_{1} \sim_{\mathrm{PB}, \mathrm{gbg}, \geq}^{\mathrm{ct}} s_{2} \Longrightarrow s_{1} \sim \mathrm{PB}, \mathrm{gbg}, \geq s_{2}$ is similar to the previous proof up to the use of $\geq$ in place of $\leq$ and $\min$ in place of $\max$ wherever necessary.

4. Suppose that $s_{1} \sim_{\mathrm{PB}, \mathrm{gbg}, \sqcup \sqcap}^{\mathrm{ct}} s_{2}$. This means that there exists a பП-group-by-group ct-probabilistic bisimulation $\mathscr{B}$ over $S$ such that $\left(s_{1}, s_{2}\right) \in \mathscr{B}$. In other words, whenever $\left(s_{1}^{\prime}, s_{2}^{\prime}\right) \in \mathscr{B}$, then for all $a \in A$ and $\mathscr{G} \in 2^{S / \mathscr{B}}$ it holds that $s_{1}^{\prime} \stackrel{a}{\longrightarrow}$ iff $s_{2}^{\prime} \stackrel{a}{\longrightarrow}$ and:

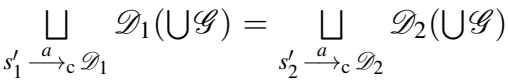

$$
\begin{aligned}
& \prod_{s_{1}^{\prime} \stackrel{a}{\longrightarrow} \mathrm{c} \mathscr{D}_{1}} \mathscr{D}_{1}(\bigcup \mathscr{G})=\prod_{s_{2}^{\prime} \stackrel{a}{\longrightarrow} \underset{\mathrm{c}}{s_{2}} \mathscr{D}_{2}} \mathscr{D}_{2}(\bigcup \mathscr{G})
\end{aligned}
$$

Given a $\in A, \mathscr{G} \in 2^{S / \mathscr{B}}$, and $s \in S$ having at least one outgoing a-transition, when $\mathscr{L}$ is image finite it holds that:

$$
\begin{aligned}
& \bigsqcup_{s \stackrel{a}{\longrightarrow} \mathrm{d}} \mathscr{D}(\bigcup \mathscr{G})=\bigsqcup_{s \stackrel{a}{\longrightarrow} \mathscr{D}} \mathscr{D}(\bigcup \mathscr{G}) \\
& \prod_{s \stackrel{a}{\longrightarrow} \mathfrak{c} \mathscr{D}} \mathscr{D}(\bigcup \mathscr{G})=\prod_{s \stackrel{a}{\longrightarrow} \mathscr{D}} \mathscr{D}(\bigcup \mathscr{G})
\end{aligned}
$$

because the supremum and the infimum on the left are respectively achieved by two ordinary a-transitions of $s$. In fact, let $\mathscr{D} \sqcup$ (resp. $\mathscr{D}_{\sqcap}$ ) be the target of an a-transition of $s$ assigning the maximum (resp. minimum) value to $\bigcup \mathscr{G}$ among all the a-transitions of $s$ and consider an arbitrary convex combination of a tuple $\left(s \stackrel{a}{\longrightarrow} \mathscr{D}_{i} \mid 1 \leq i \leq n\right)$ of those transitions, with probabilities $p_{1}, \ldots, p_{n}$ and $n \in \mathbb{N}_{>0}$. Then:

$$
\begin{aligned}
& \sum_{i=1}^{n} p_{i} \cdot \mathscr{D}_{i}(\bigcup \mathscr{G}) \leq \sum_{i=1}^{n} p_{i} \cdot \mathscr{D} \sqcup(\bigcup \mathscr{G})=\mathscr{D} \sqcup(\bigcup \mathscr{G}) \\
& \sum_{i=1}^{n} p_{i} \cdot \mathscr{D}_{i}(\bigcup \mathscr{G}) \geq \sum_{i=1}^{n} p_{i} \cdot \mathscr{D}_{\sqcap}(\bigcup \mathscr{G})=\mathscr{D}_{\sqcap}(\bigcup \mathscr{G})
\end{aligned}
$$

As a consequence, whenever $\left(s_{1}^{\prime}, s_{2}^{\prime}\right) \in \mathscr{B}$, then for all $a \in A$ and $\mathscr{G} \in 2^{S / \mathscr{B}}$ it holds that $s_{1}^{\prime} \stackrel{a}{\longrightarrow}$ iff $s_{2}^{\prime} \stackrel{a}{\longrightarrow}$ and $:$

$$
\begin{aligned}
& \bigsqcup_{s_{1}^{\prime} \stackrel{\bigsqcup^{\longrightarrow}}{\longrightarrow} \mathscr{D}_{1}} \mathscr{D}_{1}(\bigcup \mathscr{G})=\bigsqcup_{s_{2}^{\prime} \stackrel{\bigsqcup_{\longrightarrow}}{\longrightarrow} \mathscr{D}_{2}} \mathscr{D}_{2}(\bigcup \mathscr{G}) \\
& \prod_{s_{1}^{\prime} \stackrel{a}{\longrightarrow} \mathscr{D}_{1}} \mathscr{D}_{1}(\bigcup \mathscr{G})=\prod_{s_{2}^{\prime} \stackrel{a}{\longrightarrow} \mathscr{D}_{2}} \mathscr{D}_{2}(\bigcup \mathscr{G})
\end{aligned}
$$

This means that $\mathscr{B}$ is also a பП-group-by-group probabilistic bisimulation, i.e., $s_{1} \sim \mathrm{PB}, \mathrm{gbg}, \sqcup \sqcap s_{2}$.

The proofs of $s_{1} \sim_{\mathrm{PB}, \mathrm{gbg}, \sqcup}^{\mathrm{ct}} s_{2} \Longrightarrow s_{1} \sim \mathrm{PB}, \mathrm{gbg}, \sqcup s_{2}$ and $s_{1} \sim_{\mathrm{PB}, \mathrm{gbg}, \sqcap}^{\mathrm{ct}} s_{2} \Longrightarrow s_{1} \sim \mathrm{PB}, \mathrm{gbg}, \sqcap s_{2}$ are similar to the previous proof and rely on $\mathscr{L}$ being image finite.

5. Suppose that $s_{1} \sim_{\mathrm{PB}, \mathrm{gbg},=}^{\mathrm{ct}} s_{2}$. This means that there exists $a=$-group-by-group ct-probabilistic bisimulation $\mathscr{B}$ over $S$ such that $\left(s_{1}, s_{2}\right) \in \mathscr{B}$. In other words, whenever $\left(s_{1}^{\prime}, s_{2}^{\prime}\right) \in \mathscr{B}$, then for all $a \in A$ and $\mathscr{G} \in 2^{S / \mathscr{B}}$ :

- For each $s_{1}^{\prime} \stackrel{a}{\longrightarrow} \mathscr{D}_{1}$ there exists $s_{2}^{\prime} \stackrel{a}{\longrightarrow} \mathrm{c} \mathscr{D}_{2}$ such that $\mathscr{D}_{1}(\bigcup \mathscr{G})=\mathscr{D}_{2}(\bigcup \mathscr{G})$. 
- For each $s_{2}^{\prime} \stackrel{a}{\longrightarrow} \mathscr{D}_{2}$ there exists $s_{1}^{\prime} \stackrel{a}{\longrightarrow}_{\mathrm{c}} \mathscr{D}_{1}$ such that $\mathscr{D}_{2}(\bigcup \mathscr{G})=\mathscr{D}_{1}(\bigcup \mathscr{G})$.

This implies that, whenever $\left(s_{1}^{\prime}, s_{2}^{\prime}\right) \in \mathscr{B}$, then for all $a \in A$ and $\mathscr{G} \in 2^{S / \mathscr{B}}$ :

- For each $s_{1}^{\prime} \stackrel{a}{\longrightarrow} \mathrm{c} \mathscr{D}_{1}$ there exists $s_{2}^{\prime} \stackrel{a}{\longrightarrow} \mathscr{D}_{2}$ such that $\mathscr{D}_{1}(\bigcup \mathscr{G})=\mathscr{D}_{2}(\bigcup \mathscr{G})$.

- For each $s_{2}^{\prime} \stackrel{a}{\longrightarrow} \mathscr{D}_{2}$ there exists $s_{1}^{\prime} \stackrel{a}{\longrightarrow}_{\mathrm{c}} \mathscr{D}_{1}$ such that $\mathscr{D}_{2}(\bigcup \mathscr{G})=\mathscr{D}_{1}(\bigcup \mathscr{G})$.

As a consequence, whenever $\left(s_{1}^{\prime}, s_{2}^{\prime}\right) \in \mathscr{B}$, then for all $a \in A$ and $\mathscr{G} \in 2^{S / \mathscr{B}}$ :

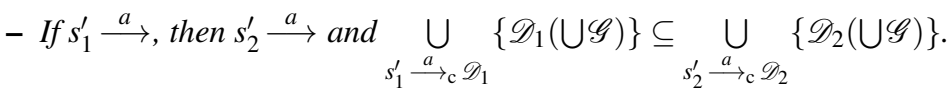

- If $s_{2}^{\prime} \stackrel{a}{\longrightarrow}$, then $s_{1}^{\prime} \stackrel{a}{\longrightarrow}$ and $\underset{s_{2}^{\prime} \underset{a_{\mathrm{c}}}{\longrightarrow} \mathscr{D}_{2}}{\bigcup}\left\{\mathscr{D}_{2}(\bigcup \mathscr{G})\right\} \subseteq \underset{s_{1}^{\prime} \underset{a_{\mathrm{c}}}{\longrightarrow} \mathscr{D}_{1}}{\bigcup}\left\{\mathscr{D}_{1}(\bigcup \mathscr{G})\right\}$.

Equivalently, if both $s_{1}^{\prime}$ and $s_{2}^{\prime}$ have at least one outgoing a-transition, then:

and hence:

$$
\bigcup_{a}\left\{\mathscr{D}_{1}(\bigcup \mathscr{G})\right\}=\underset{s_{2}^{\prime} \underset{\mathfrak{a}_{1}}{\longrightarrow} \bigcup_{\mathrm{c}} \mathscr{D}_{2}}{ }\left\{\mathscr{D}_{2}(\bigcup \mathscr{G})\right\}
$$

$$
\begin{aligned}
& \bigsqcup_{s_{1}^{\prime} \stackrel{a}{\rightarrow} \mathrm{c} \mathscr{D}_{1}} \mathscr{D}_{1}(\bigcup \mathscr{G})=\bigsqcup_{s_{2}^{\prime} \stackrel{a_{c}}{\longrightarrow} \mathscr{D}_{2}} \mathscr{D}_{2}(\bigcup \mathscr{G}) \\
& \prod_{s_{1}^{\prime} \stackrel{a}{\longrightarrow} \mathscr{D}_{1}} \mathscr{D}_{1}(\bigcup \mathscr{G})=\prod_{s_{2}^{\prime} \stackrel{a_{a}}{\longrightarrow} \mathscr{D}_{2}} \mathscr{D}_{2}(\bigcup \mathscr{G})
\end{aligned}
$$

Thus, $\mathscr{B}$ is also a $\sqcap$-group-by-group ct-probabilistic bisimulation, i.e., $s_{1} \sim_{\mathrm{PB}, \mathrm{gbg}, \sqcup \sqcap}^{\mathrm{ct}} s_{2}$. Suppose now that $s_{1} \sim_{\mathrm{PB}, \mathrm{gbg}, \sqcup \sqcap}^{\mathrm{ct}} s_{2}$. This means that there exists $a \sqcup \sqcap$-group-by-group ct-probabilistic bisimulation $\mathscr{B}$ over $S$ such that $\left(s_{1}, s_{2}\right) \in \mathscr{B}$. Given $a \in A$ and $\mathscr{G} \in 2^{S / \mathscr{B}}$, assume that there exists $s_{1} \stackrel{a}{\longrightarrow} \mathscr{D}_{1}$ such that $\mathscr{D}_{1}(\bigcup \mathscr{G})=p$. Since $\left(s_{1}, s_{2}\right) \in \mathscr{B}$ and $\mathscr{L}$ is image finite, there exist $s_{2} \stackrel{a}{\longrightarrow} \mathrm{c} \mathscr{D}_{2}^{\prime}$ such that $\mathscr{D}_{2}^{\prime}(\bigcup \mathscr{G})=p^{\prime} \leq p$ and $s_{2} \stackrel{a}{\longrightarrow} \mathscr{D}_{2}^{\prime \prime}$ such that $\mathscr{D}_{2}^{\prime \prime}(\bigcup \mathscr{G})=p^{\prime \prime} \geq p$. If $p^{\prime}=p\left(\right.$ resp. $\left.p^{\prime \prime}=p\right)$, then $s_{1} \stackrel{a}{\longrightarrow} \mathscr{D}_{1}$ is trivially matched by $s_{2} \stackrel{a}{\longrightarrow} \mathscr{D}_{2}^{\prime}$ (resp. $\left.s_{2} \stackrel{a}{\longrightarrow} \mathscr{D}_{2}^{\prime \prime}\right)$ with respect to $\sim_{\mathrm{PB}, \mathrm{gbg},=}^{\mathrm{ct}}$ when considering $\mathscr{G}$.

Assume that $p^{\prime}<p<p^{\prime \prime}$ and note that $s_{2} \stackrel{a}{\longrightarrow}\left(x \cdot \mathscr{D}_{2}^{\prime}+y \cdot \mathscr{D}_{2}^{\prime \prime}\right)$ for all $x, y \in \mathbb{R}_{0,1]}$ such that $x+y=1$. Indeed, directly from the definition of combined transition, we have that:

- Since $s_{2} \stackrel{a}{\longrightarrow}_{\mathrm{c}} \mathscr{D}_{2}^{\prime}$, there exist $n \in \mathbb{N}_{>0},\left(p_{i}^{\prime} \in \mathbb{R}_{0,1]} \mid 1 \leq i \leq n\right)$, and $\left(s_{2} \stackrel{a}{\longrightarrow} \hat{\mathscr{D}}_{i}^{\prime} \mid\right.$ $1 \leq i \leq n)$ such that $\sum_{i=1}^{n} p_{i}^{\prime}=1$ and $\sum_{i=1}^{n} p_{i}^{\prime} \cdot \hat{\mathscr{D}}_{i}^{\prime}=\mathscr{D}_{2}^{\prime}$.

- Since $s_{2} \stackrel{a}{\longrightarrow} \mathscr{D}_{2}^{\prime \prime}$, there exist $m \in \mathbb{N}_{>0},\left(p_{j}^{\prime \prime} \in \mathbb{R}_{j 0,1]} \mid 1 \leq j \leq m\right)$, and $\left(s_{2} \stackrel{a}{\longrightarrow} \hat{\mathscr{D}}_{j}^{\prime \prime} \mid\right.$ $1 \leq j \leq m)$ such that $\sum_{j=1}^{m} p_{j}^{\prime \prime}=1$ and $\sum_{j=1}^{m} p_{j}^{\prime \prime} \cdot \hat{\mathscr{D}}_{j}^{\prime \prime}=\mathscr{D}_{2}^{\prime \prime}$.

Hence, $\left(x \cdot \mathscr{D}_{2}^{\prime}+y \cdot \mathscr{D}_{2}^{\prime \prime}\right)$ can be obtained from the appropriate combination of the two transition tuples $\left(s_{2} \stackrel{a}{\longrightarrow} \hat{\mathscr{D}}_{i}^{\prime} \mid 1 \leq i \leq n\right)$ and $\left(s_{2} \stackrel{a}{\longrightarrow} \hat{\mathscr{D}}_{j}^{\prime \prime} \mid 1 \leq j \leq m\right)$ with coefficients $\left(x \cdot p_{i}^{\prime} \in \mathbb{R}_{0,1]} \mid 1 \leq i \leq n\right)$ and $\left(y \cdot p_{j}^{\prime \prime} \in \mathbb{R}_{0,1]} \mid 1 \leq j \leq m\right)$. If we take $x=\frac{p^{\prime \prime}-p}{p^{\prime \prime}-p^{\prime}}$ and $y=\frac{p-p^{\prime}}{p^{\prime \prime}-p^{\prime}}$, then $s_{2} \stackrel{a}{\longrightarrow}{ }_{\mathrm{c}}\left(\frac{p^{\prime \prime}-p}{p^{\prime \prime}-p^{\prime}} \cdot \mathscr{D}_{2}^{\prime}+\frac{p-p^{\prime}}{p^{\prime \prime}-p^{\prime}} \cdot \mathscr{D}_{2}^{\prime \prime}\right)$ with:

$$
\begin{aligned}
\left(\frac{p^{\prime \prime}-p}{p^{\prime \prime}-p^{\prime}} \cdot \mathscr{D}_{2}^{\prime}+\frac{p-p^{\prime}}{p^{\prime \prime}-p^{\prime}} \cdot \mathscr{D}_{2}^{\prime \prime}\right)(\bigcup \mathscr{G}) & =\frac{p^{\prime \prime}-p}{p^{\prime \prime}-p^{\prime}} \cdot \mathscr{D}_{2}^{\prime}(\bigcup \mathscr{G})+\frac{p-p^{\prime}}{p^{\prime \prime}-p^{\prime}} \cdot \mathscr{D}_{2}^{\prime \prime}(\bigcup \mathscr{G}) \\
& =\frac{p^{\prime \prime}-p}{p^{\prime \prime}-p^{\prime}} \cdot p^{\prime}+\frac{p-p^{\prime}}{p^{\prime \prime}-p^{\prime}} \cdot p^{\prime \prime} \\
& =\frac{p^{\prime} \cdot p^{\prime \prime}-p \cdot p^{\prime}+p \cdot p^{\prime \prime}-p^{\prime} \cdot p^{\prime \prime}}{p^{\prime \prime}-p^{\prime}} \\
& =p \cdot \frac{p^{\prime \prime}-p^{\prime}}{p^{\prime \prime}-p^{\prime}}=p=\mathscr{D}_{1}(\bigcup \mathscr{G})
\end{aligned}
$$

Due to the generality of $\left(s_{1}, s_{2}\right) \in \mathscr{B}, a \in A$, and $\mathscr{G} \in 2^{S / \mathscr{B}}$, it turns out that $\mathscr{B}$ is also a $=$-group-by-group ct-probabilistic bisimulation, i.e., $s_{1} \sim_{\mathrm{PB}, \mathrm{gbg},=}^{\mathrm{ct}} s_{2}$.

The inclusion of $\sim_{\mathrm{PB}, \mathrm{dis}}^{\mathrm{ct}}$ in $\sim_{\mathrm{PB}, \mathrm{gbg},=}^{\mathrm{ct}}$ is strict, as shown by Fig. 1. Moreover, Fig. 2 shows that the inclusion of $\sim_{\mathrm{PB}, \mathrm{gbg}}=$ in $\sim_{\mathrm{PB}, \mathrm{gbg},=}^{\mathrm{ct}}=$ is strict; the central offer-transition of $s_{1}$ can be 


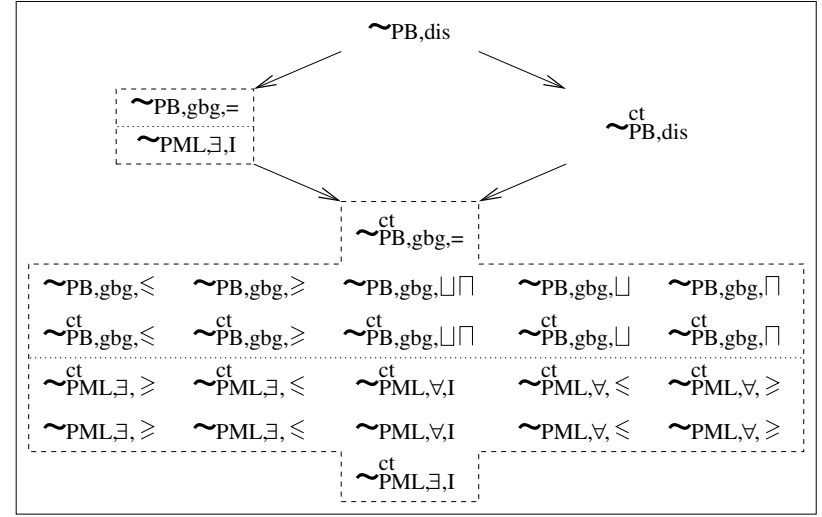

Fig. 5 The spectrum of probabilistic bisimilarities and PML-based equivalences

matched by a convex combination of the two offer-transitions of $s_{2}$ both with coefficient 0.5 .

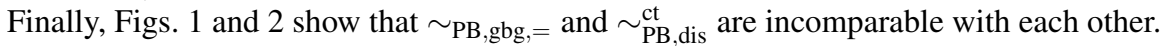

To summarize, all the relationships established so far among the probabilistic bisimilarities of this paper and those of [39], as well as the PML-based equivalences of this paper, are depicted in Fig. 5 in the case of image-finite NPLTS models satisfying the minimal probability assumption. Each arrow means more-discriminating-than. Equivalences collected in the same dashed box coincide; notice that the top part of each dashed box contains behavioral equivalences, while the bottom part contains logical ones.

It is possible to establish a deeper relationship among the four different probabilistic bisimilarities in Fig. 5. To this purpose, we introduce a parallel composition operator $\|_{L-}$ expressing that two NPLTS models synchronize on a set of actions $L$ and proceed independently of each other on any other action (we will apply the same notation to states).

Definition 10 Let $\mathscr{L}_{i}=\left(S_{i}, A, \longrightarrow_{i}\right)$ be an NPLTS for $i=1,2$. The parallel composition of $\mathscr{L}_{1}$ and $\mathscr{L}_{2}$ with synchronization on $L \subseteq A$ is the NPLTS $\mathscr{L}_{1} \|_{L} \mathscr{L}_{2}=\left(S_{1} \times S_{2}, A, \longrightarrow\right)$ where $\longrightarrow \subseteq\left(S_{1} \times S_{2}\right) \times A \times \operatorname{Distr}\left(S_{1} \times S_{2}\right)$ is such that $\left(s_{1}, s_{2}\right) \stackrel{a}{\longrightarrow} \mathscr{D}$ iff one of the following holds:

- $a \in L, s_{1} \stackrel{a}{\longrightarrow} \mathscr{D}_{1}, s_{2} \stackrel{a}{\longrightarrow} 2 \mathscr{D}_{2}$, and $\mathscr{D}\left(s_{1}^{\prime}, s_{2}^{\prime}\right)=\mathscr{D}_{1}\left(s_{1}^{\prime}\right) \cdot \mathscr{D}_{2}\left(s_{2}^{\prime}\right)$ for all $\left(s_{1}^{\prime}, s_{2}^{\prime}\right) \in S_{1} \times S_{2}$.

- $a \notin L, s_{1} \stackrel{a}{\longrightarrow} \mathscr{D}_{1}, \mathscr{D}\left(s_{1}^{\prime}, s_{2}^{\prime}\right)=\mathscr{D}_{1}\left(s_{1}^{\prime}\right)$ if $s_{2}^{\prime}=s_{2}$, and $\mathscr{D}\left(s_{1}^{\prime}, s_{2}^{\prime}\right)=0$ if $s_{2}^{\prime} \in S_{2} \backslash\left\{s_{2}\right\}$.

- $a \notin L, s_{2} \stackrel{a}{\longrightarrow} \mathscr{D}_{2}, \mathscr{D}\left(s_{1}^{\prime}, s_{2}^{\prime}\right)=\mathscr{D}_{2}\left(s_{2}^{\prime}\right)$ if $s_{1}^{\prime}=s_{1}$, and $\mathscr{D}\left(s_{1}^{\prime}, s_{2}^{\prime}\right)=0$ if $s_{1}^{\prime} \in S_{1} \backslash\left\{s_{1}\right\}$.

While $\sim_{\mathrm{PB} \text {, dis }}$ and $\sim_{\mathrm{PB} \text {,dis }}^{\mathrm{ct}}$ are congruences with respect to parallel composition [39], this is not the case for $\sim_{\mathrm{PB}, \mathrm{gbg},=}$ and $\sim_{\mathrm{PB}, \mathrm{gbg}, \leq}$. This is exemplified in Fig. 6. States $s_{1}$ and $s_{2}$ (which are distinguished by $\sim_{\mathrm{PB}, \text { dis }}$ ) are related by $\sim_{\mathrm{PB}, \mathrm{gbg},=}$ and $\sim_{\mathrm{PB}, \mathrm{gbg}, \leq}$, but $s_{1} \|_{A} s$ and $s_{2} \|_{A} s$ are no longer related by those two bisimilarities. For instance, the leftmost $a$-transition of $s_{1} \|_{A} s$ reaches with probability 0.46 the group of equivalence classes of states having only an outgoing transition labeled with $b$ or $c$, whilst the three $a$-transitions of $s_{2} \|_{A} s$ reach the same group with probabilities $0.54,0.28$, and 0.18 , respectively.

Interestingly, it turns out that $\sim_{\mathrm{PB} \text {,dis }}$ and $\sim_{\mathrm{PB} \text {,dis }}^{\mathrm{ct}}$ are the coarsest congruences, with respect to parallel composition, respectively contained in $\sim_{\mathrm{PB}, \mathrm{gbg},=}$ and $\sim_{\mathrm{PB}, \mathrm{gbg}, \leq}$.

Theorem 7 Let $\mathscr{L}=(S, A, \longrightarrow)$ be an image-finite NPLTS in which the target of each transition has finite support. Let $s_{1}, s_{2} \in S$. Then: 

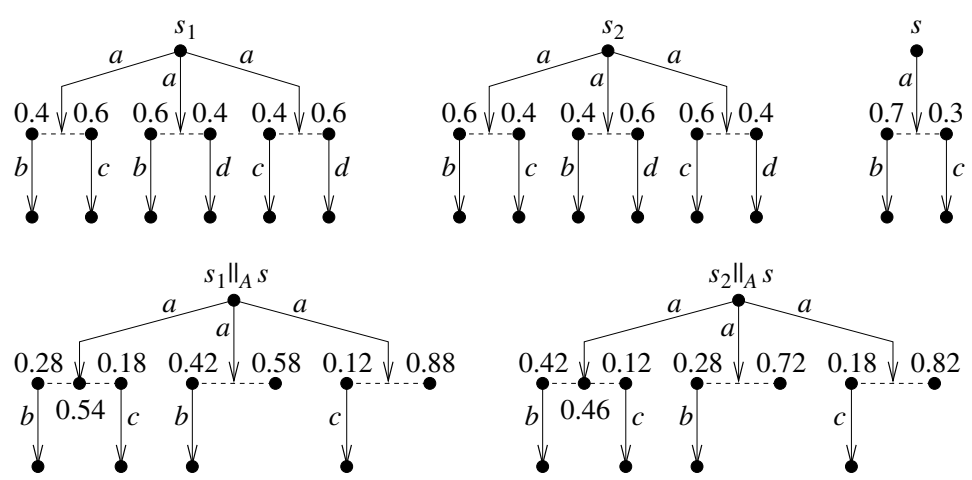

Fig. 6 Example illustrating that $\sim_{\mathrm{PB}, \mathrm{gbg},=}$ and $\sim_{\mathrm{PB}, \mathrm{gbg}, \leq}$ are not congruences

$$
\begin{aligned}
& s_{1} \sim_{\mathrm{PB}, \mathrm{dis}} s_{2} \Longleftrightarrow \forall L \subseteq A . \forall s \in S . s_{1}\left\|_{L} s \sim_{\mathrm{PB}, \mathrm{gbg},=s_{2}}\right\|_{L} s \\
& s_{1} \sim_{\mathrm{PB}, \text { dis }}^{\mathrm{ct}} s_{2} \Longleftrightarrow \forall L \subseteq A . \forall s \in S . s_{1} \|_{L} s \sim_{\mathrm{PB}, \mathrm{gbg}, \leq s_{2} \|_{L} s}
\end{aligned}
$$

Proof If $s_{1} \sim \mathrm{PB}$,dis $s_{2}$, then for all $L \subseteq A$ and $s \in S$ it holds that $s_{1}\left\|_{L} s \sim_{\mathrm{PB}, \mathrm{dis}} s_{2}\right\|_{L} s$ because $\sim_{\mathrm{PB} \text {,dis }}$ is a congruence with respect to parallel composition, and hence $s_{1} \|_{L} S \sim_{\mathrm{PB}, \mathrm{gbg},=}$ $s_{2} \|_{L} s$ by virtue of Thm. 5(1).

If $s_{1} \chi_{\mathrm{PB}, \mathrm{dis}} s_{2}$, we show that there exist $L \subseteq A$ and $s \in S$ such that $s_{1}\left\|_{L} s \chi_{\mathrm{PB}, \mathrm{gbg},=} s_{2}\right\|_{L} s$. We assume that $s_{1} \sim \mathrm{PB}, \mathrm{gbg},=s_{2}$, otherwise trivially $s_{1}\left\|_{\emptyset} \underline{0} \chi_{\mathrm{PB}, \mathrm{gbg},=} s_{2}\right\|_{\emptyset} \underline{0}$ where $\underline{0}$ is the terminal state.

From $s_{1} \chi_{\mathrm{PB}, \mathrm{dis}} s_{2}$, it follows that there exists a transition $s_{1} \stackrel{a}{\longrightarrow} \mathscr{D}_{1}$ such that for each transition $s_{2} \stackrel{a}{\longrightarrow} \mathscr{D}_{2}$ there exists an equivalence class $C \in S / \sim_{\mathrm{PB} \text {,dis }}$ such that $\mathscr{D}_{1}(C) \neq \mathscr{D}_{2}(C)$. Since $s_{1} \stackrel{a}{\longrightarrow} \mathscr{D}_{1}$ and $s_{1} \sim \mathrm{PB}, \mathrm{gbg},=s_{2}$, state $s_{2}$ has at least one outgoing a-transition; from the image finiteness of $\mathscr{L}$, the number of such transitions must be finite, say $m \in \mathbb{N}_{\geq 1}$.

Let us consider the following set of equivalence classes with respect to $\sim \mathrm{PB}, \mathrm{dis}$ :

$$
\mathscr{C}=\left\{C \in S / \sim \text { PB,dis } \mid \mathscr{D}_{1}(C)>0\right\}=\left\{C_{1}, C_{2}, \ldots, C_{n}\right\}
$$

which is not empty, because of the existence of $s_{1} \stackrel{a}{\longrightarrow} \mathscr{D}_{1}$, and finite, because $\operatorname{supp}\left(\mathscr{D}_{1}\right)$ is finite, hence $n \in \mathbb{N}_{\geq 1}$. Moreover, let $s$ be a state having a single outgoing transition $s \stackrel{a}{\longrightarrow} \mathscr{D}$, where $\mathscr{D}$ assigns probability $p_{i} \in \mathbb{R}_{0,1[}$ to the representative state $s_{C_{i}}$ of class $C_{i}$ for all $i=1, \ldots, n$, with $\sum_{1 \leq i \leq n} p_{i}=1$.

Recalling that the equivalence classes with respect to $\sim_{\mathrm{PB}, \mathrm{gbg},=}$ are unions of equivalence classes with respect to $\sim \mathrm{PB}$,dis due to Thm. 5(1), we focus on:

$$
\mathscr{G}=\left\{C^{\prime} \in\left(S \|_{A} S\right) / \sim_{\mathrm{PB}, \mathrm{gbg},=} \mid \exists C \in \mathscr{C} .\left(C \|_{A} C\right) \subseteq C^{\prime}\right\}
$$

and prove that the transition $s_{1} \|_{A} s \stackrel{a}{\longrightarrow} \mathscr{D}_{1}^{\prime}$, deriving from the synchronization of the transition $s_{1} \stackrel{a}{\longrightarrow} \mathscr{D}_{1}$ with the transition $s \stackrel{a}{\longrightarrow} \mathscr{D}$, is such that for each transition $s_{2} \|_{A} s \stackrel{a}{\longrightarrow} \mathscr{D}_{2}^{\prime}$, deriving from the synchronization of one of the $m$ transitions $s_{2} \stackrel{a}{\longrightarrow} \mathscr{D}_{2}$ with the transition $s \stackrel{a}{\longrightarrow} \mathscr{D}$, it holds that $\mathscr{D}_{1}^{\prime}(\bigcup \mathscr{G}) \neq \mathscr{D}_{2}^{\prime}(\bigcup \mathscr{G})$. There are two cases:

- If the set of actions labeling the outgoing transitions of $s_{C_{i}}$ is disjoint from the set of actions labeling the outgoing transitions of $s_{C_{j}}$ for all $i \neq j$, then $s_{C_{i}} \|_{A} s_{C_{j}}$ is a terminal state not belonging to $\bigcup \mathscr{G}$ and for each of the $m$ transitions $s_{2} \|_{A} s \stackrel{a}{\longrightarrow} \mathscr{D}_{2}^{\prime}$ it holds that $\mathscr{D}_{1}^{\prime}(\bigcup \mathscr{G}) \neq \mathscr{D}_{2}^{\prime}(\bigcup \mathscr{G})$, i.e., $\mathscr{D}_{1}^{\prime}(\bigcup \mathscr{G})-\mathscr{D}_{2}^{\prime}(\bigcup \mathscr{G}) \neq 0$, iff:

$$
\sum_{i=1}^{n} p_{i} \cdot \mathscr{D}_{1}\left(C_{i}\right)-\sum_{i=1}^{n} p_{i} \cdot \mathscr{D}_{2}\left(C_{i}\right)=\sum_{i=1}^{n} p_{i} \cdot\left(\mathscr{D}_{1}\left(C_{i}\right)-\mathscr{D}_{2}\left(C_{i}\right)\right) \neq 0
$$

which yields a system of $m$ inequalities with respect to the $n$ unknowns $p_{1}, p_{2}, \ldots, p_{n}$. 
Each of the associated equations:

$$
\sum_{i=1}^{n} p_{i} \cdot\left(\mathscr{D}_{1}\left(C_{i}\right)-\mathscr{D}_{2}\left(C_{i}\right)\right)=0
$$

describes a hyperplane that separates the space $\mathbb{R}^{n}$ into two half spaces (depending on whether we impose $>0$ or $<0$ ) because at least one of the coefficients $\mathscr{D}_{1}\left(C_{i}\right)-\mathscr{D}_{2}\left(C_{i}\right)$ is different from 0 (see the initial part of the proof). Since the number of inequalities/hyperplanes is finite, there exists a nonempty subspace of $\mathbb{R}^{n}$ identified by the hyperplanes themselves, whose points satisfy all the $m$ inequalities. If we take one point of the subspace that does not belong to any of the hyperplanes and whose coordinates are all not negative, its normalization to 1 (obtained by dividing each coordinate of the selected point by the sum of its coordinates) is again a point that satisfies all the $m$ inequalities; notice that the normalized point belongs to the hyperplane $\sum_{1 \leq i \leq n} p_{i}=1$ and that such a hyperplane does not contain the origin of $\mathbb{R}^{n}$, while each of the $m$ considered hyperplanes contains the origin, hence the normalized point does not belong to any of those hyperplanes. This proves the existence of a probability distribution $p_{1}, p_{2}, \ldots, p_{n}$ satisfying all the $m$ inequalities.

- If the set of actions labeling the outgoing transitions of $s_{C_{i}}$ is not disjoint from the set of actions labeling the outgoing transitions of $s_{C_{j}}$ for some $i \neq j$, then it is sufficient to decorate each "shared" action labeling a transition from one of those states with the name of the equivalence class with respect to $\sim_{\mathrm{PB} \text {, dis }}$ to which the source state of the transition belongs. Observing that two states are related by $\sim_{\mathrm{PB}, \mathrm{dis}}$ iff so are their "decorated" versions, we can then proceed as in the previous case.

With the same proof strategy, we can show that $\sim_{\mathrm{PB}, \mathrm{dis}}^{\mathrm{ct}}$ is the coarsest congruence contained in $\sim_{\mathrm{PB}, \mathrm{gbg},=}^{\mathrm{ct}}$, where the latter coincides with $\sim_{\mathrm{PB}, \mathrm{gbg}, \leq}$ by virtue of Thms. 6 and 5 as $\mathscr{L}$ is image finite.

\section{Conclusion}

We have addressed the problem of defining behavioral relations for nondeterministic and probabilistic processes that are characterized by modal logics as close as possible to PML, the natural probabilistic extension of the by now standard modal logic HML for fully nondeterministic processes. We have focussed on two new probabilistic bisimilarities, denoted by $\sim_{\mathrm{PB}, \mathrm{gbg},=}$ and $\sim_{\mathrm{PB}, \mathrm{gbg}, \leq}$, following a so called group-by-group approach that considers groups of equivalence classes rather than single classes. Interestingly enough, one of these equivalences $\left(\sim_{\mathrm{PB}, \mathrm{gbg},=}\right)$ has independently emerged in a framework recently developed to provide a uniform model for nondeterministic, probabilistic, and stochastic processes [5].

For the proposed equivalences, we have studied the relationships with an existential and a universal interpretation of three variants of PML, in which the diamond is respectively decorated with a probability interval, a probability lower bound, or a probability upper bound. We have shown that five out of the six logical equivalences do coincide with $\sim_{\mathrm{PB}, \mathrm{gbg}, \leq}$. The only exception is the logic based on existential interpretation and probability intervals, which corresponds to $\sim_{\mathrm{PB}, \mathrm{gbg},=}$. This indicates that, in PML, adopting a universal interpretation rather than an existential one does not matter, for comparison purposes, as long as diamond operators are decorated with probability bounds.

In the group-by-group approach, the three initially introduced probabilistic bisimilarities, whose definitions differ for the requirements on the comparison between sets of probabilities $(=, \leq, \geq)$, have been equipped with alternative characterizations obtained by simply 
comparing extremal probabilities (resp. both $\sqcup$ and $\sqcap$, only $\sqcup$, only $\sqcap$ ). We have also considered six variants relying on combined transitions. Quite surprisingly, all such variants, except the one based on $=$, coincide with the relations relying on ordinary transitions. This result is particularly interesting because it shows that, in our approach, resolving nondeterminism with deterministic schedulers or with randomized ones leads to the same identifications, except when checking probabilities for equality in the bisimulation game.

Our modal logic characterizations and backward compatibility results for $\sim_{\mathrm{PB}, \mathrm{gbg}, \leq}$, together with the modal logic characterizations of probabilistic bisimilarity over reactive probabilistic processes $[33,34]$ and of class-distribution probabilistic bisimilarity over alternating processes [35], show that PML constitutes a uniform framework for reasoning on different classes of processes including probability and various degrees of nondeterminism.

Our work has a number of points in common with [42], where new probabilistic bisimilarities over nondeterministic and probabilistic Kripke structures have been defined that are in full agreement with PCTL and PCTL*. Indeed, both [42] and our work witness that, in a nondeterministic and probabilistic setting, the equivalences induced by PCTL/PCTL*/PML are not compositional - with the probabilistic bisimilarities defined in [39] being the coarsest congruences contained in all of them - and that, to characterize them, it is necessary to:

- Anticipate the quantification over the sets of equivalent states to be reached in the bisimulation game, as done in [43] in the setting of approximate probabilistic relations. Placing this quantification after the comparison of probability values, like in [39], results in a much finer probabilistic bisimilarity that needs a modal logic much more expressive than PML as shown in $[35,27,25]$.

- Consider groups of classes of equivalent states rather than only individual classes, as done in $[18,9,13]$ in the setting of bisimilarity for continuous-state probabilistic processes. Otherwise, due to the anticipation of the previously mentioned quantification, a much coarser probabilistic bisimilarity would be obtained (see Sect. 5.1).

- Focus only on the extremal probabilities of reaching certain sets of states or, equivalently, check the probabilities of reaching those sets of states for $\leq$ or $\geq$ rather than $=$. In this way, the presence of nondeterminism within processes and the probabilistic bounds in logical formulae fit well together.

Our results and those of [42] also show that, in the case of nondeterministic and probabilistic processes, it is not possible to define a single probabilistic bisimilarity that is characterized by both PML - interpreted as in this paper - and PCTL* - interpreted as in [7]. Thus, for nondeterministic and probabilistic processes the situation is quite different from the case of fully nondeterministic processes, where probabilistic bisimilarity is characterized by both HML [26] and CTL* [8], and from the case of reactive probabilistic processes, where probabilistic bisimilarity is characterized by both PML [33,34] and PCTL* [1].

As for other related work, in the more general framework of category theory many papers have investigated various types of probabilistic processes as coalgebras of suitable functors together with the corresponding notions of bisimilarity (see, e.g., [20,45,3]). Moreover, modal logics have been given a clean interpretation in coalgebras $[31,32,10]$, even on measurable spaces [22]. Although the generality of the categorical approach does clarify many connections, it does not necessarily lead to solutions better than a "hand-made", specificallytailored bisimilarity or modal logic. Therefore, in this paper we have preferred a more concrete and direct approach, which is accessible to a wider audience.

As future work, we plan to examine further properties of group-by-group probabilistic bisimilarities. Results along this direction would also be useful for a better understanding of the probabilistic bisimilarities of [42]. Likewise, it would be interesting to consider the new 
probabilistic bisimilarities in a continuous-state setting, to see whether measure-theoretic machinery would allow us to dispense with the assumptions of image finiteness and minimal probability on which our characterization results rely in the discrete-state setting. Another obvious direction of research would be to define the weak variants of the group-by-group probabilistic bisimilarities and find the corresponding modal logics. Finally, we would like to study expressiveness of the variants of PML introduced in this paper in terms of the systems properties they can model.

Acknowledgements We are grateful to the anonymous referees for their stimulating comments. We would like to thank Devis Abriani for his useful suggestions on the proof of the coarsest congruence results. This work has been partially supported by the FP7-IST-FET Project ASCENS, grant no. 257414, by the EU Project QUANTICOL, grant no. 600708, and by the MIUR-PRIN Project CINA.

\section{A Multistep Variants Inspired by $\sim_{B}$}

We start by formalizing the notion of computation in the NPLTS setting as a sequence of state-to-state steps each derived from a state-to-distribution transition.

Definition 11 Let $\mathscr{L}=(S, A, \longrightarrow)$ be an NPLTS. A sequence $c \equiv s_{0} \stackrel{a_{1}}{\rightarrow} s_{1} \stackrel{a_{2}}{\longrightarrow} s_{2} \ldots s_{n-1} \stackrel{a_{n}}{\rightarrow} s_{n}$ is a computation of $\mathscr{L}$ of length $n$ going from $s_{0}$ to $s_{n}$ iff for all $i=1, \ldots, n$ there exists a transition $s_{i-1} \stackrel{a_{i}}{\longrightarrow} \mathscr{D}_{i}$ such that $s_{i} \in \operatorname{supp}\left(\mathscr{D}_{i}\right)$, with $\mathscr{D}_{i}\left(s_{i}\right)$ being the execution probability of step $s_{i-1} \stackrel{a_{i}}{\longrightarrow} s_{i}$ of $c$ conditioned on the selection of transition $s_{i-1} \stackrel{a_{i}}{\longrightarrow} \mathscr{D}_{i}$ of $\mathscr{L}$ at state $s_{i-1}$; in this case, we write $s_{0} \stackrel{\alpha}{\Longrightarrow} \mathscr{D}_{n}$ where $\alpha=a_{1} a_{2} \ldots a_{n}$, with $s_{0} \stackrel{\varepsilon}{\Longrightarrow} \delta_{s_{0}}$ when $\alpha=\varepsilon$. We call combined computation a computation in which every step arises from a combined transition, denoted by $\Longrightarrow_{\mathrm{c}}$.

We now introduce the multistep variant of $\sim_{\mathrm{B}}$ and prove that it coincides with $\sim_{\mathrm{B}}$ itself.

Definition 12 Let $(S, A, \longrightarrow)$ be an NPLTS in which the target of each transition is a Dirac distribution. A relation $\mathscr{B}$ over $S$ is a multistep bisimulation iff, whenever $\left(s_{1}, s_{2}\right) \in \mathscr{B}$, then for all traces $\alpha \in A^{*}$ :

- For each $s_{1} \stackrel{\alpha}{\Longrightarrow} \delta_{s_{1}^{\prime}}$ there exists $s_{2} \stackrel{\alpha}{\Longrightarrow} \delta_{s_{2}^{\prime}}$ such that $\left(s_{1}^{\prime}, s_{2}^{\prime}\right) \in \mathscr{B}$.

- For each $s_{2} \stackrel{\alpha}{\Longrightarrow} \delta_{s_{2}^{\prime}}$ there exists $s_{1} \stackrel{\alpha}{\Longrightarrow} \delta_{s_{1}^{\prime}}$ such that $\left(s_{1}^{\prime}, s_{2}^{\prime}\right) \in \mathscr{B}$.

We denote by $\sim_{\mathrm{B}, \mathrm{m}}$ the largest multistep bisimulation.

Theorem 8 Let $(S, A, \longrightarrow)$ be an NPLTS in which the target of each transition is a Dirac distribution. Let $s_{1}, s_{2} \in S$. Then:

$$
s_{1} \sim_{\mathrm{B}, \mathrm{m}} s_{2} \Longleftrightarrow s_{1} \sim_{\mathrm{B}} s_{2}
$$

Proof Suppose that $s_{1} \sim_{\mathrm{B}, \mathrm{m}} s_{2}$. This means that there exists a multistep bisimulation $\mathscr{B}$ over $S$ such that $\left(s_{1}, s_{2}\right) \in \mathscr{B}$. As a consequence, it holds in particular that, whenever $\left(s_{1}^{\prime}, s_{2}^{\prime}\right) \in \mathscr{B}$, then for all a $\in A$ :

- For each $s_{1}^{\prime} \stackrel{a}{\Longrightarrow} \delta_{s_{1}^{\prime \prime}}$ there exists $s_{2}^{\prime} \stackrel{a}{\Longrightarrow} \delta_{s_{2}^{\prime \prime}}$ such that $\left(s_{1}^{\prime \prime}, s_{2}^{\prime \prime}\right) \in \mathscr{B}$.

- For each $s_{2}^{\prime} \stackrel{a}{\Longrightarrow} \delta_{s_{2}^{\prime \prime}}$ there exists $s_{1}^{\prime} \stackrel{a}{\Longrightarrow} \delta_{s_{1}^{\prime \prime}}$ such that $\left(s_{1}^{\prime \prime}, s_{2}^{\prime \prime}\right) \in \mathscr{B}$.

Since $\stackrel{a}{\Longrightarrow}$ coincides with $\stackrel{a}{\longrightarrow}$, we have that $\mathscr{B}$ is also a bisimulation and hence $s_{1} \sim_{\mathrm{B}} s_{2}$

Suppose now that $s_{1} \sim_{\mathrm{B}} s_{2}$. This means that there exists a bisimulation $\mathscr{B}$ over $S$ such that $\left(s_{1}, s_{2}\right) \in \mathscr{B}$. We prove that $\mathscr{B}$ is also a multistep bisimulation, so that $s_{1} \sim_{\mathrm{B}, \mathrm{m}} s_{2}$ will follow. Given $s_{1}^{\prime}, s_{2}^{\prime} \in S$ such that $\left(s_{1}^{\prime}, s_{2}^{\prime}\right) \in \mathscr{B}$ and $\alpha \in A^{*}$, we proceed by induction on $|\alpha|$ :

- If $|\alpha|=0$, then $s_{1}^{\prime} \stackrel{\alpha}{\Longrightarrow} \delta_{s_{1}^{\prime}}$ and $s_{2}^{\prime} \stackrel{\alpha}{\Longrightarrow} \delta_{s_{2}^{\prime}}$ are the only possible computations from $s_{1}^{\prime}$ and $s_{2}^{\prime}$ labeled with $\alpha$, hence the result trivially holds. 
- Let $|\alpha|=n \in \mathbb{N}_{>0}$ and suppose that the result holds for all traces of length $n-1$. Assume $\alpha=a \alpha^{\prime}$. Since $\left(s_{1}^{\prime}, s_{2}^{\prime}\right) \in \mathscr{B}$ and $\mathscr{B}$ is a bisimulation, it holds that for each $s_{1}^{\prime} \stackrel{a}{\longrightarrow} \delta_{s_{1}^{\prime \prime \prime}}$ there exists $s_{2}^{\prime} \stackrel{a}{\longrightarrow} \delta_{s_{2}^{\prime \prime \prime}}$ (and vice versa) such that $\left(s_{1}^{\prime \prime \prime}, s_{2}^{\prime \prime \prime}\right) \in \mathscr{B}$.

Suppose that $s_{1}^{\prime} \stackrel{\alpha}{\longrightarrow} \delta_{s_{1}^{\prime \prime}}$ with $s_{1}^{\prime} \stackrel{a}{\longrightarrow} \delta_{s_{1}^{\prime \prime \prime}}$ and $s_{1}^{\prime \prime \prime} \stackrel{\alpha^{\prime}}{\longrightarrow} \delta_{s_{1}^{\prime \prime}}$. Then $s_{2}^{\prime} \stackrel{a}{\longrightarrow} \delta_{s_{2}^{\prime \prime \prime}}$ with $\left(s_{1}^{\prime \prime \prime}, s_{2}^{\prime \prime \prime}\right) \in \mathscr{B}$ and by the induction hypothesis we have that $s_{2}^{\prime \prime \prime} \stackrel{\alpha^{\prime}}{\Longrightarrow} \delta_{s_{2}^{\prime \prime}}$ with $\left(s_{1}^{\prime \prime}, s_{2}^{\prime \prime}\right) \in \mathscr{B}$. As a consequence, $s_{2}^{\prime} \stackrel{\alpha}{\Longrightarrow} \delta_{s_{2}^{\prime \prime}}$ with $\left(s_{1}^{\prime \prime}, s_{2}^{\prime \prime}\right) \in \mathscr{B}$. With a similar argument, we derive that $s_{2}^{\prime} \stackrel{\alpha}{\Longrightarrow} \delta_{s_{2}^{\prime \prime}}$ implies $s_{1}^{\prime} \stackrel{\alpha}{\Longrightarrow} \delta_{s_{1}^{\prime \prime}}$ with $\left(s_{1}^{\prime \prime}, s_{2}^{\prime \prime}\right) \in \mathscr{B}$.

We now provide the $\sim_{\mathrm{B}, \mathrm{m}}$-inspired definition of each of the probabilistic bisimilarities considered in this paper and prove that it coincides with the original one-step equivalence. The ct-variants of the $\sim_{\mathrm{B}, \mathrm{m}^{-}}$ inspired probabilistic bisimilarities can be defined similarly and satisfy an analogous coincidence property with respect to the original one-step ct-equivalences.

Definition 13 Let $(S, A, \longrightarrow)$ be an NPLTS. An equivalence relation $\mathscr{B}$ over $S$ is a multistep class-distribution probabilistic bisimulation iff, whenever $\left(s_{1}, s_{2}\right) \in \mathscr{B}$, then for all traces $\alpha \in A^{*}$ it holds that for each $s_{1} \stackrel{\alpha}{\Longrightarrow} \mathscr{D}_{1}$ there exists $s_{2} \stackrel{\alpha}{\Longrightarrow} \mathscr{D}_{2}$ such that, for all equivalence classes $C \in S / \mathscr{B}, \mathscr{D}_{1}(C)=\mathscr{D}_{2}(C)$. We denote by $\sim_{\mathrm{PB}, \mathrm{dis}, \mathrm{m}}$ the largest multistep class-distribution probabilistic bisimulation.

Theorem 9 Let $(S, A, \longrightarrow)$ be an NPLTS and $s_{1}, s_{2} \in S$. Then:

$$
s_{1} \sim_{\mathrm{PB}, \mathrm{dis}, \mathrm{m}} s_{2} \Longleftrightarrow s_{1} \sim_{\mathrm{PB}, \mathrm{dis}} s_{2}
$$

Proof Suppose that $s_{1} \sim \mathrm{PB}$ dis, $s_{2}$. This means that there exists a multistep class-distribution probabilistic bisimulation $\mathscr{B}$ over $S$ such that $\left(s_{1}, s_{2}\right) \in \mathscr{B}$. As a consequence, we have in particular that, whenever $\left(s_{1}^{\prime}, s_{2}^{\prime}\right) \in \mathscr{B}$, then for all $a \in A$ it holds that for each $s_{1}^{\prime} \stackrel{a}{\Longrightarrow} \mathscr{D}_{1}$ there exists $s_{2}^{\prime} \stackrel{a}{\Longrightarrow} \mathscr{D}_{2}$ such that, for all $C \in S / \mathscr{B}, \mathscr{D}_{1}(C)=\mathscr{D}_{2}(C)$. Since $\stackrel{a}{\Longrightarrow}$ coincides with $\stackrel{a}{\longrightarrow}$, we have that $\mathscr{B}$ is also a class-distribution probabilistic bisimulation and hence $s_{1} \sim \mathrm{PB}$, dis $s_{2}$.

Suppose now that $s_{1} \sim \mathrm{PB}$,dis $s_{2}$. This means that there exists a class-distribution probabilistic bisimulation $\mathscr{B}$ over $S$ such that $\left(s_{1}, s_{2}\right) \in \mathscr{B}$. We prove that $\mathscr{B}$ is also a multistep class-distribution probabilistic bisimulation, so that $s_{1} \sim_{\mathrm{PB}, \mathrm{dis}, \mathrm{m}} s_{2}$ will follow. Given $s_{1}^{\prime}, s_{2}^{\prime} \in S$ such that $\left(s_{1}^{\prime}, s_{2}^{\prime}\right) \in \mathscr{B}$ and $\alpha \in A^{*}$, we proceed by induction on $|\alpha|$ :

- If $|\alpha|=0$, then $s_{1}^{\prime} \stackrel{\alpha}{\Longrightarrow} \delta_{s_{1}^{\prime}}$ and $s_{2}^{\prime} \stackrel{\alpha}{\Longrightarrow} \delta_{s_{2}^{\prime}}$ are the only possible computations from $s_{1}^{\prime}$ and $s_{2}^{\prime}$ labeled with $\alpha$ and for all $C \in S / \mathscr{B}$ it holds that:

$$
\delta_{s_{1}^{\prime}}(C)=\delta_{s_{2}^{\prime}}(C)= \begin{cases}1 & \text { if }\left\{s_{1}^{\prime}, s_{2}^{\prime}\right\} \subseteq C \\ 0 & \text { if }\left\{s_{1}^{\prime}, s_{2}^{\prime}\right\} \cap C=\emptyset\end{cases}
$$

because $\left(s_{1}^{\prime}, s_{2}^{\prime}\right) \in \mathscr{B}$ and $C$ is an equivalence class with respect to $\mathscr{B}$.

- Let $|\alpha|=n \in \mathbb{N}_{>0}$ and suppose that the result holds for all traces of length $n-1$. Assume $\alpha=a \alpha^{\prime}$. Since $\left(s_{1}^{\prime}, s_{2}^{\prime}\right) \in \mathscr{B}$ and $\mathscr{B}$ is a class-distribution probabilistic bisimulation, it holds that for each $s_{1}^{\prime} \stackrel{a}{\longrightarrow} \mathscr{D}_{1}^{\prime}$ there exists $s_{2}^{\prime} \stackrel{a}{\longrightarrow} \mathscr{D}_{2}^{\prime}$ such that, for all $C \in S / \mathscr{B}, \mathscr{D}_{1}^{\prime}(C)=\mathscr{D}_{2}^{\prime}(C)$.

Suppose that $s_{1}^{\prime} \stackrel{\alpha}{\Longrightarrow} \mathscr{D}_{1}$ with $s_{1}^{\prime} \stackrel{a}{\longrightarrow} \mathscr{D}_{1}^{\prime}, s_{1}^{\prime \prime} \stackrel{\alpha^{\prime}}{\longrightarrow} \mathscr{D}_{1}$, and $\mathscr{D}_{1}^{\prime}\left(s_{1}^{\prime \prime}\right)>0$. Then there exists $s_{2}^{\prime} \stackrel{a}{\longrightarrow} \mathscr{D}_{2}^{\prime}$ such that, for all $C \in S / \mathscr{B}, \mathscr{D}_{1}^{\prime}(C)=\mathscr{D}_{2}^{\prime}(C)$. If we take $s_{2}^{\prime \prime}$ such that $\left(s_{1}^{\prime \prime}, s_{2}^{\prime \prime}\right) \in \mathscr{B}$ and $\mathscr{D}_{2}^{\prime}\left(s_{2}^{\prime \prime}\right)>0$, by the induction hypothesis there exists $s_{2}^{\prime \prime} \stackrel{\alpha^{\prime}}{\Longrightarrow} \mathscr{D}_{2}$ such that, for all $C \in S / \mathscr{B}, \mathscr{D}_{1}(C)=\mathscr{D}_{2}(C)$. As a consequence, there exists $s_{2}^{\prime} \stackrel{\alpha}{\Longrightarrow} \mathscr{D}_{2}$ such that, for all $C \in S / \mathscr{B}, \mathscr{D}_{1}(C)=\mathscr{D}_{2}(C)$.

Definition 14 Let $(S, A, \longrightarrow)$ be an NPLTS and $\bowtie \in\{=, \leq, \geq\}$. An equivalence relation $\mathscr{B}$ over $S$ is a multistep $\bowtie$-group-by-group probabilistic bisimulation iff, whenever $\left(s_{1}, s_{2}\right) \in \mathscr{B}$, then for all traces $\alpha \in A^{*}$ and for all groups of equivalence classes $\mathscr{G} \in 2^{S / \mathscr{B}}$ it holds that for each $s_{1} \stackrel{\alpha}{\Longrightarrow} \mathscr{D}_{1}$ there exists $s_{2} \stackrel{\alpha}{\Longrightarrow} \mathscr{D}_{2}$ such that $\mathscr{D}_{1}(\bigcup \mathscr{G}) \bowtie \mathscr{D}_{2}(\bigcup \mathscr{G})$. We denote by $\sim_{\mathrm{PB}, \mathrm{gbg}, \bowtie, \mathrm{m}}$ the largest multistep $\bowtie$-group-by-group probabilistic bisimulation.

Theorem 10 Let $(S, A, \longrightarrow)$ be an NPLTS, $s_{1}, s_{2} \in S$, and $\bowtie \in\{=, \leq, \geq\}$. Then:

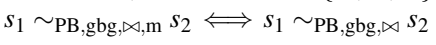

Proof Suppose that $s_{1} \sim \mathrm{PB}, \mathrm{gbg}, \mathrm{m}_{\mathrm{m}} s_{2}$. This means that there exists a multistep $\bowtie$-group-by-group probabilistic bisimulation $\mathscr{B}$ over $S$ such that $\left(s_{1}, s_{2}\right) \in \mathscr{B}$. As a consequence, we have in particular that, whenever $\left(s_{1}^{\prime}, s_{2}^{\prime}\right) \in \mathscr{B}$, then for all $a \in A$ and $\mathscr{G} \in 2^{S / \mathscr{B}}$ it holds that for each $s_{1}^{\prime} \stackrel{a}{\Longrightarrow} \mathscr{D}_{1}$ there exists $s_{2}^{\prime} \stackrel{a}{\Longrightarrow} \mathscr{D}_{2}$ such that 
$\mathscr{D}_{1}(\bigcup \mathscr{G}) \bowtie \mathscr{D}_{2}(\bigcup \mathscr{G})$. Since $\stackrel{a}{\Longrightarrow}$ coincides with $\stackrel{a}{\longrightarrow}$, we have that $\mathscr{B}$ is also a $\bowtie$-group-by-group probabilistic bisimulation and hence $s_{1} \sim \mathrm{PB}, \mathrm{gbg}, \bowtie s_{2}$.

Suppose now that $s_{1} \sim \mathrm{PB}, \mathrm{gbg}, \bowtie s_{2}$. This means that there exists a $\bowtie$-group-by-group probabilistic bisimulation $\mathscr{B}$ over $S$ such that $\left(s_{1}, s_{2}\right) \in \mathscr{B}$. We prove that $\mathscr{B}$ is also a multistep $\bowtie$-group-by-group probabilistic bisimulation, so that $s_{1} \sim_{\mathrm{PB}, \mathrm{gbg}, \bowtie, \mathrm{m}} s_{2}$ will follow. Given $s_{1}^{\prime}, s_{2}^{\prime} \in S$ such that $\left(s_{1}^{\prime}, s_{2}^{\prime}\right) \in \mathscr{B}, \alpha \in A^{*}$, and $\mathscr{G} \in 2^{S / \mathscr{B}}$, we proceed by induction on $|\alpha|$ :

- If $|\alpha|=0$, then $s_{1}^{\prime} \stackrel{\alpha}{\Longrightarrow} \delta_{s_{1}^{\prime}}$ and $s_{2}^{\prime} \stackrel{\alpha}{\Longrightarrow} \delta_{s_{2}^{\prime}}$ are the only possible computations from $s_{1}^{\prime}$ and $s_{2}^{\prime}$ labeled with $\alpha$ and it holds that:

$$
\delta_{s_{1}^{\prime}}(\cup \mathscr{G})=\delta_{s_{2}^{\prime}}(\cup \mathscr{G})= \begin{cases}1 & \text { if }\left\{s_{1}^{\prime}, s_{2}^{\prime}\right\} \subseteq C \text { for some } C \in \mathscr{G} \\ 0 & \text { if }\left\{s_{1}^{\prime}, s_{2}^{\prime}\right\} \cap C=\emptyset \text { for all } C \in \mathscr{G}\end{cases}
$$

because $\left(s_{1}^{\prime}, s_{2}^{\prime}\right) \in \mathscr{B}$ and $\mathscr{G}$ is a group of equivalence classes with respect to $\mathscr{B}$.

- Let $|\alpha|=n \in \mathbb{N}_{>0}$ and suppose that the result holds for all traces of length $n-1$. Assume $\alpha=a \alpha^{\prime}$. Since $\left(s_{1}^{\prime}, s_{2}^{\prime}\right) \in \mathscr{B}$ and $\mathscr{B}$ is a $\bowtie$-group-by-group probabilistic bisimulation, for all $\mathscr{G}^{\prime} \in 2^{S / \mathscr{B}}$ it holds that for each $s_{1}^{\prime} \stackrel{a}{\longrightarrow} \mathscr{D}_{1}^{\prime}$ there exists $s_{2}^{\prime} \stackrel{a}{\longrightarrow} \mathscr{D}_{2}^{\prime}$ such that $\mathscr{D}_{1}^{\prime}\left(\bigcup \mathscr{G}^{\prime}\right) \bowtie \mathscr{D}_{2}^{\prime}\left(\bigcup^{\prime} \mathscr{G}^{\prime}\right)$.

Suppose that $s_{1}^{\prime} \stackrel{\alpha}{\Longrightarrow} \mathscr{D}_{1}$ with $s_{1}^{\prime} \stackrel{a}{\longrightarrow} \mathscr{D}_{1}^{\prime}, s_{1}^{\prime \prime} \stackrel{\alpha^{\prime}}{\Longrightarrow} \mathscr{D}_{1}$, and $\mathscr{D}_{1}^{\prime}\left(s_{1}^{\prime \prime}\right)>0$. Let $\mathscr{G}^{\prime}=\left\{C^{\prime}\right\}$ with $C^{\prime}$ being the equivalence class containing $s_{1}^{\prime \prime}$. Then there exists $s_{2}^{\prime} \stackrel{a}{\longrightarrow} \mathscr{D}_{2}^{\prime}$ such that $\mathscr{D}_{1}^{\prime}\left(\cup \mathscr{G}^{\prime}\right) \bowtie \mathscr{D}_{2}^{\prime}\left(\bigcup \mathscr{G}^{\prime}\right)$. If we take $s_{2}^{\prime \prime}$ such that $\left(s_{1}^{\prime \prime}, s_{2}^{\prime \prime}\right) \in \mathscr{B}$ and $\mathscr{D}_{2}^{\prime}\left(s_{2}^{\prime \prime}\right)>0-$ it obviously exists in the case that $\bowtie \in\{=, \leq\}$ because $\mathscr{D}_{1}^{\prime}\left(s_{1}^{\prime \prime}\right)>0$, and it also exists in the case that $\bowtie$ is $\geq$ because, if $s_{2}^{\prime}$ had no a-transition reaching $\mathscr{G}^{\prime}$ with probability greater than 0 , then all a-transitions of $s_{2}^{\prime}$ would reach $\mathscr{G}^{\prime \prime}=2^{S / \mathscr{B}} \backslash \mathscr{G}^{\prime}$ with probability 1 and hence for the transition $s_{1}^{\prime} \stackrel{a}{\longrightarrow} \mathscr{D}_{1}^{\prime}$ we would have $\mathscr{D}_{1}^{\prime}\left(\bigcup \mathscr{G}^{\prime \prime}\right)=1-\mathscr{D}_{1}^{\prime}\left(\bigcup \mathscr{G}^{\prime}\right)<1=\mathscr{D}_{2}^{\prime}\left(\bigcup \mathscr{G}^{\prime \prime}\right)$ for all transitions $s_{2}^{\prime} \stackrel{a}{\longrightarrow} \mathscr{D}_{2}^{\prime}$, i.e., $\mathscr{B}$ would not be a $\geq$-group-by-group probabilistic bisimulation - by the induction hypothesis there exists $s_{2}^{\prime \prime} \stackrel{\alpha^{\prime}}{\Longrightarrow} \mathscr{D}_{2}$ such that $\mathscr{D}_{1}(\bigcup \mathscr{G}) \bowtie \mathscr{D}_{2}(\bigcup \mathscr{G})$. As a consequence, there exists $s_{2}^{\prime} \stackrel{\alpha}{\Longrightarrow} \mathscr{D}_{2}$ such that $\mathscr{D}_{1}(\bigcup \mathscr{G}) \bowtie \mathscr{D}_{2}(\bigcup \mathscr{G})$.

Definition 15 Let $(S, A, \longrightarrow)$ be an NPLTS. An equivalence relation $\mathscr{B}$ over $S$ is a multistep $\sqcup \sqcap$-group-bygroup probabilistic bisimulation iff, whenever $\left(s_{1}, s_{2}\right) \in \mathscr{B}$, then for all traces $\alpha \in A^{*}$ and for all groups of equivalence classes $\mathscr{G} \in 2^{S / \mathscr{B}}$ it holds that $s_{1} \stackrel{\alpha}{\Longrightarrow}$ iff $s_{2} \stackrel{\alpha}{\Longrightarrow}$ and:

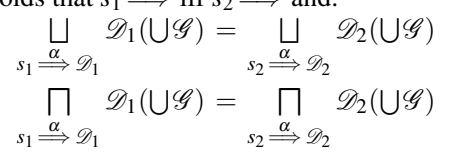

We denote by $\sim_{\mathrm{PB}, \mathrm{gbg}, \sqcup \sqcap, \mathrm{m}}$ the largest multistep $\sqcup \sqcap$-group-by-group probabilistic bisimulation.

Theorem 11 Let $(S, A, \longrightarrow)$ be an NPLTS and $s_{1}, s_{2} \in S$. Then:

$$
s_{1} \sim_{\mathrm{PB}, \mathrm{gbg}, \sqcup \sqcap, \mathrm{m}} s_{2} \Longleftrightarrow s_{1} \sim_{\mathrm{PB}, \mathrm{gbg}, \sqcup \sqcap} s_{2}
$$

Proof Suppose that $s_{1} \sim \mathrm{PB}, \mathrm{gbg}, \sqcup \sqcap, \mathrm{m} s_{2}$. This means that there exists a multistep $\sqcup \sqcap$-group-by-group probabilistic bisimulation $\mathscr{B}$ over $S$ such that $\left(s_{1}, s_{2}\right) \in \mathscr{B}$. As a consequence, we have in particular that, whenever $\left(s_{1}^{\prime}, s_{2}^{\prime}\right) \in \mathscr{B}$, then for all $a \in A$ and $\mathscr{G} \in 2^{S / \mathscr{B}}$ it holds that $s_{1}^{\prime} \stackrel{a}{\Longrightarrow}$ iff $s_{2}^{\prime} \stackrel{a}{\Longrightarrow}$ and:

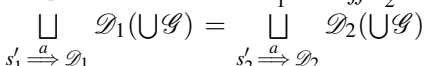

$$
\begin{aligned}
& \prod_{s_{1}^{\prime} \stackrel{a}{\Longrightarrow} \mathscr{D}_{1}} \mathscr{D}_{1}(\bigcup \mathscr{G})=\prod_{s_{2}^{\prime} \stackrel{a}{\Longrightarrow} \mathscr{D}_{2}} \mathscr{D}_{2}(\bigcup \mathscr{G})
\end{aligned}
$$

Since $\stackrel{a}{\Longrightarrow}$ coincides with $\stackrel{a}{\longrightarrow}$, we have that $\mathscr{B}$ is also $a \sqcup \sqcap$-group-by-group probabilistic bisimulation and hence $s_{1} \sim \mathrm{PB}, \mathrm{gbg}, \sqcup \sqcap s_{2}$.

Suppose now that $s_{1} \sim \mathrm{PB}, \mathrm{gbg}, \sqcup \sqcap s_{2}$. This means that there exists a $\square$-group-by-group probabilistic bisimulation $\mathscr{B}$ over $S$ such that $\left(s_{1}, s_{2}\right) \in \mathscr{B}$. We prove that $\mathscr{B}$ is also a multistep $\sqcup \Pi$-group-by-group probabilistic bisimulation, so that $s_{1} \sim \mathrm{PB}, \mathrm{gbg}, \sqcup \sqcap, \mathrm{m} s_{2}$ will follow. Given $s_{1}^{\prime}, s_{2}^{\prime} \in S$ such that $\left(s_{1}^{\prime}, s_{2}^{\prime}\right) \in \mathscr{B}, \alpha \in A^{*}$, and $\mathscr{G} \in 2^{S / \mathscr{B}}$, we proceed by induction on $|\alpha|$ :

- If $|\alpha|=0$, then $s_{1}^{\prime} \stackrel{\alpha}{\Longrightarrow} \delta_{s_{1}^{\prime}}$ and $s_{2}^{\prime} \stackrel{\alpha}{\Longrightarrow} \delta_{s_{2}^{\prime}}$ are the only possible computations from $s_{1}^{\prime}$ and $s_{2}^{\prime}$ labeled with $\alpha$ and it holds that:

$$
\delta_{s_{1}^{\prime}}(\cup \mathscr{G})=\delta_{s_{2}^{\prime}}(\bigcup \mathscr{G})= \begin{cases}1 & \text { if }\left\{s_{1}^{\prime}, s_{2}^{\prime}\right\} \subseteq C \text { for some } C \in \mathscr{G} \\ 0 & \text { if }\left\{s_{1}^{\prime}, s_{2}^{\prime}\right\} \cap C=\emptyset \text { for all } C \in \mathscr{G}\end{cases}
$$

because $\left(s_{1}^{\prime}, s_{2}^{\prime}\right) \in \mathscr{B}$ and $\mathscr{G}$ is a group of equivalence classes with respect to $\mathscr{B}$. Therefore: 


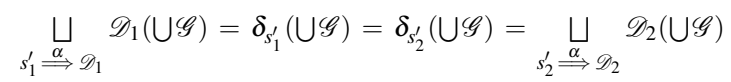

$$
\begin{aligned}
& \prod_{s_{1}^{\prime} \stackrel{\alpha}{\Longrightarrow} \mathscr{D}_{1}} \mathscr{D}_{1}(\bigcup \mathscr{G})=\delta_{s_{1}^{\prime}}(\bigcup \mathscr{G})=\delta_{s_{2}^{\prime}}(\bigcup \mathscr{G})=\prod_{s_{2}^{\prime} \stackrel{\alpha}{\Longrightarrow} \mathscr{D}_{2}} \mathscr{D}_{2}(\bigcup \mathscr{G})
\end{aligned}
$$

- Let $|\alpha|=n \in \mathbb{N}_{>0}$ and suppose that the result holds for all traces of length $n-1$. Assume $\alpha=a \alpha^{\prime}$. Since $\left(s_{1}^{\prime}, s_{2}^{\prime}\right) \in \mathscr{B}$ and $\mathscr{B}$ is a பП-group-by-group probabilistic bisimulation, for all $\mathscr{G}^{\prime} \in 2^{S / \mathscr{B}}$ it holds that $s_{1}^{\prime} \stackrel{a}{\longrightarrow}$ iff $s_{2}^{\prime} \stackrel{a}{\longrightarrow}$ and:

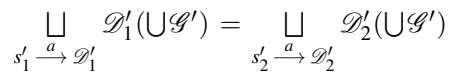

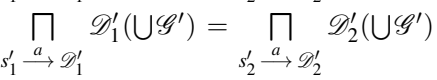

Suppose that $s_{1}^{\prime} \stackrel{\alpha}{\longrightarrow}$ with $s_{1}^{\prime} \stackrel{a}{\longrightarrow} \mathscr{D}_{1}^{\prime}, s_{1}^{\prime \prime} \stackrel{\alpha^{\prime}}{\longrightarrow}$, and $\mathscr{D}_{1}^{\prime}\left(s_{1}^{\prime \prime}\right)>0$. Let $\mathscr{G}^{\prime}=\left\{C^{\prime}\right\}$ with $C^{\prime}$ being the equivalence class containing $s_{1}^{\prime \prime}$. Then $s_{2}^{\prime} \stackrel{a}{\longrightarrow}$ with:

$$
\begin{aligned}
& \underset{s_{1}^{\prime} \underset{a}{\longrightarrow} \mathscr{D}_{1}^{\prime}}{\mathscr{D}_{1}^{\prime}\left(\bigcup^{\prime} \mathscr{G}^{\prime}\right)}=\underset{s_{2}^{\prime} \stackrel{a}{\longrightarrow} \mathscr{D}_{2}^{\prime}}{\mathscr{D}_{2}^{\prime}\left(\bigcup^{\prime} \mathscr{G}^{\prime}\right)} \\
& \prod_{s_{1}^{\prime} \stackrel{a}{\longrightarrow} \mathscr{D}_{1}^{\prime}} \mathscr{D}_{1}^{\prime}\left(\bigcup^{\prime}\right)=\prod_{s_{2}^{\prime} \underset{a}{\longrightarrow} \mathscr{D}_{2}^{\prime}} \mathscr{D}_{2}^{\prime}\left(\bigcup^{\prime} \mathscr{G}^{\prime}\right)
\end{aligned}
$$

If we take $s_{2}^{\prime \prime}$ and $\mathscr{D}_{2}^{\prime}$ such that $\left(s_{1}^{\prime \prime}, s_{2}^{\prime \prime}\right) \in \mathscr{B}, \mathscr{D}_{2}^{\prime}\left(s_{2}^{\prime \prime}\right)>0$, and $s_{2}^{\prime} \stackrel{a}{\longrightarrow} \mathscr{D}_{2}^{\prime}$, by the induction hypothesis we have that $s_{2}^{\prime \prime} \stackrel{\alpha^{\prime}}{\Longrightarrow}$ with:

As a consequence, $s_{2}^{\prime} \stackrel{\alpha}{\Longrightarrow}$ with:

$$
\begin{aligned}
& \sqcup \mathscr{D}_{1}(\bigcup \mathscr{G})=\sqcup \mathscr{D}_{2}(\bigcup \mathscr{G}) \\
& s_{1}^{\prime \prime} \stackrel{\alpha^{\prime}}{\Rightarrow} \mathscr{D}_{1} \quad s_{2}^{\prime \prime} \stackrel{\alpha^{\prime}}{\Rightarrow} \mathscr{D}_{2} \\
& \sqcap \mathscr{D}_{1}(\bigcup \mathscr{G})=\sqcap \mathscr{D}_{2}(\bigcup \mathscr{G}) \\
& s_{1}^{\prime \prime} \stackrel{\alpha^{\prime}}{\Rightarrow} \mathscr{D}_{1} \quad s_{2}^{\prime \prime} \stackrel{\alpha^{\prime}}{\Longrightarrow} \mathscr{D}_{2}
\end{aligned}
$$

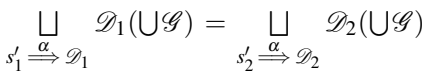

$$
\begin{aligned}
& \prod_{\alpha} \mathscr{D}_{1}(\bigcup \mathscr{G})=\prod_{\alpha} \mathscr{D}_{2}(\bigcup \mathscr{G})
\end{aligned}
$$

Definition 16 Let $(S, A, \longrightarrow)$ be an NPLTS and \# $\in\left\{\bigsqcup, \prod\right\}$. An equivalence relation $\mathscr{B}$ over $S$ is a multistep \#-group-by-group probabilistic bisimulation iff, whenever $\left(s_{1}, s_{2}\right) \in \mathscr{B}$, then for all traces $\alpha \in A^{*}$ and for all groups of equivalence classes $\mathscr{G} \in 2^{S / \mathscr{B}}$ it holds that $s_{1} \stackrel{\alpha}{\Longrightarrow}$ iff $s_{2} \stackrel{\alpha}{\Longrightarrow}$ and:

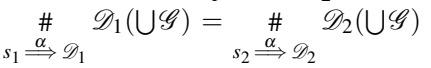

We denote by $\sim$ PB,gbg,\#,m the largest multistep \#-group-by-group probabilistic bisimulation.

Theorem 12 Let $(S, A, \longrightarrow)$ be an NPLTS, $s_{1}, s_{2} \in S$, and $\# \in\left\{\sqcup, \prod\right\}$. Then:

$$
s_{1} \sim \mathrm{PB}, \mathrm{gbg}, \#, \mathrm{~m} s_{2} \Longleftrightarrow s_{1} \sim \mathrm{PB}, \mathrm{gbg}, \# s_{2}
$$

Proof Similar to the proof of Thm. 11. With regard to the induction step of the proof that $s_{1} \sim_{\mathrm{PB}, \mathrm{gbg}, \#} s_{2}$ implies $s_{1} \sim_{\mathrm{PB}, \mathrm{gbg}, \#, \mathrm{~m}} s_{2}$, we observe that $s_{2}^{\prime \prime}$ and $\mathscr{D}_{2}^{\prime}$ such that $\left(s_{1}^{\prime \prime}, s_{2}^{\prime \prime}\right) \in \mathscr{B}, \mathscr{D}_{2}^{\prime}\left(s_{2}^{\prime \prime}\right)>0$, and $s_{2}^{\prime} \stackrel{a}{\longrightarrow} \mathscr{D}_{2}^{\prime}$ obviously exist in the case that \# is $\sqcup$ because $\mathscr{D}_{1}^{\prime}\left(s_{1}^{\prime \prime}\right)>0$. They also exist in the case that \# is $\square$ because, if $s_{2}^{\prime}$ had no a-transition reaching $\mathscr{G}^{\prime}$ (the group composed only of the equivalence class containing $s_{1}^{\prime \prime}$ ) with probability greater than 0 , then all a-transitions of $s_{2}^{\prime}$ would reach $\mathscr{G}^{\prime \prime}=2^{S / \mathscr{B}} \backslash \mathscr{G}^{\prime}$ with probability 1 and hence we would have:

$$
\prod_{s_{1}^{\prime} \stackrel{a}{\longrightarrow} \mathscr{D}_{1}^{\prime}} \mathscr{D}_{1}^{\prime}\left(\bigcup^{\prime \prime} \mathscr{G}^{\prime \prime}\right)<1=\prod_{s_{2}^{\prime} \stackrel{a}{\longrightarrow} \mathscr{D}_{2}^{\prime}} \mathscr{D}_{2}^{\prime}\left(\bigcup \mathscr{G}^{\prime \prime}\right)
$$

i.e., the considered relation $\mathscr{B}$ would not be a $\sqcap$-group-by-group probabilistic bisimulation

We conclude by showing that all the considered $\sim_{\mathrm{B}, \mathrm{m}}$-inspired probabilistic bisimilarities collapse into $\sim \sim_{\mathrm{B}, \mathrm{m}}$ when restricting attention to fully nondeterministic processes. An analogous result holds for their ct-variants.

Theorem 13 Let $(S, A, \longrightarrow)$ be an NPLTS in which the target of each transition is a Dirac distribution. Let $s_{1}, s_{2} \in S$ and $\circ \in\{=, \leq, \geq, \sqcup \sqcap, \sqcup, \sqcap\}$. Then:

$$
s_{1} \sim_{\mathrm{PB}, \mathrm{dis}, \mathrm{m}} s_{2} \Longleftrightarrow s_{1} \sim \mathrm{PB}, \mathrm{gbg}, \mathrm{o}, \mathrm{m}, s_{2} \Longleftrightarrow s_{1} \sim_{\mathrm{B}, \mathrm{m}} s_{2}
$$


Proof Since every multistep transition of this specific NPLTS can reach with probability greater than 0 a single state and hence a single class of any equivalence relation - which are thus reached with probability 1 - the reflexive, symmetric, and transitive closure of a multistep bisimulation is trivially a multistep classdistribution probabilistic bisimulation and a multistep ○-group-by-group probabilistic bisimulation.

\section{B Multistep Variants Inspired by $\sim_{\mathrm{PB}}$}

We start by introducing the multistep variant of $\sim_{\mathrm{PB}}$ and proving that it coincides with $\sim$ PB itself. Given an $\operatorname{NPLTS}(S, A, \longrightarrow)$ in which the transitions of each state have different labels and given $s \in S, \alpha \in A^{*}$, and $S^{\prime} \subseteq S$, we inductively define the multistep probability of reaching a state in $S^{\prime}$ from $s$ via $\alpha$ as follows:

$$
\operatorname{prob}_{\mathrm{m}}\left(s, \alpha, S^{\prime}\right)= \begin{cases}\sum_{s^{\prime} \in S} \mathscr{D}\left(s^{\prime}\right) \cdot \operatorname{prob}_{\mathrm{m}}\left(s^{\prime}, \alpha^{\prime}, S^{\prime}\right) & \text { if } \alpha=a \alpha^{\prime} \text { and } s \stackrel{a}{\longrightarrow} \mathscr{D} \\ 1 & \text { if } \alpha=\varepsilon \text { and } s \in S^{\prime} \\ 0 & \text { if } \alpha=a \alpha^{\prime} \text { and } s \stackrel{a}{\rightarrow}, \text { or } \alpha=\varepsilon \text { and } s \notin S^{\prime}\end{cases}
$$

Definition 17 Let $(S, A, \longrightarrow)$ be an NPLTS in which the transitions of each state have different labels. An equivalence relation $\mathscr{B}$ over $S$ is a $p$-multistep probabilistic bisimulation iff, whenever $\left(s_{1}, s_{2}\right) \in \mathscr{B}$, then for all traces $\alpha \in A^{*}$ and for all equivalence classes $C \in S / \mathscr{B}$ it holds that:

$$
\operatorname{prob}_{\mathrm{m}}\left(s_{1}, \alpha, C\right)=\operatorname{prob}_{\mathrm{m}}\left(s_{2}, \alpha, C\right)
$$

We denote by $\sim_{\mathrm{PB}, \mathrm{pm}}$ the largest p-multistep probabilistic bisimulation.

Theorem 14 Let $(S, A, \longrightarrow)$ be an NPLTS in which the transitions of each state have different labels. Let $s_{1}, s_{2} \in S$. Then:

$$
s_{1} \sim_{\mathrm{PB}, \mathrm{pm}} s_{2} \Longleftrightarrow s_{1} \sim_{\mathrm{PB}} s_{2}
$$

Proof Suppose that $s_{1} \sim_{\mathrm{PB}, \mathrm{pm}} s_{2}$. This means that there exists a p-multistep probabilistic bisimulation $\mathscr{B}$ over $S$ such that $\left(s_{1}, s_{2}\right) \in \mathscr{B}$. As a consequence, we have in particular that, whenever $\left(s_{1}^{\prime}, s_{2}^{\prime}\right) \in \mathscr{B}$, then for all $a \in A$ and $C \in S / \mathscr{B}$ :

$$
\begin{array}{r}
\operatorname{prob}_{\mathrm{m}}\left(s_{1}^{\prime}, a, C\right)=\operatorname{prob}_{\mathrm{m}}\left(s_{2}^{\prime}, a, C\right) \\
\text { Since } \stackrel{a}{\longrightarrow} \text { coincides with } \stackrel{a}{\longrightarrow} \text { and for all } s \in S \text { such that } s \stackrel{a}{\longrightarrow} \mathscr{D} \text { it holds that: } \\
\operatorname{prob}_{\mathrm{m}}(s, a, C)=\sum_{s^{\prime} \in C} \mathscr{D}\left(s^{\prime}\right)=\mathscr{D}(C)
\end{array}
$$

we have that the existence of $s_{1}^{\prime} \stackrel{a}{\longrightarrow} \mathscr{D}_{1}$ implies the existence of $s_{2}^{\prime} \stackrel{a}{\longrightarrow} \mathscr{D}_{2}$ and $\mathscr{D}_{1}(C)=\mathscr{D}_{2}(C)$. In other words, $\mathscr{B}$ is also a probabilistic bisimulation and hence $s_{1} \sim \mathrm{PB} s_{2}$.

Suppose now that $s_{1} \sim \mathrm{PB} s_{2}$. This means that there exists a probabilistic bisimulation $\mathscr{B}$ over $S$ such that $\left(s_{1}, s_{2}\right) \in \mathscr{B}$. We prove that $\mathscr{B}$ is also a p-multistep probabilistic bisimulation, so that $s_{1} \sim \mathrm{PB}, \mathrm{pm} s_{2}$ will follow. Given $s_{1}^{\prime}, s_{2}^{\prime} \in S$ such that $\left(s_{1}^{\prime}, s_{2}^{\prime}\right) \in \mathscr{B}, \alpha \in A^{*}$, and $C \in S / \mathscr{B}$, we proceed by induction on $|\alpha|$ :

- If $|\alpha|=0$, then $s_{1}^{\prime} \stackrel{\alpha}{\Longrightarrow} \delta_{s_{1}^{\prime}}$ and $s_{2}^{\prime} \stackrel{\alpha}{\Longrightarrow} \delta_{s_{2}^{\prime}}$ are the only possible computations from $s_{1}^{\prime}$ and $s_{2}^{\prime}$ labeled with $\alpha$ and it holds that:

$$
\operatorname{prob}_{\mathrm{m}}\left(s_{1}^{\prime}, \alpha, C\right)=\operatorname{prob}_{\mathrm{m}}\left(s_{2}^{\prime}, \alpha, C\right)= \begin{cases}1 & \text { if }\left\{s_{1}^{\prime}, s_{2}^{\prime}\right\} \subseteq C \\ 0 & \text { if }\left\{s_{1}^{\prime}, s_{2}^{\prime}\right\} \cap C=\emptyset\end{cases}
$$

because $\left(s_{1}^{\prime}, s_{2}^{\prime}\right) \in \mathscr{B}$ and $C$ is an equivalence class with respect to $\mathscr{B}$.

- Let $|\alpha|=n \in \mathbb{N}_{>0}$ and suppose that the result holds for all traces of length $n-1$. Assume $\alpha=a \alpha^{\prime}$. Since $\left(s_{1}^{\prime}, s_{2}^{\prime}\right) \in \mathscr{B}$ and $\mathscr{B}$ is a probabilistic bisimulation, for all $C^{\prime} \in S / \mathscr{B}$ it holds that the existence of $s_{1}^{\prime} \stackrel{a}{\longrightarrow} \mathscr{D}_{1}$ implies the existence of $s_{2}^{\prime} \stackrel{a}{\longrightarrow} \mathscr{D}_{2}$ and $\mathscr{D}_{1}\left(C^{\prime}\right)=\mathscr{D}_{2}\left(C^{\prime}\right)$.

Given $s \in S$ such that $s \stackrel{\alpha}{\Longrightarrow}$ with $s \stackrel{a}{\longrightarrow} \mathscr{D}$, it holds that:

$$
\begin{aligned}
\operatorname{prob}_{\mathrm{m}}(s, \alpha, C) & =\sum_{s^{\prime} \in S} \mathscr{D}\left(s^{\prime}\right) \cdot \operatorname{prob}_{\mathrm{m}}\left(s^{\prime}, \alpha^{\prime}, C\right) \\
& =\sum_{C^{\prime} \in S / \mathscr{B}} \sum_{s^{\prime} \in C^{\prime}} \mathscr{D}\left(s^{\prime}\right) \cdot \operatorname{prob}_{\mathrm{m}}\left(s^{\prime}, \alpha^{\prime}, C\right) \\
& =\sum_{C^{\prime} \in S / \mathscr{B}} \sum_{s^{\prime} \in C^{\prime}} \mathscr{D}\left(s^{\prime}\right) \cdot \operatorname{prob}_{\mathrm{m}}\left(s_{C^{\prime}}, \alpha^{\prime}, C\right) \\
& =\sum_{C^{\prime} \in S / \mathscr{B}} \operatorname{prob}_{\mathrm{m}}\left(s_{C^{\prime}}, \alpha^{\prime}, C\right) \cdot \sum_{s^{\prime} \in C^{\prime}} \mathscr{D}\left(s^{\prime}\right) \\
& =\sum_{C^{\prime} \in S / \mathscr{B}} \operatorname{prob}_{\mathrm{m}}\left(s_{C^{\prime}}, \alpha^{\prime}, C\right) \cdot \mathscr{D}\left(C^{\prime}\right)
\end{aligned}
$$


where $s_{C^{\prime}} \in C^{\prime}$ and the factorization of prob ${ }_{\mathrm{m}}\left(s_{C^{\prime}}, \alpha^{\prime}, C\right)$ stems from the application of the induction hypothesis on $\alpha^{\prime}$ to all states of each equivalence class $C^{\prime}$. Since the existence of $s_{1}^{\prime} \stackrel{a}{\longrightarrow} \mathscr{D}_{1}$ implies the existence of $s_{2}^{\prime} \stackrel{a}{\longrightarrow} \mathscr{D}_{2}$ and $\mathscr{D}_{1}\left(C^{\prime}\right)=\mathscr{D}_{2}\left(C^{\prime}\right)$ for all $C^{\prime} \in S / \mathscr{B}$ - remember that the quantification over $C^{\prime}$ can be equivalently anticipated or postponed in the absence of internal nondeterminism - we derive that:

$$
\operatorname{prob}_{\mathrm{m}}\left(s_{1}^{\prime}, \alpha, C\right)=\operatorname{prob}_{\mathrm{m}}\left(s_{2}^{\prime}, \alpha, C\right)
$$

When considering an arbitrary NPLTS $(S, A, \longrightarrow)$, internal nondeterminism comes into play and hence there might be several computations labeled with the same trace belonging to different resolutions of nondeterminism. In that case, their multistep probabilities have to be kept separate, otherwise they would be summed up like in the case of reactive probabilistic processes.

Since preserving the connection between each computation and the resolution of nondeterminism to which it belongs is important to define a $\sim \mathrm{PB}, \mathrm{m}$-inspired multistep variant of $\sim_{\mathrm{PB}, \mathrm{dis}}$, we formalize below the notion of resolution. We call resolution of a state $s$ of an NPLTS $\mathscr{L}$ the result of a possible way of resolving nondeterminism starting from $s$. A resolution is a tree-like structure, whose branching points are probabilistic choices corresponding to target distributions of transitions. This is obtained by unfolding from $s$ the graph structure underlying $\mathscr{L}$ and by selecting at each reached state at most one transition - deterministic scheduler - or a convex combination of equally labeled transitions - randomized scheduler - among all the transitions in $\mathscr{L}$ departing from that state. A resolution of $s$ can be formalized as an NPLTS $\mathscr{Z}$ rooted at a state $z_{s}$ corresponding to $s$, in which every state has at most one outgoing transition, so that function $p r o b_{\mathrm{m}}$ can be safely applied.

Definition 18 Let $\mathscr{L}=(S, A, \longrightarrow)$ be an NPLTS and $s \in S$. An NPLTS $\mathscr{Z}=(Z, A, \longrightarrow \mathscr{Z})$ is a resolution of $s$ obtained via a deterministic scheduler iff there exists a state correspondence function corr $: Z \rightarrow S$ such that $s=\operatorname{corr}\left(z_{s}\right)$, for some $z_{s} \in Z$, and for all $z \in Z$ it holds that:

- If $z \stackrel{a}{\longrightarrow} \mathscr{Z} \mathscr{D}$, then $\operatorname{corr}(z) \stackrel{a}{\longrightarrow} \mathscr{D}^{\prime}$ with corr being injective over $\operatorname{supp}(\mathscr{D})$ and $\mathscr{D}\left(z^{\prime}\right)=\mathscr{D}^{\prime}\left(\operatorname{corr}\left(z^{\prime}\right)\right)$ for all $z^{\prime} \in \operatorname{supp}(\mathscr{D})$.

- If $z \stackrel{a_{1}}{\longrightarrow} \mathscr{Z} \mathscr{D}_{1}$ and $z \stackrel{a_{2}}{\longrightarrow} \mathscr{Z} \mathscr{D}_{2}$, then $a_{1}=a_{2}$ and $\mathscr{D}_{1}=\mathscr{D}_{2}$.

We denote by $\operatorname{Res}(s)$ the set of resolutions of $s$.

On the basis of the notion above, we provide a $\sim \mathrm{PB}$,pm-inspired definition of $\sim$ PB,dis and show that it coincides with $\sim$ PB, dis itself. The ct-variant of the $\sim_{\mathrm{PB}, \mathrm{pm}}$-inspired equivalence can be defined similarly by relying on resolutions obtained from randomized schedulers, and satisfies an analogous property with respect to the original one-step ct-equivalence.

Definition 19 Let $(S, A, \longrightarrow)$ be an NPLTS. An equivalence relation $\mathscr{B}$ over $S$ is a $p$-multistep class-distribution probabilistic bisimulation iff, whenever $\left(s_{1}, s_{2}\right) \in \mathscr{B}$, then for all traces $\alpha \in A^{*}$ it holds that for each resolution $\mathscr{Z}_{1} \in \operatorname{Res}\left(s_{1}\right)$ there exists a resolution $\mathscr{Z}_{2} \in \operatorname{Res}\left(s_{2}\right)$ such that for all equivalence classes $C \in S / \mathscr{B}$ :

$$
\operatorname{prob}_{\mathrm{m}}\left(z_{s_{1}}, \alpha, \operatorname{corr}_{\mathscr{Z}_{1}}^{-1}(C)\right)=\operatorname{prob}_{\mathrm{m}}\left(z_{s_{2}}, \alpha, \operatorname{corr}_{\mathscr{Z}_{2}}^{-1}(C)\right)
$$

We denote by $\sim$ PB,dis.pm the largest p-multistep class-distribution probabilistic bisimulation.

Theorem 15 Let $(S, A, \longrightarrow)$ be an NPLTS and $s_{1}, s_{2} \in S$. Then:

$$
s_{1} \sim \sim_{\mathrm{PB}, \mathrm{dis}, \mathrm{pm}} s_{2} \Longleftrightarrow s_{1} \sim_{\mathrm{PB}, \mathrm{dis}} s_{2}
$$

Proof Suppose that $s_{1} \sim \mathrm{PB}$,dis,pm $s_{2}$. This means that there exists a p-multistep class-distribution probabilistic bisimulation $\mathscr{B}$ over $S$ such that $\left(s_{1}, s_{2}\right) \in \mathscr{B}$. As a consequence, we have in particular that, whenever $\left(s_{1}^{\prime}, s_{2}^{\prime}\right) \in \mathscr{B}$, then for all $a \in A$ it holds that for each $\mathscr{Z}_{1} \in \operatorname{Res}\left(s_{1}\right)$ there exists $\mathscr{Z}_{2} \in \operatorname{Res}\left(s_{2}\right)$ such that for all $C \in S / \mathscr{B}:$

$$
\begin{gathered}
\operatorname{prob}_{\mathrm{m}}\left(z_{s_{1}}, \text { a, } \operatorname{corr}_{\mathscr{Z}_{1}}^{-1}(C)\right)=\operatorname{prob}_{\mathrm{m}}\left(z_{s_{2}}, \text { a, } \operatorname{corr}_{\mathscr{Z}_{2}}^{-1}(C)\right) \\
\text { Since } \stackrel{a}{\Longrightarrow} \text { coincides with } \stackrel{a}{\longrightarrow} \text { and for all } s \in S \text { and } \mathscr{Z} \in \operatorname{Res}(s) \text { it holds that: } \\
\operatorname{prob}_{\mathrm{m}}\left(z_{s}, a, \operatorname{corr}_{\mathscr{Z}}^{-1}(C)\right)=\sum_{z_{s^{\prime}} \in \operatorname{corr}_{\mathscr{Z}}^{-1}(C)} \mathscr{D}\left(z_{s^{\prime}}\right)=\mathscr{D}\left(\operatorname{corr}_{\mathscr{Z}}^{-1}(C)\right)
\end{gathered}
$$

we have that for each $s_{1}^{\prime} \stackrel{a}{\longrightarrow} \mathscr{D}_{1}$ there exists $s_{2}^{\prime} \stackrel{a}{\longrightarrow} \mathscr{D}_{2}$ such that, for all $C \in S / \mathscr{B}, \mathscr{D}_{1}(C)=\mathscr{D}_{2}(C)$. In other words, $\mathscr{B}$ is also a class-distribution probabilistic bisimulation and hence $s_{1} \sim_{\mathrm{PB}, \mathrm{dis}} s_{2}$.

Suppose now that $s_{1} \sim \mathrm{PB}$, dis $s_{2}$. This means that there exists a class-distribution probabilistic bisimulation $\mathscr{B}$ over $S$ such that $\left(s_{1}, s_{2}\right) \in \mathscr{B}$. We prove that $\mathscr{B}$ is also a p-multistep class-distribution probabilistic bisimulation, so that $s_{1} \sim \mathrm{PB}$,dis,pm $s_{2}$ will follow. Given $s_{1}^{\prime}, s_{2}^{\prime} \in S$ such that $\left(s_{1}^{\prime}, s_{2}^{\prime}\right) \in \mathscr{B}$ and $\alpha \in A^{*}$, we proceed by induction on $|\alpha|$ : 
- If $|\alpha|=0$, then $z_{s_{1}^{\prime}} \stackrel{\alpha}{\Longrightarrow} \delta_{z_{s_{1}^{\prime}}}$ and $z_{s_{2}^{\prime}} \stackrel{\alpha}{\Longrightarrow} \delta_{z_{s_{2}}}$ are the only possible computations labeled with $\alpha$ in any resolution $\mathscr{Z}_{1} \in \operatorname{Res}\left(s_{1}^{\prime}\right)$ and any resolution $\mathscr{Z}_{2} \in \operatorname{Res}\left(s_{2}^{\prime}\right)$, respectively, and for all $C \in S / \mathscr{B}$ it holds that:

$$
\operatorname{prob}_{\mathrm{m}}\left(z_{s_{1}^{\prime}}, \alpha, \operatorname{corr}_{\mathscr{Z}_{1}}^{-1}(C)\right)=\operatorname{prob}_{\mathrm{m}}\left(z_{s_{2}^{\prime}}, \alpha, \operatorname{corr}_{\mathscr{Z}_{2}}^{-1}(C)\right)= \begin{cases}1 & \text { if }\left\{s_{1}^{\prime}, s_{2}^{\prime}\right\} \subseteq C \\ 0 & \text { if }\left\{s_{1}^{\prime}, s_{2}^{\prime}\right\} \cap C=\emptyset\end{cases}
$$

because $\left(s_{1}^{\prime}, s_{2}^{\prime}\right) \in \mathscr{B}$ and $C$ is an equivalence class with respect to $\mathscr{B}$.

- Let $|\alpha|=n \in \mathbb{N}_{>0}$ and suppose that the result holds for all traces of length $n-1$. Assume $\alpha=a \alpha^{\prime}$. Since $\left(s_{1}^{\prime}, s_{2}^{\prime}\right) \in \mathscr{B}$ and $\mathscr{B}$ is a class-distribution probabilistic bisimulation, it holds that for each $s_{1}^{\prime} \stackrel{a}{\longrightarrow} \mathscr{D}_{1}$ there exists $s_{2}^{\prime} \stackrel{a}{\longrightarrow} \mathscr{D}_{2}$ such that, for all $C \in S / \mathscr{B}, \mathscr{D}_{1}(C)=\mathscr{D}_{2}(C)$.

Given $s \in S$ such that $z_{s} \stackrel{\alpha}{\Longrightarrow}$ with $z_{s} \stackrel{a}{\longrightarrow} \mathscr{D}$ in a resolution $\mathscr{Z} \in \operatorname{Res}(s)$, for all $C \in S / \mathscr{B}$ it holds that: $\operatorname{prob}_{\mathrm{m}}\left(z_{s}, \alpha, \operatorname{corr}_{\mathscr{Z}}^{-1}(C)\right)=\sum_{z_{s^{\prime}} \in Z} \mathscr{D}\left(z_{s^{\prime}}\right) \cdot \operatorname{prob}_{\mathrm{m}}\left(z_{s^{\prime}}, \alpha^{\prime}, \operatorname{corr}_{\mathscr{Z}}^{-1}(C)\right)$

$$
\begin{aligned}
& =\sum_{C^{\prime} \in S / \mathscr{B}} \sum_{z_{s^{\prime}} \in \operatorname{corr}_{\mathscr{Z}}^{-1}\left(C^{\prime}\right)} \mathscr{D}\left(z_{s^{\prime}}\right) \cdot \operatorname{prob}_{\mathrm{m}}\left(z_{s^{\prime}}, \alpha^{\prime}, \operatorname{corr}_{\mathscr{Z}}^{-1}(C)\right) \\
& =\sum_{C^{\prime} \in S / \mathscr{B}} \sum_{z_{s^{\prime}} \in \operatorname{corr}_{\mathscr{Z}}^{-1}\left(C^{\prime}\right)} \mathscr{D}\left(z_{s^{\prime}}\right) \cdot \operatorname{prob}_{\mathrm{m}}\left(z_{S_{C^{\prime}}}, \alpha^{\prime}, \operatorname{corr}_{\mathscr{Z}}^{-1}(C)\right) \\
& =\sum_{C^{\prime} \in S / \mathscr{B}} \operatorname{prob}_{\mathrm{m}}\left(z_{C_{C^{\prime}}}, \alpha^{\prime}, \operatorname{corr}_{\mathscr{Z}}^{-1}(C)\right) \cdot \sum_{z_{s^{\prime}} \in \operatorname{corr}_{\mathscr{Z}}^{-1}\left(C^{\prime}\right)} \mathscr{D}\left(z_{s^{\prime}}\right) \\
& =\sum_{C^{\prime} \in S / \mathscr{B}} \operatorname{prob}_{\mathrm{m}}\left(z_{S_{C^{\prime}}}, \alpha^{\prime}, \operatorname{corr}_{\mathscr{Z}}^{-1}(C)\right) \cdot \mathscr{D}\left(\operatorname{corr}_{\mathscr{Z}}^{-1}\left(C^{\prime}\right)\right)
\end{aligned}
$$

where $s_{C^{\prime}} \in C^{\prime}$ and the factorization of $\operatorname{prob}_{\mathrm{m}}\left(z_{S_{C^{\prime}}}, \alpha^{\prime}, \operatorname{corr}_{\mathscr{Z}}^{-1}(C)\right)$ stems from the application of the induction hypothesis on $\alpha^{\prime}$ to all states of each equivalence class $C^{\prime}$. Since for each $s_{1}^{\prime} \stackrel{a}{\longrightarrow} \mathscr{D}_{1}$ there exists $s_{2}^{\prime} \stackrel{a}{\longrightarrow} \mathscr{D}_{2}$ such that, for all $C^{\prime} \in S / \mathscr{B}, \mathscr{D}_{1}\left(C^{\prime}\right)=\mathscr{D}_{2}\left(C^{\prime}\right)$, we derive that for each $\mathscr{Z}_{1} \in$ Res $\left(s_{1}^{\prime}\right)$ there exists $\mathscr{Z}_{2} \in \operatorname{Res}\left(s_{2}^{\prime}\right)$ such that for all $C \in S / \mathscr{B}$ :

$$
\operatorname{prob}_{\mathrm{m}}\left(z_{s_{1}^{\prime}}, \alpha, \operatorname{corr}_{\mathscr{Z}}^{-1}(C)\right)=\operatorname{prob}_{\mathrm{m}}\left(z_{s_{2}^{\prime}}, \alpha, \operatorname{corr}_{\mathscr{Z}}^{-1}(C)\right)
$$

Using the notion of resolution, we can also provide a $\sim_{\mathrm{PB}, \mathrm{pm}}$-inspired definition of each of the six groupby-group probabilistic bisimilarities. The ct-variants of the six $\sim_{\mathrm{PB}, \mathrm{pm}}$-inspired group-by-group probabilistic bisimilarities can be defined similarly by relying on resolutions obtained from randomized schedulers.

Definition 20 Let $(S, A, \longrightarrow)$ be an NPLTS and $\bowtie \in\{=, \leq, \geq\}$. An equivalence relation $\mathscr{B}$ over $S$ is a p-multistep $\bowtie$-group-by-group probabilistic bisimulation iff, whenever $\left(s_{1}, s_{2}\right) \in \mathscr{B}$, then for all traces $\alpha \in A^{*}$ and for all groups of equivalence classes $\mathscr{G} \in 2^{S / \mathscr{B}}$ it holds that for each resolution $\mathscr{Z}_{1} \in \operatorname{Res}\left(s_{1}\right)$ there exists a resolution $\mathscr{Z}_{2} \in \operatorname{Res}\left(s_{2}\right)$ such that:

$$
\operatorname{prob}_{\mathrm{m}}\left(z_{s_{1}}, \alpha, \operatorname{corr}_{\mathscr{Z}_{1}}^{-1}(\bigcup \mathscr{G})\right) \bowtie \operatorname{prob}_{\mathrm{m}}\left(z_{s_{2}}, \alpha, \operatorname{corr}_{\mathscr{Z}_{2}}^{-1}(\bigcup \mathscr{G})\right)
$$

We denote by $\sim$ PB,gbg, $\bowtie$,pm the largest p-multistep $\bowtie$-group-by-group probabilistic bisimulation.

Definition 21 Let $(S, A, \longrightarrow)$ be an NPLTS. An equivalence relation $\mathscr{B}$ over $S$ is a $p$-multistep $\sqcup \sqcap$-groupby-group probabilistic bisimulation iff, whenever $\left(s_{1}, s_{2}\right) \in \mathscr{B}$, then for all traces $\alpha \in A^{*}$ and for all groups of equivalence classes $\mathscr{G} \in 2^{S / \mathscr{B}}$ it holds that $s_{1} \stackrel{\alpha}{\Longrightarrow}$ iff $s_{2} \stackrel{\alpha}{\Longrightarrow}$ and:

$$
\begin{aligned}
& \bigsqcup_{\mathscr{Z}_{1} \in \operatorname{Res}\left(s_{1}\right) \text { s.t. } z_{s_{1}} \stackrel{\alpha}{\Longrightarrow}} \operatorname{prob}_{\mathrm{m}}\left(z_{s_{1}}, \alpha, \operatorname{corr}_{\mathscr{Z}_{1}}^{-1}(\bigcup \mathscr{G})\right)=\underset{\mathscr{Z}_{2} \in \operatorname{Res}\left(s_{2}\right) \text { s.t. } z_{s_{2}}}{\longrightarrow} \operatorname{prob}_{\mathrm{m}}\left(z_{s_{2}}, \alpha, \operatorname{corr}_{\mathscr{Z}_{2}}^{-1}(\bigcup \mathscr{G})\right) \\
& \prod_{\mathscr{Z}_{1} \in \operatorname{Res}\left(s_{1}\right) \text { s.t. } z_{s_{1}} \stackrel{\alpha}{\Longrightarrow}} \operatorname{prob}_{\mathrm{m}}\left(z_{s_{1}}, \alpha, \operatorname{corr}_{\mathscr{Z}_{1}}^{-1}(\bigcup \mathscr{G})\right)=\prod_{\mathscr{Z}_{2} \in \operatorname{Res}\left(s_{2}\right) \text { s.t. } z_{s_{2}} \stackrel{\alpha}{\Longrightarrow}} \operatorname{prob}_{\mathrm{m}}\left(z_{s_{2}}, \alpha, \operatorname{corr}_{\mathscr{Z}_{2}}^{-1}(\bigcup \mathscr{G})\right)
\end{aligned}
$$

We denote by $\sim_{\mathrm{PB}, \mathrm{gbg}, \sqcup \sqcap, \mathrm{pm}}$ the largest p-multistep $\sqcup \sqcap$-group-by-group probabilistic bisimulation.

Definition 22 Let $(S, A, \longrightarrow)$ be an NPLTS and \# $\in\{\sqcup, \Pi\}$. An equivalence relation $\mathscr{B}$ over $S$ is a p-multistep \#-group-by-group probabilistic bisimulation iff, whenever $\left(s_{1}, s_{2}\right) \in \mathscr{B}$, then for all traces $\alpha \in A^{*}$ and for all groups of equivalence classes $\mathscr{G} \in 2^{S / \mathscr{B}}$ it holds that $s_{1} \stackrel{\alpha}{\Longrightarrow}$ iff $s_{2} \stackrel{\alpha}{\Longrightarrow}$ and:

$$
\underset{\mathscr{Z}_{1} \in \operatorname{Res}\left(s_{1}\right) \text { s.t. } z_{s_{1}} \stackrel{\alpha}{\Longrightarrow}}{\operatorname{prob}} \operatorname{mrob}_{\mathrm{m}}\left(z_{s_{1}}, \alpha, \operatorname{corr}_{\mathscr{Z}_{1}}^{-1}(\bigcup \mathscr{G})\right)=\underset{\mathscr{Z}_{2} \in \operatorname{Res}\left(s_{2}\right) \text { s.t. } z_{s_{2}} \stackrel{\alpha}{\Longrightarrow}}{\#} \operatorname{prob}_{\mathrm{m}}\left(z_{s_{2}}, \alpha, \operatorname{corr}_{\mathscr{Z}_{2}}^{-1}(\bigcup \mathscr{G})\right)
$$

We denote by $\sim_{\mathrm{PB} \text {,gbg,\#,pm }}$ the largest p-multistep \#-group-by-group probabilistic bisimulation.

The six $\sim$ PB,pm-inspired group-by-group probabilistic bisimilarities can be alternatively defined without making explicit use of the notion of resolution. Given $s \in S, \alpha \in A^{*}$, and $S^{\prime} \subseteq S$, we inductively define the set of multistep probabilities of reaching a state in $S^{\prime}$ from $s$ via $\alpha$ as follows: 


$$
\text { probset }_{\mathrm{m}}\left(s, \alpha, S^{\prime}\right)= \begin{cases}\bigcup_{a} \underset{\longrightarrow}{\longrightarrow}\left\{\sum_{s^{\prime} \in S} \mathscr{D}\left(s^{\prime}\right) \cdot p_{s^{\prime}} \mid p_{s^{\prime}} \in \text { probset }_{\mathrm{m}}\left(s^{\prime}, \alpha^{\prime}, S^{\prime}\right)\right\} & \text { if } \alpha=a \alpha^{\prime} \text { and } s \stackrel{a}{\longrightarrow} \\ \{1\} & \text { if } \alpha=\varepsilon \text { and } s \in S^{\prime} \\ \{0\} & \text { if } \alpha=a \alpha^{\prime} \text { and } s \stackrel{a}{A}\end{cases}
$$

Since $\operatorname{probset}_{\mathrm{m}}\left(s, \alpha, S^{\prime}\right)=\left\{\operatorname{prob}_{\mathrm{m}}\left(z_{s}, \alpha, \operatorname{corr}_{\mathscr{Z}}^{-1}\left(S^{\prime}\right)\right) \mid \mathscr{Z} \in \operatorname{Res}(s)\right\}$, it is easy to see that in Defs. 20 to 22 we could have used $\operatorname{probset}_{\mathrm{m}}\left(s_{i}, \alpha, \bigcup \mathscr{G}\right)$ in place of $\operatorname{prob}_{\mathrm{m}}\left(z_{s_{i}}, \alpha, \operatorname{corr}_{\mathscr{Z}_{i}}^{-1}(\bigcup \mathscr{G})\right)$ for $i=1,2$. This is not possible in Def. 19 because the use of probset $t_{\mathrm{m}}$ causes the connection between each computation and the resolution to which it belongs to be broken.

Each of the six $\sim \mathrm{PB}, \mathrm{pm}$-inspired group-by-group probabilistic bisimilarities is contained in the corresponding original one-step equivalence. The ct-variants of the six $\sim \mathrm{PB}, \mathrm{pm}$-inspired group-by-group probabilistic bisimilarities satisfy an analogous inclusion property with respect to the original one-step ct-equivalences.

Theorem 16 Let $(S, A, \longrightarrow)$ be an NPLTS, $s_{1}, s_{2} \in S$, and $\circ \in\{=, \leq, \geq, \sqcup \sqcap, \sqcup, \sqcap\}$. Then:

$$
s_{1} \sim \text { PB,gbg,o,pm } s_{2} \Longrightarrow s_{1} \sim \text { PB,gbg,o } s_{2}
$$

Proof Let $\bowtie \in\{=, \leq, \geq\}$ and suppose that $s_{1} \sim_{\mathrm{PB}, \mathrm{gbg}, \bowtie, \mathrm{pm}} s_{2}$. This means that there exists a p-multistep $\bowtie$-group-by-group probabilistic bisimulation $\mathscr{B}$ over $S$ such that $\left(s_{1}, s_{2}\right) \in \mathscr{B}$. As a consequence, we have in particular that, whenever $\left(s_{1}^{\prime}, s_{2}^{\prime}\right) \in \mathscr{B}$, then for all $a \in A$ and $\mathscr{G} \in 2^{S / \mathscr{B}}$ it holds that for each $\mathscr{Z}_{1} \in \operatorname{Res}\left(s_{1}^{\prime}\right)$ there exists $\mathscr{Z}_{2} \in \operatorname{Res}\left(s_{2}^{\prime}\right)$ such that:

$$
\begin{aligned}
& \text { Since } \stackrel{a}{\longrightarrow} \text { coincides with } \stackrel{a}{\longrightarrow} \text { and for all } s \in S \text { and } \mathscr{Z} \in \operatorname{Res}(s) \text { it holds that: } \\
& \operatorname{prob}_{\mathrm{m}}\left(z_{s}, a, \operatorname{corr}_{\mathscr{Z}}^{-1}(\bigcup \mathscr{G})\right)=\sum_{z_{s^{\prime}} \in \operatorname{corr}_{\mathscr{L}}^{-1}(\bigcup \mathscr{G})} \mathscr{D}\left(z_{s^{\prime}}\right)=\mathscr{D}\left(\operatorname{corr}_{\mathscr{L}}^{-1}(\bigcup \mathscr{G})\right)
\end{aligned}
$$

we have that for each $s_{1}^{\prime} \stackrel{a}{\longrightarrow} \mathscr{D}_{1}$ there exists $s_{2}^{\prime} \stackrel{a}{\longrightarrow} \mathscr{D}_{2}$ such that $\mathscr{D}_{1}(\bigcup \mathscr{G}) \bowtie \mathscr{D}_{2}(\bigcup \mathscr{G})$. In other words, $\mathscr{B}$ is also a $\bowtie$-group-by-group probabilistic bisimulation and hence $s_{1} \sim \mathrm{PB}, \mathrm{gbg}, \bowtie s_{2}$.

Suppose now that $s_{1} \sim$ PB,gbg, $\sqcap$, pm $s_{2}$. This means that there exists a p-multistep $\sqcup П$-group-by-group probabilistic bisimulation $\mathscr{B}$ over $S$ such that $\left(s_{1}, s_{2}\right) \in \mathscr{B}$. As a consequence, we have in particular that, whenever $\left(s_{1}^{\prime}, s_{2}^{\prime}\right) \in \mathscr{B}$, then for all $a \in A$ and $\mathscr{G} \in 2^{S / \mathscr{B}}$ it holds that $s_{1}^{\prime} \stackrel{a}{\Longrightarrow}$ iff $s_{2}^{\prime} \stackrel{a}{\Longrightarrow}$ and:

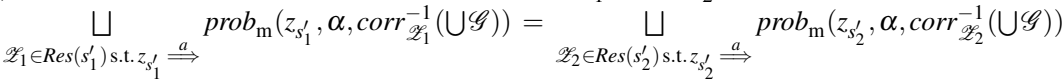

$$
\begin{aligned}
& \prod_{\mathscr{Z}_{1} \in \operatorname{Res}\left(s_{1}^{\prime}\right) \text { s.t. } z_{s_{1}^{\prime}} \stackrel{a}{\Longrightarrow}} \operatorname{prob}_{\mathrm{m}}\left(z_{s_{1}^{\prime}}, \alpha, \operatorname{corr}_{\mathscr{L}_{1}}^{-1}(\bigcup \mathscr{G})\right)=\prod_{\mathscr{Z}_{2} \in \operatorname{Res}\left(s_{2}^{\prime}\right) \text { s.t. } z_{s_{2}^{\prime}} \stackrel{a}{\Longrightarrow}} \operatorname{prob}_{\mathrm{m}}\left(z_{s_{2}^{\prime}}, \alpha, \operatorname{corr}_{\mathscr{Z}_{2}}^{-1}(\bigcup \mathscr{G})\right) \\
& \operatorname{prob}_{\mathrm{m}}\left(z_{s}, a, \operatorname{corr}_{\mathscr{Z}}^{-1}(\bigcup \mathscr{G})\right)=\sum_{z_{s^{\prime}} \in \operatorname{corr}_{\mathscr{L}}^{-1}(\bigcup \mathscr{G})} \mathscr{D}\left(z_{s^{\prime}}\right)=\mathscr{D}\left(\operatorname{corr}_{\mathscr{Z}}^{-1}(\bigcup \mathscr{G})\right)
\end{aligned}
$$

we have that $s_{1}^{\prime} \stackrel{a}{\longrightarrow}$ iff $s_{2}^{\prime} \stackrel{a}{\longrightarrow}$ and:

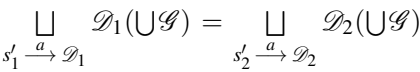

$$
\begin{aligned}
& \prod_{s_{1}^{\prime} \stackrel{a}{\longrightarrow} \mathscr{D}_{1}} \mathscr{D}_{1}(\bigcup \mathscr{G})=\prod_{s_{2}^{\prime} \stackrel{a}{\longrightarrow} \mathscr{D}_{2}} \mathscr{D}_{2}(\bigcup \mathscr{G})
\end{aligned}
$$

In other words, $\mathscr{B}$ is also a $\sqcup \sqcap$-group-by-group probabilistic bisimulation and hence $s_{1} \sim \mathrm{PB}$, gbg. $\sqcup \sqcap s_{2}$. Finally, the proof that $s_{1} \sim \mathrm{PB}, \mathrm{gbg}, \#, \mathrm{pm} s_{2}$ implies $s_{1} \sim \mathrm{PB}, \mathrm{gbg}, \# s_{2}$ for $\# \in\{\sqcup, \sqcap\}$ is similar to the proof that $s_{1} \sim$ PB,gbg, $\sqcup \sqcap, \mathrm{pm} s_{2}$ implies $s_{1} \sim$ PB,gbg, $\sqcup \sqcap s_{2}$.

Unlike Thm. 15, the reverse implication of Thm. 16 does not hold in general. For example, in Fig. 7 we have that $s_{1} \sim_{\mathrm{PB}, \mathrm{gbg},=} s_{2}$ but $s_{1} \chi_{\mathrm{PB}, \mathrm{gbg},=\text {,pm }} s_{2}$ because, for $\alpha=a b c$ and $\mathscr{G}$ containing all the states with no outgoing transitions, it turns out that the multistep probability of reaching $\mathscr{G}$ via $\alpha$ in the maximal resolution of $s_{1}$ starting with the rightmost $a$-transition - which is $0.1 \cdot 0.7+0.9 \cdot 0.6=0.61-$ is not matched by any of the multistep probabilities of reaching $\mathscr{G}$ via $\alpha$ in the three maximal resolutions of $s_{2}$ starting with the three $a$-transitions - which are $0.8 \cdot 0.7+0.2 \cdot 0.6=0.68,0.1 \cdot 0.7=0.07$, and $0.9 \cdot 0.6=0.54$.

We conclude by showing that all the considered $\sim_{\mathrm{PB}, \mathrm{pm}}$-inspired probabilistic bisimilarities collapse into $\sim$ PB,pm when restricting attention to reactive probabilistic processes. An analogous result holds for their ct-variants.

Theorem 17 Let $(S, A, \longrightarrow)$ be an NPLTS in which the transitions of each state have different labels. Let $s_{1}, s_{2} \in S$ and $\circ \in\{=, \leq, \geq, \sqcup \sqcap, \sqcup, \sqcap\}$. Then:

$$
s_{1} \sim \text { PB,dis,pm } s_{2} \Longleftrightarrow s_{1} \sim \mathrm{PB}, \mathrm{gbg}, \circ, \mathrm{pm} s_{2} \Longleftrightarrow s_{1} \sim \mathrm{PB}, \mathrm{pm} s_{2}
$$




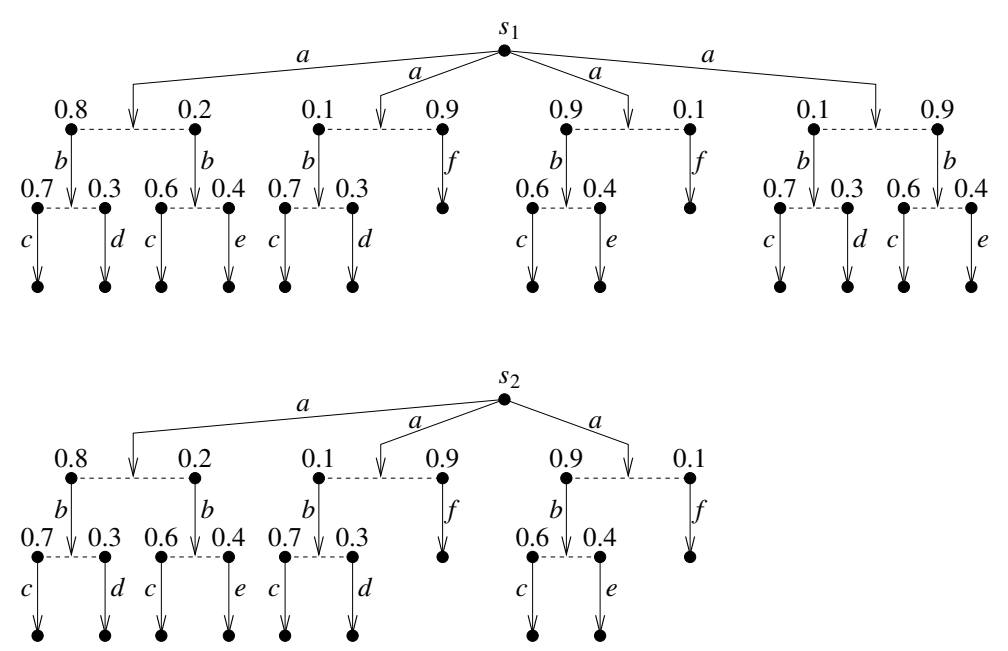

Fig. 7 Two models related by $\sim_{\mathrm{PB}, \mathrm{gbg},=}$ that are distinguished by $\sim_{\mathrm{PB}, \mathrm{gbg},=\text {,pm }}$

Proof Since every state of this specific NPLTS has at most one transition labeled with a certain action, a p-multistep probabilistic bisimulation is trivially a p-multistep class-distribution probabilistic bisimulation and a p-multistep ०-group-by-group probabilistic bisimulation.

\section{References}

1. A. Aziz, V. Singhal, F. Balarin, R.K. Brayton, and A.L. Sangiovanni-Vincentelli. It usually works: The temporal logic of stochastic systems. In Proc. of the 7th Int. Conf. on Computer Aided Verification (CAV 1995), volume 939 of LNCS, pages 155-165. Springer, 1995.

2. C. Baier and M. Kwiatkowska. Domain equations for probabilistic processes. Mathematical Structures in Computer Science, 10:665-717, 2000.

3. F. Bartels, A. Sokolova, and E.P. de Vink. A hierarchy of probabilistic system types. Theoretical Computer Science, 327:3-22, 2004.

4. J. van Benthem. Modal Correspondence Theory. PhD Thesis, 1976.

5. M. Bernardo, R. De Nicola, and M. Loreti. A uniform framework for modeling nondeterministic, probabilistic, stochastic, or mixed processes and their behavioral equivalences. Information and Computation, 225:29-82, 2013.

6. M. Bernardo, R. De Nicola, and M. Loreti. Group-by-group probabilistic bisimilarities and their logical characterizations. In Proc. of the 8th Int. Symp. on Trustworthy Global Computing (TGC 2013), volume 8358 of $L N C S$, pages 315-330. Springer, 2013.

7. A. Bianco and L. de Alfaro. Model checking of probabilistic and nondeterministic systems. In Proc. of the 15th Int. Conf. on Foundations of Software Technology and Theoretical Computer Science (FSTTCS 1995), volume 1026 of LNCS, pages 499-513. Springer, 1995.

8. M.C. Browne, E.M. Clarke, and O. Grümberg. Characterizing finite Kripke structures in propositional temporal logic. Theoretical Computer Science, 59:115-131, 1988.

9. S. Cattani, R. Segala, M. Kwiatkowska, and G. Norman. Stochastic transition systems for continuous state spaces and non-determinism. In Proc. of the 8th Int. Conf. on Foundations of Software Science and Computation Structures (FOSSACS 2005), volume 3441 of LNCS, pages 125-139. Springer, 2005.

10. C. Cîrstea, A. Kurz, D. Pattinson, L. Schröder, and Y. Venema. Modal logics are coalgebraic. Computer Journal, 54:31-41, 2011.

11. E.M. Clarke, E.A. Emerson, and A.P. Sistla. Automatic verification of finite-state concurrent systems using temporal logic specifications. ACM Trans. on Programming Languages and Systems, 8:244-263, 1986.

12. S. Crafa and F. Ranzato. A spectrum of behavioral relations over LTSs on probability distributions. In Proc. of the 22nd Int. Conf. on Concurrency Theory (CONCUR 2011), volume 6901 of LNCS, pages 124-139. Springer, 2011. 
13. P.R. D'Argenio, N. Wolovick, P. Sanchez Terraf, and P. Celayes. Nondeterministic labeled Markov processes: Bisimulations and logical characterizations. In Proc. of the 6th Int. Conf. on the Quantitative Evaluation of Systems (QEST 2009), pages 11-20. IEEE-CS Press, 2009.

14. L. de Alfaro, R. Majumdar, V. Raman, and M. Stoelinga. Game refinement relations and metrics. Logical Methods in Computer Science, 4(3:7):1-28, 2008.

15. R. De Nicola and F. Vaandrager. Three logics for branching bisimulation. Journal of the ACM, 42:458487, 1995.

16. C. Derman. Finite State Markovian Decision Processes. Academic Press, 1970.

17. J. Desharnais, A. Edalat, and P. Panangaden. Bisimulation for labelled Markov processes. Information and Computation, 179:163-193, 2002.

18. J. Desharnais, V. Gupta, R. Jagadeesan, and P. Panangaden. Approximating labelled Markov processes. Information and Computation, 184:160-200, 2003.

19. J. Desharnais, V. Gupta, R. Jagadeesan, and P. Panangaden. Weak bisimulation is sound and complete for pCTL*. Information and Computation, 208:203-219, 2010.

20. M. Giry. A categorical approach to probability theory. In Proc. of the Int. Conf. on Categorical Aspects of Topology and Analysis, volume 915 of $L N M$, pages 68-85. Springer, 1981.

21. R.J. van Glabbeek, S.A. Smolka, and B. Steffen. Reactive, generative and stratified models of probabilistic processes. Information and Computation, 121:59-80, 1995.

22. R. Goldblatt. Deduction systems for coalgebras over measurable spaces. Journal of Logic and Computation, 20:1069-1100, 2010.

23. H. Hansson and B. Jonsson. A calculus for communicating systems with time and probabilities. In Proc. of the 11th IEEE Real-Time Systems Symp. (RTSS 1990), pages 278-287. IEEE-CS Press, 1990.

24. H. Hansson and B. Jonsson. A logic for reasoning about time and reliability. Formal Aspects of Computing, 6:512-535, 1994

25. M. Hennessy. Exploring probabilistic bisimulations, part I. Formal Aspects of Computing, 24:749-768, 2012.

26. M. Hennessy and R. Milner. Algebraic laws for nondeterminism and concurrency. Journal of the ACM, 32:137-162, 1985 .

27. H. Hermanns, A. Parma, R. Segala, B. Wachter, and L. Zhang. Probabilistic logical characterization. Information and Computation, 209:154-172, 2011.

28. G.E. Hughes and M.J. Creswell. An Introduction to Modal Logic. Methuen, 1977.

29. H. Jifeng, K. Seidel, and A. McIver. Probabilistic models for the guarded command language. Science of Computer Programming, 28:171-192, 1997.

30. R.M. Keller. Formal verification of parallel programs. Communications of the ACM, 19:371-384, 1976.

31. B. Klin. Coalgebraic modal logic beyond sets. In Proc. of the 23rd Int. Conf. on the Mathematical Foundations of Programming Semantics (MFPS 2007), volume 173 of ENTCS, pages 177-201. Elsevier, 2007.

32. A. Kurz, A. Palmigiano, and Y. Venema. Coalgebra and logic: A brief overview. Journal of Logic and Computation, 20:985-990, 2010.

33. K.G. Larsen and A. Skou. Bisimulation through probabilistic testing. Information and Computation, 94:1-28, 1991.

34. K.G. Larsen and A. Skou. Compositional verification of probabilistic processes. In Proc. of the 3rd Int. Conf. on Concurrency Theory (CONCUR 1992), volume 630 of LNCS, pages 456-471. Springer, 1992.

35. A. Parma and R. Segala. Logical characterizations of bisimulations for discrete probabilistic systems. In Proc. of the 10th Int. Conf. on Foundations of Software Science and Computation Structures (FOSSACS 2007), volume 4423 of LNCS, pages 287-301. Springer, 2007.

36. A. Philippou, I. Lee, and O. Sokolsky. Weak bisimulation for probabilistic systems. In Proc. of the 11th Int. Conf. on Concurrency Theory (CONCUR 2000), volume 1877 of LNCS, pages 334-349. Springer, 2000.

37. M.O. Rabin. Probabilistic automata. Information and Control, 6:230-245, 1963.

38. R. Segala. Modeling and Verification of Randomized Distributed Real-Time Systems. PhD Thesis, 1995.

39. R. Segala and N.A. Lynch. Probabilistic simulations for probabilistic processes. In Proc. of the 5th Int. Conf. on Concurrency Theory (CONCUR 1994), volume 836 of LNCS, pages 481-496. Springer, 1994.

40. R. Segala and A. Turrini. Comparative analysis of bisimulation relations on alternating and nonalternating probabilistic models. In Proc. of the 2 nd Int. Conf. on the Quantitative Evaluation of Systems (QEST 2005), pages 44-53. IEEE-CS Press, 2005.

41. A. Sokolova and E.P. de Vink. Probabilistic automata: System types, parallel composition and comparison. In Validation of Stochastic Systems, volume 2925 of LNCS, pages 1-43. Springer, 2004.

42. L. Song, L. Zhang, J.C. Godskesen, and F. Nielson. Bisimulations meet PCTL equivalences for probabilistic automata. Logical Methods in Computer Science, 9(2:7):1-34, 2013. 
43. M. Tracol, J. Desharnais, and A. Zhioua. Computing distances between probabilistic automata. In Proc. of the 9th Int. Workshop on Quantitative Aspects of Programming Languages (QAPL 2011), volume 57 of EPTCS, pages 148-162, 2011.

44. M.Y. Vardi. Automatic verification of probabilistic concurrent finite-state programs. In Proc. of the 26th IEEE Symp. on Foundations of Computer Science (FOCS 1985), pages 327-338. IEEE-CS Press, 1985.

45. E.P. de Vink and J.J.M.M. Rutten. Bisimulation for probabilistic transition systems: A coalgebraic approach. Theoretical Computer Science, 221:271-293, 1999.

46. Wang Yi and K.G. Larsen. Testing probabilistic and nondeterministic processes. In Proc. of the 12th Int. Symp. on Protocol Specification, Testing and Verification (PSTV 1992), pages 47-61. North-Holland, 1992. 